\title{
Discontinuity Formula and Sato's Conjecture
}

\author{
by \\ Takahiro KAWAI*)12) and Henry P. STAPP**)3)
}

\begin{abstract}
The $S$-matrix discontinuity formulas are used to show that in neighborhoods of many physical points the $S$-matrix is a holonomic microfunction.

In this paper we shall use the microlocal form of the $S$-matrix discontinuity formula to show that the $S$-matrix is a holonomic microfunction near many physical points.

A microfunction is holonomic only if it satisfies a holonomic system of micro-differential equations.***) The holonomicity of the $S$-matrix has been conjectured by Sato [1]. This property, if true in general, would mean that the $S$-matrix has, in a well-defined sense, the simplest possible type of singularity structure, and that this structure is amenable to study within the framework of the theory of holonomic microfunctions (SatoKawai-Kashiwara [2]. ${ }^{\dagger}$ )

According to the basic microanalyticity postulate the singularities of the $S$-matrix at physical points are associated with Landau diagrams. Consequently they can be classified in accordance with the complexity of these diagrams. Near singularities of the simplest class the holonomicity of the $S$-matrix follows directly from the microanalyticity postulate

Received September 8, 1975.

* Research Institute for Mathematical Sciences, Kyoto University, Kyoto, Japan and Department of Mathematics, University of California, Berkeey, California 94720.

** Lawrence Berkeley Laboratory, University of California, Berkeley, California 94720.

1) Supported by Miller Institute for Basic Research in Science.

2) Participating Guest: Lawrence Berkeley Laboratory.

s) Supported by the U. S. Energy Research and Development Administration.

*** In Sato-Kawai-Kashiwara [2] and Kawai-Stapp [3] a "holonomic system" is called a "maximally overdetermined system" and a "micro-differential equation (operator, resp.)" is called a "pseudo-differential equation (operator, resp.)". Here we use the new terminology proposed by Sato.

+ Hereafter we refer to this article as S-K-K [2] for brevity.
\end{abstract}


and the discontinuity formulas. To treat more complicated cases we introduce a complexified version of the microanalyticity postulate. This complexified version asserts that the singularities of the $S$-matrix near a physical point are confined to the local complexifications of the positive- $\alpha$ Landau surfaces, and enjoys there a certain boundedness property. Using this assumption we shall extend the analysis to a much broader class of singular points and show that the $S$-matrix near these points again satisfies a holonomic system of micro-differential equations whose characteristic variety is the union of the local complexifications of the positive$\alpha$ Landau surfaces, as demanded by our interpretation of the conjecture of Sato.

The method of proof suggests that an inductive procedure might yield the same result near all physical points but we have not obtained this result. In fact, our analysis covers only cases where the relevant Landau diagrams have at most two lines connecting any pair of vertices. Thus three-particle threshold points are excluded, along with all singularities that are locally related to singularities at three-particle threshold points.

In $\S 0$ we recall the definition of Landau varieties and related notions needed later. In $\S 1$ we write down the microlocal form of the discontinuity formula, and in $\S 2$ we give a detailed study of the $S$-matrix singularity structure under the assumption that the relevant Landau diagrams are simple (i.e., that at most one line joins any two vertices.) In $\S 3$ we discuss the case where the relevant Landau diagrams contain double internal lines. Singularities associated with Landau diagrams having more than two lines joining a pair of vertices are not considered in this work. In $\S 4$ we examine points where the simple triangle diagram singularity surface meets the associated normal threshold singularity surface, and in $\S 5$ we summarize our conclusions.

Some of the results of this paper have been announced in KawaiStapp [3]. The general procedure is to proceed in stages and investigate at each stage the singularities of the $S$-matrix that arise from the interplay of the singularities of the $S$-matrix proved at an earlier stage to be controlled by a holonomic system with singularities associated with the explicit mass-shell delta functions, which are also known to be controlled by holonomic systems. 


\section{§ 0. Preliminaries_-Space-time Landau equations, \\ Landau variety in the cotangent bundle, and related notions and results}

In this section we give the definition of the Landau equations, and also some related definitions that will be needed in the later sections. We also state and prove the structure theorem for bubble-diagram functions so that the significance of the space-time Landau equations becomes clearer.

Throughout this article it is assumed that the masses of the particles are strictly positive and have no accumulation point. Furthermore, it is assumed, for simplicity, that all the relevant particles are spinless. If $k$ is a four-vector, $k=\left(k_{0}, k_{1}, k_{2}, k_{3}\right)=\left(k_{0}, \vec{k}\right)$, then $k^{2}$ means $k_{0}{ }^{2}-k_{1}{ }^{2}-k_{2}{ }^{2}-k_{3}{ }^{2}$.

\section{Definition 0.1.}

A Landau diagram $D$ is a graph consisting of external lines $L_{r}^{e}$ $(r=1, \cdots, n)$, internal lines $L_{l}(l=1, \cdots, N)$ and vertices $V_{j}\left(j=1, \cdots, n^{\prime}\right)$. Each line is oriented and the orientation is denoted by an arrow $\rightarrow$. The structure of $D$ is determined by a set of incidence numbers $[j: r]$ and $[j: l]$ defined as follows (cf. Nakanishi [32] p. 14):

$[j: r]$ is $-1,+1$, or zero according to whether the line $L_{r}^{e}$ begins on $V_{j}$, terminates on $V_{j}$, or neither begins nor terminates on $V_{j}$. The numbers $[j: l]$ are defined in the analogous way. For each internal line $L_{l}$ there is a unique index $j^{-}(l)$ that satisfies $\left[j^{-}(l): l\right]=-1$ and a unique index $j^{+}(l)$ that satisfies $\left[j^{+}(l): l\right]=+1$. Thus the internal line $L_{l}$ begins at $V_{j-(l)}$ and terminates at $V_{j+(l)}$. For each external line $L_{r}^{e}$ there is a unique $j(r)$ such that $[j(r): r] \equiv \varepsilon_{r} \neq 0$. If $\varepsilon_{r}=+1$, then $L_{r}^{e}$ is called an initial line, if $\varepsilon_{r}=-1$ then $L_{r}^{e}$ is called a final line. Each internal line $L_{l}$ is associated with a stable particle the mass of which is a positive scalar $m_{l}>0$. Each external line $L_{r}^{e}$ is associated with a stable particle the mass of which is a positive scalar $\mu_{r}>0$.

\section{Definition 0.2.}

If for each pair of vertices $V_{j_{1}}$ and $V_{j_{2}}$ of a Landau diagram $D$ there exists at most one internal line $L_{l}$ that connects $V_{j_{1}}$ and $V_{j_{2}}$ (i.e., such 
that $\left[j_{1}: l\right] \neq 0$ and $\left.\left[j_{2}: l\right] \neq 0\right)$, then $D$ is called a simple diagram.

All diagrams $D$ used in this work are partially ordered.

\section{Definition 0.3.}

A Landau diagram $D$ is said to be partially ordered if and only if there is a partial ordering $>$ of the vertices $V_{j}$ of $D$ so that $V_{j^{+}(l)}>V_{j^{-}(l)}$ holds for every $l$. A partially ordered diagram $D$ can be drawn with $V_{j^{+}(l)}$ standing to the right of $V_{j^{-}(l)}$ for every $l$. Our diagrams $D$ will always be drawn in this way.

A flow line is a path in a Landau diagram that runs always from left to right, and cannot be lengthened.

\section{Definition 0.4.}

A signed Landau diagram $D$ is a Landau diagram $D$ such that each internal line $L_{l}$ of $D$ carries a $\operatorname{sign} \sigma_{l}$. This sign $\sigma_{l}$ is independent of the orientation of $L_{l}$.

\section{Definition 0.5.}

A set $\left(p_{1}, \cdots, p_{n} ; u_{1}, \cdots, u_{n}\right) \equiv(p ; u)$ consisting of $n$ real four-vectors $p_{r}$ and $n$ real four-vectors $u_{r}$ is said to be a solution of the Landau equations associated with the signed Landau diagram $D$ if and only if there are sets of real four-vectors $k_{l}(l=1, \cdots, N)$ and $v_{j}\left(j=1, \cdots, n^{\prime}\right)$ and real scalars $\alpha_{l}(l=1, \cdots, N)$ and $\beta_{r}(r=1, \cdots, n)$ such that the follow ing equations are satisfied:

$$
\left\{\begin{array}{lll}
\sum_{r=1}^{n}[j: r] p_{r}+\sum_{l=1}^{N}[j: l] k_{l}=0 & j=1, \cdots, n^{\prime} & (0.1 \mathrm{a}) \\
p_{r}{ }^{2}=\mu_{r}{ }^{2}, p_{r, 0}>0 & r=1, \cdots, n & (0.1 \mathrm{~b}) \\
k_{l}{ }^{2}=m_{l}{ }^{2}, k_{l, 0}>0 & l=1, \cdots, N & (0.1 \mathrm{c}) \\
\sum_{j=1}^{n}[j: l] v_{j}=\alpha_{l} k_{l} & l=1, \cdots, N \\
u_{r}=-\varepsilon_{r}\left(v_{j(r)}-\beta_{r} p_{r}\right) & r=1, \cdots, n \\
\sigma_{l} \alpha_{l} \geqq 0 & l=1, \cdots, N & (0.1 \mathrm{~d})
\end{array}\right.
$$




\section{Definition 0.6.}

The set of all solutions $(p ; u)$ of the Landau equations associated with $D$ is denoted by $\mathcal{L}(D)$.

If $D$ is unsigned then Definitions 0.5. and 0.6. still hold, except that the sign conditions $(0.1 \mathrm{f})$ are dropped from the Landau equations (0.1).

\section{Definition 0.7.}

If every internal line $L_{l}$ of $D$ carries a positive sign $\sigma_{l}=+1$, then $D$ is called a positive- $\alpha$ diagram. A corresponding solution of the Landau equations is called a positive- $\alpha$ solution. The symbol $D^{+}$represents a diagram that is the same as $D$ except that every sign $\sigma_{l}$ is positive.

Similarly we define $\mathcal{L}_{0}\left(D^{+}\right)$as follows:

\section{Definition 0.7.1.}

$\mathcal{L}_{0}\left(D^{+}\right)=\mathcal{L}\left(D^{+}\right)-\{(p ; u) ;(p ; u)$ is a solution of the Landau equations associated with $D^{+}$in which some $\left.\alpha_{l}=0\right\}$.

An important property of $\mathcal{L}_{0}\left(D^{+}\right)$is the following:

\section{Theorem 0. 0 .}

Suppose $D$ is an unsigned diagram and $\left(p_{0} ; u_{0}\right)$ lies on $\mathcal{L}_{0}\left(D^{+}\right)$. Then every solution $\left(p_{0} ; u_{0}\right)$ of the Landau equations associated with $D$ is a strictly positive- $\alpha$ solution with all $\alpha_{l}>0$.

Proof.

Suppose $\left(p_{0} ; u_{0}\right)$ were a solution corresponding to $D$ with some $\alpha_{l} \leqq 0$. A result of Pham [4] says that the corresponding $k_{l}$ 's are fixed by the mass-shell constraints and energy-momentum conservation equations alone, since $p$ lies in positive- $\alpha$ Landau surface. (See also Stapp [5] I. 19) Therefore each vector $k_{l}$ must coincide with the vector $k_{l}$ of the corresponding positive- $\alpha$ solution. Then the linearity of the Landau equation with respect to $(u, v, \alpha, \beta)$ with fixed $p_{r}$ and $k_{l}$ ensures that we can 
find some solution $(p ; u)$ with all $\alpha_{l} \geqq 0$ and some $\alpha_{l}=0$ of the Landau equation associated with $D$. This contradicts the assumption that $\left(p_{0} ; u_{0}\right)$ lies in $\mathcal{L}_{0}\left(D^{+}\right)$.

Q.E.D.

\section{Corollary.}

Suppose that $D$ is unsigned and that $(p ; u)$ belongs to $\mathcal{L}_{0}\left(D^{+}\right)$. Assume, furthermore, that no pair of four vector $\left(p_{r}, p_{r}{ }^{\prime}\right)$ with $r \neq r^{\prime}$ and $\varepsilon_{r}=\varepsilon_{r}^{\prime}$ are parallel. Then no solution $\left(p^{\prime} ; u^{\prime}\right)=(p ; 0)$ satisfies the Landau equation associated with $D$.

Proof.

The above theorem implies that the $u=0$ solution must be a strictly positive- $\alpha$ solution. However, under the nonparallelness condition on the $p_{r}$ there is no $u=0$ solution with all $\alpha_{l}>0$. This completes the proof of the corollary.

\section{Definition 0.8.}

A set $\left(p_{1}, \cdots, p_{n} ; u_{1}, \cdots, u_{n}\right) \equiv(p ; u)$ consisting of $n$ complex fourvectors $p_{r}$ and $n$ complex four-vectors $u_{r}$ is said to be a complex solution of the Landau equations associated with the Landau diagram $D$ if and only if there are sets of complex four-vectors $k_{l}$ and $v_{j}$ and complex scalars $\alpha_{l}$ and $\beta_{r}$ such that the complexified equations $(0.1 \mathrm{a}-\mathrm{e})$ are satisfied.

\section{Definition 0.9.}

The set of all complex solutions of the Landau equations associated with $D$ is denoted $\mathcal{L}^{c}(D)$. If there is no fear of confusions we omit the superscript $\mathbb{C}$.

By virtue of these definitions the sets $\mathcal{L}(D)$ and $\mathcal{L}^{c}(D)$, resp., are subvarieties of $S^{*} \boldsymbol{R}^{4 n}$ and $P^{*} \mathbb{C}^{4 n}$, resp.*) Here we have identified the cotangent vector $\operatorname{grad}_{k} k^{2}$ with $k$ itself by making use of the Minkowsky

* For a real analytic manifold $M$, we denote by $S^{*} M$ the cotangential spherical bundle, i.e., $\left(T^{*} M-M\right) / \boldsymbol{R}^{+}$. For a complex manifold $X$, we denote by $P^{*} X$ the cotangential projective bundle, i.e. $\left(T^{*} X-X\right) / C^{*}$. 
metric $k^{2}=k_{0}{ }^{2}-k_{1}{ }^{2}-k_{2}{ }^{2}-k_{3}{ }^{2}$. Furthermore, under the convention (0.2) ((0.3), resp.) specified below, the set $\mathcal{L}(D)$ ( $\mathcal{L}^{C}(D)$, resp.) is a subvariety of $S^{*} \mathscr{M}\left(P^{*} \mathscr{M}^{c}\right.$, resp.), where $\mathcal{M}$ is the mass-shell manifold $\mathscr{M}=\left\{p \in \boldsymbol{R}^{4 n} ; p_{r}{ }^{2}=\mu_{r}^{2} r=1, \cdots, n\right\}$ (and $\mathscr{M}^{\boldsymbol{c}}$ is its complexification i.e., $\mathscr{M}^{\boldsymbol{C}}=\left\{p \in \mathbb{C}^{4 n} ; p_{r}{ }^{2}=\mu_{r}{ }^{2} r=1, \cdots, n\right\}$. The conventions are:

(0.2) Two solutions $(p ; u)$ and $\left(p^{\prime} ; u^{\prime}\right)$ of the Landau equations are considered to be the same point if and only if both $p_{r}=p_{r}{ }^{\prime}$ $(r=1, \cdots, n)$ and $u_{r}-u_{r}^{\prime}=\gamma_{r} p_{r}(r=1, \cdots, \mathrm{n})$ hold for some real scalars $\gamma_{r}(r=1, \cdots, n)$.

(0.3) The same rule as in (0.2) except that $\gamma_{r}$ is allowed to be complex.

We may introduce another convention $\left(0.2^{\prime}\right)\left(\left(0.3^{\prime}\right)\right.$, resp.) so that $\mathcal{L}(D)\left(\mathcal{L}^{\boldsymbol{c}}(D)\right.$, resp.) defines a subvariety of $S^{*} \mathscr{M}_{r}\left(P^{*} \mathscr{M}_{r}{ }^{c}\right.$, resp.), where $\mathscr{M}_{r}$ is the restricted mass-shell variety $\mathscr{M}_{r}=\left\{p \in \mathbb{R}^{4 n} ; \sum_{r=1}^{n} \varepsilon_{r} p_{r}=0\right.$ and $p_{r}{ }^{2}=\mu_{r}{ }^{2}$ for $\left.r=1, \cdots, n\right\}$ and $\mathscr{M}_{r}{ }^{C}$ is its complexification i.e., $\mathscr{M}_{r}{ }^{C}$ $=\left\{p \in C^{4 n} ; \sum_{r=1}^{n} \varepsilon_{r} p_{r}=0\right.$ and $p_{r}^{2}=\mu_{r}^{2}$ for $\left.\left.r=1, \cdots, n\right\} . *\right)$

$\left(0.2^{\prime}\right)$ Two sets of four-vectors $(p ; u)$ and $\left(p^{\prime} ; u^{\prime}\right)$ are considered to be equivalent if and only if $p_{r}=p_{r}{ }^{\prime} \quad(r=1, \cdots, n)$ and $u_{r}-u_{r}{ }^{\prime}$ $=\gamma_{r} p_{r}+\varepsilon_{r} a(r=1, \cdots, n)$ hold for some real scalars $\gamma_{r}$ and some real four-vector $a$.

$\left(0.3^{\prime}\right)$ The same rule as in $\left(0.2^{\prime}\right)$ except that $\gamma_{r}$ and $a$ are allowed to be complex.

It is clear that these conventions are consistent with Landau equations (0.1). Hereafter, we shall often employ these conventions without explicitly citing them. Note that these conventions are the natural counterpart of the fact that the $S$-matrix $S(p)$ is a well-defined hyperfunction on $\mathcal{M}$ and the scattering amplitude $s(p)$ is a well-defined

* In order to avoid the singular point of $\mathcal{M}_{r}$ and $M_{r} C$, we consider the points where not all the $p_{r}$ 's are parallel. 
hyperfunction on $\mathscr{M}_{r}$ except for the singular points of $\mathscr{M}_{r}$. (See (1.2.1) of S-K-K [2] Chapter I. See also Lemma 0.2 at the end of this subsection.) Note also that $\hat{u}_{r} \equiv-u_{r} \varepsilon_{r}$ where $u_{r}$ is defined by $(0.1 \mathrm{e})$, is the set of vectors to points on the straight space-time trajectory line that passes through $v_{j(r)}$ and is parallel to $p_{r}$. This set of points is called the trajectory of the line $L_{r}^{e}$.

Note finally that we sometimes regard $\mathcal{L}(D)$ as a subvariety of the pure imaginary bundle $\sqrt{-1} S^{*} \mathcal{M}_{r}$ or $\sqrt{-1} S^{*} \mathcal{M}$ by the isomorphism $(p ; u) \widetilde{\rightarrow}(p ; \sqrt{-1} u)$. This is because the sheaf of microfunctions is defined on the pure imaginary bundle.

In connection with this geometric meaning of $(0.1 \mathrm{e})$, one can rewrite the equation (0.1) in the following form, which is used in Kashiwara-Kawai-Stapp [6].

\section{Definition 0.10 (Another form of the Landau equations)}

A set $(p ; u)$ consisting of $n$ real four-vectors $p_{r}$ and $n$ real fourvectors $u_{r}$ is said to be a solution of the Landau equations associated with the signed Landau diagram $D$ if and only if there exist real scalars $\beta_{l,+}$ and $\beta_{l,-}(l=1, \cdots, N)$ and $\beta_{r}(r=1, \cdots, n)$ and real four-vectors $k_{l}$ and $\widehat{\iota}_{l}(l=1, \cdots, N)$ and $v_{j}\left(j=1, \cdots, n^{\prime}\right)$ which satisfy the following:

$$
\begin{cases}\sum_{r=1}^{n}[j: r] p_{r}+\sum_{l=1}^{N}[j: l] k_{l}=0 & j=1, \cdots, n^{\prime} \\ p_{r}{ }^{2}=\mu_{r}{ }^{2}, \quad p_{r, 0}>0 & r=1, \cdots, n \\ k_{l}{ }^{2}=m_{l}{ }^{2}, \quad k_{l, 0}>0 & l=1, \cdots, N \\ \hat{u}_{l}=v_{j^{+}(l)}-\beta_{l,+} k_{l} & \\ =v_{j-(l)}-\beta_{l,-} k_{l} & l=1, \cdots, N \\ \hat{u}_{r} \equiv-\iota_{r} \varepsilon_{r}=v_{j(r)}-\beta_{r} p_{r} & r=1, \cdots, n \\ \sigma_{l}\left(\beta_{l,+}-\beta_{l,-}\right) \geqq 0 \text { for every line } l\end{cases}
$$

It is clear that the equations (0.4) are equivalent to the equations (0.1). In $(0.4 \mathrm{~d}), \hat{u}_{\imath}$ enjoys the same geometric interpretation as $\hat{u}_{r}$. Because of this symmetry of the quantities associated with the internal 
and external lines, the Landau equations given in the form of (0.4) are often more convenient and appeal more to intuition than the equation given in the form (0.1).

A solution to $(0.4)$ can be interpreted as a space-time diagram $\bar{D}$ that has the topological structure of $D$ with each space-time trajectory parallel or anti-parallel to the corresponding vector $k_{l}$ according to whether $\sigma_{l}=+$ or - .

We have introduced the notion of the complexified Landau variety $\mathcal{L}^{\boldsymbol{C}}(D)$ as the set of all complex solutions of the equation (0.1). Sometimes it is more convenient to consider the "local" complexification of $\mathcal{L}\left(D^{+}\right)$, in order to extend the "positive- $\alpha$ conditions" into the complex domain. For this purpose, we introduce the notion of the local complexification $\mathcal{L}^{\boldsymbol{C}}\left(D^{+} ; \omega\right)$ of $\mathcal{L}\left(D^{+}\right)$over an open set $\omega$.

First we define the connected component of $\mathcal{L}^{C}(D)$ relative to an open set in $(p ; u)$-space.

\section{Definition 0.11.}

Let $\omega$ be an open set in the complexification of $\sqrt{-1} S^{*} \mathcal{M}_{r}$. A connected component of $\mathcal{L}^{\boldsymbol{c}}(D)$ relative to $\omega$ is by definition a part $C$ of the variety defined by $(0.1)$ in complex $(p, u, k, v, \alpha, \beta)$-space that satisfies the following condition:

(0.5) $C$ lies over $\omega$ and is connected in $(p, u, k, v, \alpha, \beta)$-space.

\section{Definition 0.12.}

Let $\left(p_{0} ; u_{0}\right)$ be a point on $\mathcal{L}\left(D^{+}\right)$, and let $\omega$ be a neighborhood of $\left(p_{0} ; u_{0}\right)$. Then $\mathcal{L}^{c}\left(D^{+} ; \omega\right)$ is the projection on complex $(p ; u)$-space of the union of all the connected components of $\mathcal{L}^{c}(D)$ over $\omega$ that contain a strictly positive- $\alpha$ solution.

The solutions of the Landau equations discussed above are points in the cotangent bundle. The usual solutions of the Landau equations arise by projecting the nontrivial solutions of the Landau equations defined above into $p$ space. (A nontrivial solution has by definition some 
$\left.\alpha_{l}=0\right)$. Thus the usual solutions of the Landau equation are a set in $\mathscr{M}$ (or $\mathscr{M}_{r}$ or its complexification). This set will be called the Landau "surface," though it is not necessarily of codimension1. We denote it by $L(D)$, and its complexification by $L^{\boldsymbol{C}}(D)$.

An important subset of $L\left(D^{+}\right)$is the surface $L_{0}\left(D^{+}\right)$defined as follows:

\section{Definition 0.13.}

The restricted positive- $\alpha$ Landau surface $L_{0}\left(D^{+}\right)$is defined by $L_{0}\left(D^{+}\right) \equiv L\left(D^{+}\right)-\{p$; there is some nontrivial solution $(p ; u)$ with some $\alpha_{l}=0$ of the Landau equations associated with $\left.D^{+}\right\}$.

The restricted positive- $\alpha$ Landau surfaces enjoy several nice properties, that we shall use in $\S 2$. (See Chandler-Stapp [7].)

The set $\mathcal{L}^{+}$is the union of the sets $\mathcal{L}\left(D^{+}\right)$. Its importance arises from its close connection to the singularity spectrum of the $S$-matrix (See Chandler-Stapp [7], Iagolnitzer-Stapp [8], Pham [9], Sato [10], Kawai-Stapp [11] and Iagolnitzer [12]). There are, however, some exceptional points where the Landau equations fail to adequately limit the singularity spectrum. To deal with these points a modified system of equations has been proposed by Kashiwara-Kawai-Stapp [6]. However, that approach has not yet been sufficiently developed, so we shall in the present article merely identify the exceptional points, and exclude these interesting but troublesome points from our investigation.

The first kind of exceptional points are the so-called $\mathscr{M}_{0}$-points.

\section{Definition 0.14.}

A point $p=\left(p_{1}, \cdots, p_{n}\right) \in \mathcal{M}$ is called an $\mathscr{M}_{0}$-point if and only if $p_{r}$ and $p_{r}{ }^{\prime}$ are parallel for some $r \neq r^{\prime}$ with $\varepsilon_{r}=\varepsilon_{r}{ }^{\prime}$. An $\mathscr{M}_{0}$-point is a special case of the $u=0$ point defined below, in the sense that for any $\mathscr{M}_{0}$-point $p$ one can find a positive- $\alpha$ Landau diagram $D^{+}$for which $p$ is a $u=0$ point.

\section{Definition 0.15.}

A point $p=\left(p_{1}, \cdots, p_{n}\right)$ is said to be a $u=0$ point for a Landau 
diagram $D$ if and only if $(p ; u)=(p ; 0)$ is a nontrivial solution of the Landau equations associated with $D$.

The connection between $\mathcal{L}^{+}$and the singularity spectrum of the $S$-matrix is specified by the following microanalyticity property, which is equivalent to the macrocausality property of the $S$-matrix (IagolnitzerStapp [8], Iagolnitzer [12]).

\section{POSTUlate。}

The singularity spectrum of the S-matrix is confined to $\mathcal{L}^{+}=\bigcup_{D^{+}} \mathcal{L}\left(D^{+}\right)$, outside $\mathscr{M}_{0}$-points.

The set $\mathcal{L}^{+}$enjoys a finiteness property that guarantees that $\mathcal{L}^{+}$ is an analytic set. First recall the following theorem (Stapp [5]).

\section{Theorem 0.1 .}

$$
\mathcal{L}^{+}=\bigcup_{D^{+}} \mathcal{L}_{0}\left(D^{+}\right) \text {and } L^{+}=\bigcup_{D^{+}} L_{0}\left(D^{+}\right) \text {. }
$$

The finiteness property is this (Stapp [13]):

\section{Theorem 0.2 .}

Suppose that $R$ is a compact set in $M_{r}$. Then the number of $D^{+}$ such that $L_{0}\left(D^{+}\right) \cap R \neq \phi$ is finite.

This theorem guarantees that $\mathrm{L}^{+}$, hence $\mathcal{L}^{+}$, is a well-defined analytic set.

Manipulations with the unitarity and cluster decomposition properties of the $S$-matrix lead to the so-called bubble diagram functions, which are defined as follows:

\section{Definition 0.16.}

A bubble diagram $B$ is a diagram obtained by assigning a $\operatorname{sign} \sigma_{j}$ to each vertex $V_{j}$ of a partially ordered Landau diagram. (Pictorially, the vertex $V_{j}$ of a Landau diagram is represented as a point, whereas the corresponding signed vertex of a bubble diagram is represented as 
a bubble $b$ that contains the associated sign $\sigma_{b} \equiv \sigma_{j}$. See Fig. 1 below.)

$D:$

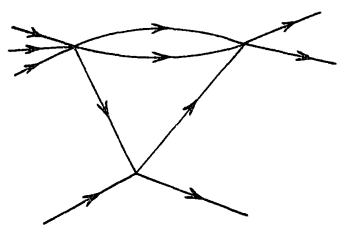

B:

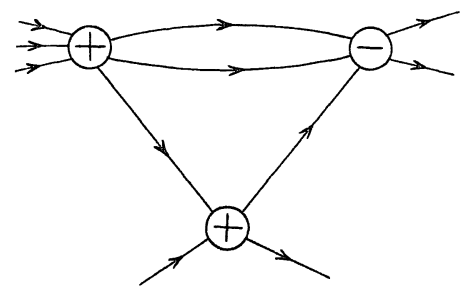

Fig. 1. A partially ordered Landau diagram $D$ and a bubble diagram $B$ constructed from it.

\section{Definition 0. 17.}

The incidence numbers $[b: r]$ and $[b: l]$ are defined in the same way as corresponding numbers $[j: r]$ and $[j: l]$, i.e., $[b: r]$ is $-1,+1$ or zero according to whether the line $L_{r}^{e}$ begins on $b$, terminates on $b$, or neither begins nor terminates on $b$, etc.

\section{Definition 0. 18.}

The bubble diagram function $F^{B}(p)$ is the function obtained from the bubble diagram $B$ applying the following procedures:

(0.9) Replace each plus bubble $b$ of $B$ by the connected part of the corresponding covariantly normalized $S$-matrix element $\left\langle p_{b}{ }^{\text {out }}|S| p_{b}{ }^{\text {in }}\right\rangle$, where $p_{b}{ }^{\text {out }}$ is the set of momentum vectors associated with the lines of $B$ that begin on bubble $b$ and $p_{b}{ }^{\text {in }}$ is the set of momentum vectors associated with the lines of $B$ that terminate on $b$.

(0.10) Replace each minus bubble $b$ of $B$ by minus the connected part of the complex conjugate of the matrix element $\left\langle p_{b}{ }^{\text {in }}|S| p_{b}{ }^{\text {out }}\right\rangle$ with $p_{b}{ }^{\text {in }}$ and $p_{b}{ }^{\text {out }}$ defined as above:

(0.11) Replace each internal line $L_{l}$ of $B$ by a factor $\sum_{l} 2 \pi \delta^{+}\left(k_{l}{ }^{2}-m_{l}{ }^{2}\right)$ $=\sum_{l} 2 \pi \delta\left(k_{l}{ }^{2}-m_{l}{ }^{2}\right) Y\left(k_{l, 0}\right)$, where $Y$ is the Heaviside function, and the sum is over particle-types $t$, and $m_{l}$ is the mass of particles of type $t$.

(0.12) Perform an integration $\frac{1}{(2 \pi)^{4}} \int d^{4} k_{l}$ over the momentum vector $k_{l}$ associated with each internal $L_{l}$ of $B$. 
(0.13) Divide by $n$ !, where $n$ is the number of independent symmetry operations of $B$. Here a symmetry operation means a transformation $\sigma=\left(\sigma_{1}, \sigma_{2}\right)$ of the indices $j \stackrel{\sigma_{1}}{\longrightarrow} j^{\prime}$ and $l \stackrel{\sigma_{2}}{\longrightarrow} l^{\prime}$ such that $[j: l]=\left[\sigma_{1}(j): \sigma_{2}(l)\right]$ and $[j: r]=\left[\sigma_{1}(j): r\right]$ hold for every $j$, $l$ and $r$.

\section{Remark.}

It is obvious that procedures $(0.11)$ and $(0.12)$ can be combined so that the integration procedure is performed on mass-shell as follows:

(0.14) Substituting $\sqrt{\vec{k}_{l}{ }^{2}+m_{l}{ }^{2}}$ for $k_{l, 0}$ and performing an integration $\sum_{l} \frac{1}{16 \pi^{3}} \int \frac{d^{3} \vec{k}_{l}}{\sqrt{\vec{k}_{l}{ }^{2}+m_{l}{ }^{2}}}$ over each internal line $L_{l}$ of $B$.

\section{Definition 0.19.}

A Landau diagram $D$ is said to fit into a bubble diagram $B$ if and only if $D$ is a signed diagram that can be constructed by replacing each bubble $b$ of $B$ by a connected partially-ordered Landau diagram $D_{b}$, which may be simply a point. The initial (final, resp.) lines of the diagram $D_{b}$ that replaces bubble $b$ are to coincide in a one-to-one way with the lines of $B$ that terminate (begin, resp.) on that bubble. Each internal line of the diagram $D_{b}$ that replaces a bubble $b$ is to carry the sign $\sigma_{b}$ of that bubble. The remaining internal lines of $D^{\sigma}$, which are precisely the internal lines of the original bubble diagram, can carry any signs.

The set of $D$ that fits into a bubble diagram $B$ is denoted by $\bar{B}$.
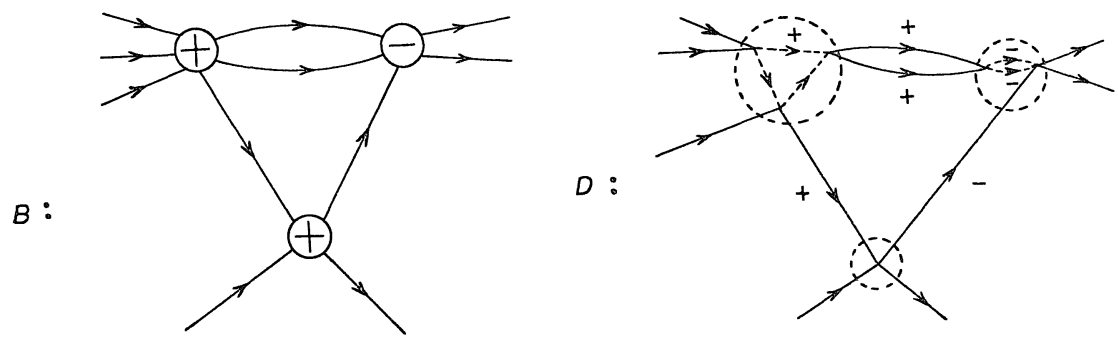

Fig. 2. An example of a Landau diagram $D$ that fits into bubble diagram $B$. 
Remark.

The lines of a bubble diagram $B$ are sometimes called explicit lines, to distinguish them from the lines of a $D \in \bar{B}$ that are internal lines of some $D_{b}$.

In Definition 0.18 we inserted the connected part of $S$ or its complex conjugate $S^{\dagger}$ multiplied by $(-1)$ for each vertex of $D$. Sometimes we use instead the function obtained by inserting the entire $S$-matrix $S(p)$ itself, or its complex conjugate $S^{\dagger}(p)$, for certain vertices. In such a case one uses a small box, with a plus or minus sign inside, instead of a small bubble. The connectedness required in Definition 0.19 for the diagram $D_{b}$ that replaces a bubble $b$ is not required for the diagram $D_{b}$ that replaces a box $b$.

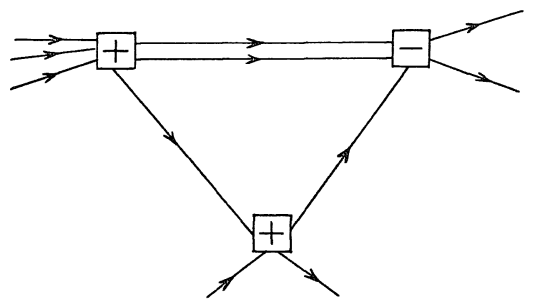

Fig. 3. An example of a diagram with boxes.

Since the (local) analytic properties of the function corresponding to a diagram with boxes can be immediately derived from those of the bubble diagram functions we discuss here only the analytic properties of the latter.

\section{Definition 0.20.}

A solution of the Landau equations associated with a bubble diagram function $F^{B}(p)$ is, by definition, a solution $(p ; u)$ of the Landau equation (0.1) corresponding to some $D$ that fits into $B$. If $(p ; u)=(p ; 0)$ is a solution with some $\alpha_{l} \neq 0$ of the Landau equation associated with some $D \in \bar{B}$, we say that $p$ is a $u=0$ point of $F^{B}(p)$.

Definition 0.20 is justified by the following structure theorem (Stapp [14], Iagolnitzer [15] and Kawai-Stapp [16]).

\section{Structure Theorem.}

If the singularity spectrums of the bubble diagram functions 
$F^{B_{2}}\left(p^{\prime}, p_{1}, \cdots, p_{s}\right)\left(p^{\prime}=\left(p_{s+1}, \cdots, p_{n^{\prime}}\right)\right)$ and $F^{B_{2}}\left(p_{1}, \cdots, p_{s}, p^{\prime \prime}\right)\left(p^{\prime \prime}=\left(p_{s+1}, \cdots\right.\right.$, $\left.\left.p_{n^{\prime}}\right)\right)$ are confined to solutions of the associated Landau equations, except possibly at $u=0$ points, then the bubble diagram function $F^{B}\left(p^{\prime}\right.$, $\left.p^{\prime \prime}\right)$ corresponding to the bubble diagram $B$ obtained by joining $B_{1}$ and $B_{2}$ with respect to $p_{1}, \cdots, p_{s}$ has the same property.

The usual formulation of the structure theorem follows from a repeated application of the above theorem, starting from bubble diagrams $B_{1}$ and $B_{2}$ consisting of single bubbles. For these simplest diagrams the micro-analyticity postulate for the $S$-matrix validates the assumption of the theorem outside $\mathscr{M}_{0}$-points. In fact, $\mathscr{M}_{0}$-points are the only $u=0$ points for these simplest diagrams. Moreover, the macro-causality property discussed by Iagolnitzer-Stapp [8] does not give any conditions on the singularity spectrum of the $S$-matrix at the $\mathscr{M}_{0}$-points. Accordingly, the $u=0$ points are excluded in the hypothesis of the structure theorem, and they are also excluded from the conclusions. (Progress on the extension of the Structure Theorem to $u=0$ points has been made by Kashiwara-Kawai-Stapp [6], but we shall not describe those results here).

In view of the importance of the structure theorem, we give here a simple proof of the theorem based on the theory of microfunctions (S-K-K [2] Chapter I). An analogous proof based on the theory of essential support of distributions developed by Bros-Iagolnitzer [17] has been given by Iagolnitzer [15]. The proof given here will both establish our terminology, and also prepare the way for similar arguments to be given in subsequent sections.

\section{Proof of the Structure Theorem.}

Let $\mathscr{M}_{1}, \mathscr{M}_{2}$, and $\mathscr{M}$ be the mass-shells on which $F^{B_{1}}\left(p^{\prime}, p_{1}, \cdots, p_{s}\right)$, $F^{B_{2}}\left(p_{1}, \cdots, p_{s}, p^{\prime \prime}\right)$ and $F^{B}\left(p^{\prime}, p^{\prime \prime}\right)$ are defined, respectively. Denote by $\widetilde{\mathscr{M}}$ the manifold defined by $\left\{\left(p^{\prime}, p_{1}, \cdots, p_{s}, p^{\prime \prime}\right) ;\left(p^{\prime}, p_{1}, \cdots, p_{s}\right) \in \mathscr{M}_{1}\right.$ and $\left.\left(p_{1}, \cdots, p_{s}, p^{\prime \prime}\right) \in \mathscr{M}_{2}\right\}$.

According to Definition $0.18, F^{B}\left(p^{\prime}, p^{\prime \prime}\right)$ is given by a sum of terms

$$
\begin{aligned}
\frac{1}{n !(2 \pi)^{3 s}} \int \cdots \int F^{B_{1}}\left(p^{\prime}, p_{1}, \cdots, p_{s}\right) \prod_{l=1}^{s} \delta\left(p_{l}{ }^{2}-\mu_{l}{ }^{2}\right) \times \\
\times Y\left(p_{l, 0}\right) F^{B_{2}}\left(p_{1}, \cdots, p_{s}, p^{\prime \prime}\right) d^{4} p_{1} \cdots d^{4} p_{s}
\end{aligned}
$$




$$
\begin{aligned}
& =\frac{1}{n !\left(16 \pi^{3}\right)^{s}} \int_{p_{l^{2}=\mu_{l}{ }^{2}}} \ldots \int_{F^{B_{1}}}\left(p^{\prime}, p_{1}, \cdots, p_{s}\right) F^{B_{2}}\left(p_{1}, \cdots, p_{s}, p^{\prime \prime}\right) \times \\
& \quad \times \prod_{l=1}^{s} \frac{1}{\sqrt{\vec{p}_{l}{ }^{2}+\mu_{l}{ }^{2}}} d^{3} \vec{p}_{1} \cdots d^{3} \vec{p}_{s} .
\end{aligned}
$$

We first show that the integrand of $(0.15)$ is a well-defined hyperfunction on $\widetilde{\mathscr{M}}^{\prime} \equiv\left\{\left(p^{\prime}, p_{1}, \cdots, p_{s}, p^{\prime \prime}\right) \in \widetilde{\mathscr{M}} ;\left(p^{\prime}, p^{\prime \prime}\right)\right.$ is not a $u=0$ point of $F^{B}$.

Theorem 2.4.1 of S-K-K [2] Chapter I asserts that the singularity spectrum of $F^{B_{1}}$ regarded as a hyperfunction on $\tilde{\mathcal{M}}^{\prime}$ is given by

(0. 16) $A_{1}=\left\{\left(p^{\prime}, p_{1}, \cdots, p_{s}, p^{\prime \prime} ; \sqrt{-1}\left(u^{\prime}, u_{1}, \cdots, u_{s}, u^{\prime \prime}\right)\right) \in \sqrt{-1} S^{*} \tilde{\mathscr{M}}^{\prime}\right.$;

$$
\left(p^{\prime}, p_{1}, \cdots, p_{s} ; \sqrt{-1}\left(u^{\prime}, u_{1}, \cdots, u_{s}\right)\right) \in \text { S.S. } F^{B_{1}} \subset \sqrt{-1} S^{*} \mathscr{M}_{1},
$$
and $\left.u^{\prime \prime}=0\right\}$.

Analogously the singularity spectrum of $F^{B_{2}}$ regarded as a hyperfunction on $\tilde{\mathscr{H}}^{\prime}$ is given by

$$
\begin{aligned}
& A_{2}=\left\{\left(p^{\prime}, p_{1}, \cdots, p_{s}, p^{\prime \prime} ; \sqrt{-1}\left(u^{\prime}, u_{1}, \cdots, u_{s}, u^{\prime \prime}\right)\right) \in \sqrt{-1} S^{*} \tilde{\mathscr{M}}^{\prime}\right. \\
& \left(p_{1}, \cdots, p_{s}, p^{\prime \prime} ; \sqrt{-1}\left(u_{1}, \cdots, u_{s}, u^{\prime \prime}\right)\right) \in \text { S.S. } F^{B_{2}} \subset \sqrt{-1} S^{*} \mathcal{M}_{2}, \\
& \text { and } \left.u^{\prime}=0\right\} \text {. }
\end{aligned}
$$

Since $\prod_{l=1}^{s} \frac{1}{\sqrt{\vec{p}_{l}{ }^{2}+\mu_{l}{ }^{2}}}$ is analytic, it does not give any effect on the singularity spectrum of the integrand of (0.15).

Corollary 2.4.2 of S-K-K [2] Chapter I asserts that $F^{B_{1}} F^{B_{2}}$ is a well-defined hyperfunction on $\tilde{\mathcal{H}}^{\prime}$ if $A_{1} \cap A_{2}{ }^{a}=\phi$.

Suppose there were some point in $A_{1} \cap A_{2}{ }^{a}$. This would imply the existence of a set of vectors $\left(p^{\prime}, p_{1}, \cdots, p_{s}, p^{\prime \prime} ; u_{1}, \cdots, u_{s}\right)$ such that:

(0.18) $\quad\left(p^{\prime}, p_{1}, \cdots, p_{s} ; \sqrt{-1}\left(0, u_{1}, \cdots, u_{s}\right)\right) \in \mathrm{S} . \mathrm{S} . F^{B_{1}} \subset \sqrt{-1} S^{*} \mathscr{M}_{1}$

and that

(0. 19) $\left(p_{1}, \cdots, p_{s}, p^{\prime \prime} ;-\sqrt{-1}\left(u_{1}, \cdots, u_{s}, 0\right)\right) \in$ S.S. $F^{B_{2}} \subset \sqrt{-1} S^{*} \mathscr{M}_{2}$.

The point $\left(p^{\prime}, p_{1}, \cdots, p_{s}, p^{\prime \prime}\right)$ satisfies

$$
\left(p^{\prime}, p_{1}, \cdots, p_{s}\right) \in \pi\left(\text { S.S. } F^{B_{1}}\right)=\operatorname{Supp} F^{B_{1}}
$$

and 


$$
\left(p_{1}, \cdots, p_{s}, p^{\prime \prime}\right) \in \pi\left(\text { S.S. } F^{B_{2}}\right)=\operatorname{Supp} F^{B_{2}} .
$$

For such a point the condition that $\left(p^{\prime}, p^{\prime \prime}\right)$ not be a $u=0$ point of $F^{B}$ implies that $\left(p^{\prime}, p_{1}, \cdots, p_{s}\right)$ is not a $u=0$ point of $F^{B_{1}}$. For from a $u=0$ solution of the Landau equations associated with $B_{1}$ one can construct a $u=0$ solution of the Landau equations associated with $B$ by placing at the origin all vertices of $B$ that are not vertices of $B_{1}$.

The condition that $\left(p^{\prime}, p_{1}, \cdots, p_{s}\right)$ not be a $u=0$ point for $B_{1}$, together with the hypothesis of the theorem implies that S.S. $F^{B_{1}}$ is confined to the solutions of the Landau equations associated with $B_{1}$. The same is true for S.S. $F^{B_{2}}$. Thus the set of vectors $\left(p^{\prime}, p_{1}, \cdots, p_{s} ; 0, u_{1}, \cdots, u_{s}\right)$ appearing in (0.18) is a solution of the Landau equations associated with $B_{1}$, and the set of vectors $\left(p_{1}, \cdots, p_{s}, p^{\prime \prime} ;-u_{1}, \cdots,-u_{s}, 0\right)$ appearing in (0.19) is a solution of the Landau equations associated with $B_{2}$. In particular, there exist four-vectors $v_{j(l)}$ and $\widetilde{v}_{j(l)}$ and constants $b_{l}$ and $\tilde{b}_{l}$ such that the equations

$$
-u_{l} \varepsilon_{l} \equiv \widehat{u}_{l}=v_{j(l)}-b_{l} p_{l}=\widetilde{v}_{j(l)}-\tilde{b}_{l} p_{l}
$$

hold for $l=1, \cdots, s$. The four-vector $v_{j(l)}$ gives the position of the external vertex of $B_{1}$ that is connected to line $l$, and $\widetilde{v}_{j(l)}$ gives the position of the external vertex of $B_{2}$ that is connected to line $l$. The above equation implies

$$
v_{j(l)}-\widetilde{v}_{j(l)}=\left(b_{l}-\tilde{b}_{l}\right) p_{l},
$$

which are the only Landau equations associated with $B$ that are not entailed by those for $B_{1}$ and $B_{2}$. Thus the set of vectors $\left(p^{\prime}, p_{1}, \cdots, p_{s}\right.$, $\left.p^{\prime \prime} ; 0, u_{1}, \cdots, u_{s}, 0\right)$ corresponds to a solution $\left(p^{\prime}, p^{\prime \prime} ; 0,0\right)$ of the Landau equations associated with $B$. But the existence of such a solution would mean that $\left(p^{\prime}, p^{\prime \prime}\right)$ is a $u=0$ point for diagram $B$, contrary to the condition on $\tilde{\mathscr{M}}^{\prime}$. Thus this condition on $\tilde{\mathscr{M}}^{\prime}$ implies that $A_{1} \cap A_{2}{ }^{a}=\phi$, and hence, by Corollary 2.4.2 of S-K-K [2] Chapter I that the integrand $F^{B_{1}} F^{B_{2}}$ of $(0.15)$ is well-defined as a hyperfunction on $\tilde{\mathscr{M}}^{\prime}$.

Corollary 2.4.2 of S-K-K [2] Chapter I may be used again to obtain information about S.S. $\left(F^{B_{1}} F^{B_{2}} \mid \widetilde{\mathscr{M}}^{\prime}\right)$. In particular, equations (0.16) and (0.17) imply that S.S. $\left(F^{B_{1}} F^{B_{2}} \mid{\widetilde{\mathfrak{M}^{\prime}}}^{\prime}\right)$ is contained in

(0.20) $\left\{\left(p^{\prime}, p_{1}, \cdots, p_{s}, p^{\prime \prime} ; \sqrt{-1}\left(\eta^{\prime}, \eta_{1}, \cdots, \eta_{s}, \eta^{\prime \prime}\right)\right) \in \sqrt{-1} S^{*} \widetilde{\mathscr{M}}^{\prime}\right.$; there 
exist non-negative real constants $c_{1}$ and $c_{2}$ with $c_{1}+c_{2}>0$ such that the following conditions $(0.21) \sim(0.25)$ hold .

(0.24) $\quad\left(p^{\prime}, p_{1}, \cdots, p_{s} ; \sqrt{-1}\left(u^{\prime}, u_{1}, \cdots, u_{s}\right)\right)$ is contained in S.S. $F^{B_{1}} \subset \sqrt{-1} S^{*} \mathscr{M}_{1}$.

$(0 \cdot 25) \quad\left(p_{1}, \cdots, p_{s}, p^{\prime \prime} ; \sqrt{-1}\left(\widetilde{u}_{1}, \cdots, \widetilde{u}_{s}, u^{\prime \prime}\right)\right)$ is contained in S.S. $F^{B_{2}} \subset \sqrt{-1} S^{*} \mathcal{M}_{2}$.

Note that $\operatorname{sing} \operatorname{supp} F^{B_{j}} \equiv \pi\left(\right.$ S.S. $\left.F^{B_{j}}\right)=\operatorname{Supp} F^{B_{j}}$ holds on $\mathscr{M}_{j}(j=1$, 2 , resp.) in our case (because of the conservation-law $\delta$-function factor in $F^{B_{j}}$ ). This implies that $F^{B_{1}} F^{B_{2}}$ is zero as a hyperfunction on $\tilde{\mathscr{M}}$ if either $\left(p^{\prime}, p_{1}, \cdots, p_{s}\right) \notin \operatorname{sing} \operatorname{supp} F^{B_{1}}$ or if $\left(p_{1}, \cdots, p_{s}, p^{\prime \prime}\right) \notin \operatorname{sing} \operatorname{supp} F^{B_{2}}$. Thus there is no contribution to S.S. $\left(F^{B_{1}} F^{B_{2}}\right)$ from the set where $F^{B_{1}}$ is analytic and $F^{B_{2}}$ is not analytic or from the set where $F^{B_{1}}$ is not analytic and $F^{B_{2}}$ is analytic.

Restricting $\left(p^{\prime}, p^{\prime \prime}\right)$ to $\tilde{\mathscr{M}^{\prime}}$ we may, using arguments given before, write

$$
\hat{u}_{l}=v_{j(l)}-b_{l} p_{l} \quad \text { and } \quad \widehat{\widehat{u}}_{l}=\widetilde{v}_{j(l)}-\widetilde{b}_{l} p_{l} \quad(l=1, \cdots, s) .
$$

Now we can apply Theorem 2.3.1 of S-K-K [2] Chapter I to the integral given by $(0.15)$. Because of the partial ordering condition on bubble diagrams the region of integration is compact, for $\left(p^{\prime}, p^{\prime \prime}\right)$ in compact sets, and hence the integration procedure is legitimate as an integration of a hyperfunction. Thus Theorem 2.3.1 of S-K-K [2] Chapter I applies to the integral given by (0.15), and it asserts that S.S. $F^{B}\left(p^{\prime}, p^{\prime \prime}\right)$ is contained in

(0.27) $\left\{\left(p^{\prime}, p^{\prime \prime} ; \sqrt{-1}\left(\eta^{\prime}, \eta^{\prime \prime}\right)\right) \in \sqrt{-1} S^{*} \mathcal{M}\right.$; there exists a set $\left(p_{1}, \cdots\right.$, $\left.p_{s}\right)$ such that $\left(p^{\prime}, p_{1}, \cdots, p_{s}, p^{\prime \prime} ; \sqrt{-1}\left(\eta^{\prime}, 0, \cdots, 0, \eta^{\prime \prime}\right)\right)$ is in the set defined in $(0.20)\}$.

Substituting (0.26) into (0.22) with $\eta_{l}=0$, i.e., into $c_{1} u_{l}+c_{2} \widetilde{u}_{l}=0$ $(l=1, \cdots, s)$, one obtains 


$$
c_{1} v_{j(l)}-c_{2} \widetilde{v}_{j(l)}=\left(c_{1} b_{l}-c_{2} \tilde{b}_{l}\right) p_{l}, \quad l=1, \cdots, s
$$

These are precisely the Landau equations associated with $B$ that are not already implied by the conditions demanded in (0.20). Since $c_{1}$ and $c_{2}$ are non-negative and $c_{1}+c_{2}>0$, the signs of the Landau constants $\alpha_{l}$ are preserved in the sense of $(0.1 \mathrm{f})$. That is, the signs of the $\alpha_{l}$ associated with lines of $B$ that are internal lines of the Landau diagram associated with $B_{1}$ or $B_{2}$ are preserved. But the signs of the $\alpha_{\imath}$ associated with the lines corresponding to $p_{1}, \cdots, p_{s}$ are not restricted.

This completes the proof of the theorem.

So far we stated and proved the structure theorem for bubble diagram functions. However, the bubble diagram function $F^{B}(p)$ contains an over-all $\delta$-function $\delta^{4}\left(\sum_{r} \varepsilon_{r} p_{r}\right)$. It is sometimes useful to have the structure theorem for the reduced bubble diagram function $f^{B}(p)$ $\left.=F^{B}(p) /(2 \pi)^{4} \delta^{4}\left(\sum_{r} \varepsilon_{r} p_{r}\right) *\right)$ defined on the reduced mass-shell manifold $\mathcal{M}_{r}$. For this purpose we prepare the following lemma, which has also its own interest.

\section{Lemma 0.2.}

Let $N$ be a real analytic submanifold of a real analytic manifold $M$. Let $\mu$ be a hyperfunction defined on $N$. Denote by $\delta_{N}$ the $\delta$ function supported by $N$ and define the hyperfunction $\nu$ by $\nu \equiv \mu \delta_{N}$. Then

$$
\text { S.S. } \nu=\rho^{-1}(\text { S.S. } \mu) \cup \sqrt{-1} S_{N} * M \text {. }
$$

Here $\rho$ is the canonical projection from $\sqrt{-1} S^{*} M \times \underset{M}{\times} N-\sqrt{-1} S_{N} * M$ to $\sqrt{-1} S^{*} N$.

Proof.

Corollary 2.4.2 of S-K-K [2] Chapter I claims that S.S. $\nu \subset$ $\rho^{-1}$ (S.S. $\left.\mu\right) \cup \sqrt{-1} S_{N} * M$. So, it suffices to show that

$$
\left(\rho^{-1}(\text { S.S. } \mu) \cup \sqrt{-1} S_{N} * M\right) \subset \text { S.S. } \nu \text {. }
$$

\footnotetext{
* If a function $M(p)$ is known to have the form $(2 \pi)^{4} \delta^{4}\left(\sum_{\tau} \varepsilon_{r} p_{r}\right) m(p)$ with some function $m(p)$, we sometimes use the notation $M(p) /(2 \pi)^{4} \delta^{4}\left(\sum_{r} \varepsilon_{r} p_{r}\right)$ to denote $m(p)$, though $m(p)$ is well-defined only on $\left\{p ; \sum_{r} \varepsilon_{r} p_{r}=0\right\}$.
} 


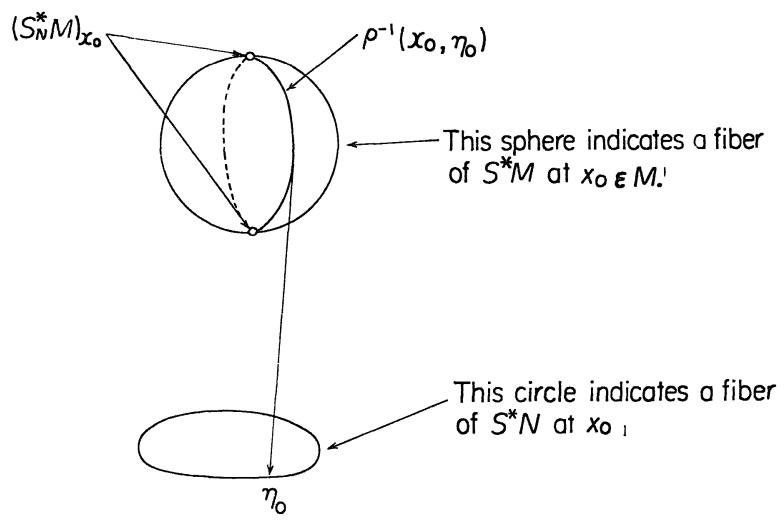

Fig. 5.

Since the statement is of local character, we fix a local coordinate system $x=\left(x_{1}, \cdots, x_{d} ; x_{d+1}, \cdots, x_{\eta}\right)=\left(x^{\prime} ; x^{\prime \prime}\right) \in M$ such that $N$ is given by $x^{\prime}=0$. We denote the corresponding cotangent vector by $\eta=\left(\eta^{\prime}, \eta^{\prime \prime}\right)$. Clearly $\nu(x)$ satisfies the (differential) equation

$$
x_{j} \nu=0 \quad(j=1, \cdots, d) .
$$

Therefore, a fundamental theorem on propagation of analyticity of solutions of linear micro-differential equations (Theorem 2.1.7 of S-K-K [2] Chapter III) tells us that the following two conditions are equivalent:

$$
\left(0, x^{\prime \prime} ; \sqrt{-1}\left(a, \eta^{\prime \prime}\right)\right) \in \text { S.S. } \nu \quad \text { for some } a \in \mathbb{R}^{d}
$$

$$
\left(0, x^{\prime \prime} ; \sqrt{-1}\left(a, \eta^{\prime \prime}\right)\right) \in \text { S.S. } \nu \quad \text { for any } a \in \mathbb{R}^{d}
$$

On the other hand, it is clear that

$$
\int \nu(x) d x^{\prime}=\mu\left(x^{\prime \prime}\right) \text {. }
$$

Therefore, by Theorem 2.3.1 of S-K-K [2] Chapter I $\left(0, x^{\prime \prime}\right.$; $\left.\sqrt{-1}\left(0, \eta^{\prime \prime}\right)\right)$ must be contained in S.S. $\nu$ if $\left(x^{\prime \prime} ; \sqrt{-1} \eta^{\prime \prime}\right)$ is contained in S.S. $\mu\left(x^{\prime \prime}\right)$. Then, taking into account the equivalence of (0.30) and (0.31), we conclude that $\rho^{-1}$ (S.S. $\left.\mu\right)$ is contained in S.S. $\nu$. It is clear that $\sqrt{-1} S_{N} * M \subset$ S.S. $\nu$, because Supp $\nu \subset N$. (See Proposition 2.1.3 of S-K-K [2] Chapter III.) Thus we have shown that

$$
\text { S.S. } \nu=\rho^{-1}(\text { S.S. } \mu) \cup \sqrt{-1} S_{N} * M \text {. }
$$

This completes the proof of the lemma. 
As we noticed earlier, the Landau equation (0.1) defines a subvariety of $S^{*} \mathcal{M}_{r}$ under the convention $\left(0.2^{\prime}\right)$. Then, applying Lemma 0.2 to our case, it is easy to see that Structure Theorem holds for reduced bubble diagram functions as it stands, if we consider the Landau equation to define a subvariety of $S^{*} \mathcal{M}_{r}$.

\section{$\S 1$. Microlocal form of the $S$-matrix discontinuity formula}

The presently existing derivations of the general $S$-matrix discontinuity formula rely on the assumption of "mixed- $\alpha$ cancellation." This assumption circumvents two problems. The first problem is the failure of the structure theorem to give any information at $u=0$ points. The second problem is the possibility that certain singularities associated with nonpositive- $\alpha$ diagrams might fail to cancel among themselves in the expected manner.

The problem with $u=0$ points poses a serious problem for the derivation of the discontinuity formula solely from the unitarity and macrocausality properties of the $S$-matrix. Even in the simplest case of the pole-factorization theorem certain of the occurring bubble diagram functions have open sets of $\iota=0$ points that would disrupt the proof were they not ruled out by the mixed- $\alpha$ cancellation assumption (See Iagolnitzer-Stapp [18]).

Examples indicate that these open sets of $\iota=0$ points are, in general, not actually singularities. They are allowed to be singular by the presently existing structure theorem, but they are in fact not actually singular. What is needed, therefore, is an improved version of the structure theorem that deals more effectively with these $u=0$ points and rules out the spurious open sets of singularities allowed by the present theorem. The recent work of Kashiwara-Kawai-Stapp [6] is an initial step toward the construction of a more adequate structure theorem.

After resolving this problem of $u=0$ points there will remain the problem of showing that the singularities associated with nonpositive- $\alpha$ diagrams cancel among themselves in the expected manner. In the work of Iagolnitzer-Stapp on the pole-factorization theorem it was shown that the necessary cancellations were entailed by an analyticity requirement 
that goes beyond microanalyticity. This requirement demands a limited amount of analyticity in a complex neighborhood of the physical region.

Arguments making full use of the results of Kashiwara-Kawai-Stapp, and employing a stronger analyticity assumption, have not yet been developed to give a proof of the general discontinuity formula not relying on the mixed- $\alpha$ cancellation assumption. However, the strong version of the mixed- $\alpha$ cancellation assumption needed for the presently existing derivations of the general formula is not fully justifiable, a priori. Thus we shall give here an alternative derivation that covers the cases used in the present work. This alternative derivation is based on the requirement of "separation of singularities." This requirement is more satisfactory than the mixed- $\alpha$ cancellation assumption, and provides a powerful new tool for the analysis of the $S$-matrix singularity structure.

The present work is based on the microlocal version of the discontinuity formula. To state this version we first recall the definitions of "contraction" and "contain."

\section{Definition 1. 1.}

A signed Landau diagram $D_{1}$ is said to contract to a signed diagram $D_{2}$ if and only if there is a one-to-one mapping of the internal lines of $D_{2}$ onto a subset $s$ of the internal lines of $D_{1}$ such that the contraction to points of all the internal lines of $D_{1}$, not in $s$ reduces $D_{1}$ to $D_{2}$ including signs. Each part of $D_{1}$ that reduces to a vertex of $D_{2}$ must be connected.

The internal lines of $D_{2}$ can be separated into subsets such that the subset $\alpha(r, t)$ consists of all internal lines of $D_{2}$ that run between vertices $r$ and $t$ of $D_{2}$. The subset $s$ of the internal lines of $D_{1}$ separates, accordingly, into a set of disjoint subsets $s_{\alpha}$. Two such subsets $s_{\alpha}{ }^{\prime}$ and $s_{\alpha}{ }^{\prime \prime}$ are said to be strongly equivalent if $s_{\alpha}{ }^{\prime}$ and $s_{\alpha}{ }^{\prime \prime}$ define the same set of flow lines and the sum of the (rest) masses associated with the lines in $s_{\alpha}{ }^{\prime}$ is the same as that in $s_{\alpha}{ }^{\prime \prime}$.

\section{Definition 1. 2.}

$D_{1}$ is said to contract to $D_{2}$ in an essentially unique way if the following conditions are satisfied: 
There is one subset $s^{\prime}$ of the internal lines of $D_{1}$ that map one-toone and onto the internal lines of $D_{2}$ in the manner described in Definition 1.1 and for any other such subset $s^{\prime \prime}$ the subsets $s_{\alpha}{ }^{\prime}$ and $s_{\alpha}{ }^{\prime \prime}$ are strongly equivalents for all $\alpha$.

\section{Definition 1.3 .}

A signed Landau diagram $D_{1}$ is said to contain a signed Landau diagram $D_{2}$ if and only if there is a correspondence of the internal lines of $D_{1}$ and $D_{2}$ that satisfies the conditions required in Definition 1.1 except for the connectedness requirement on the contracted part of $D_{1}$.

The notion that $D_{1}$ contains $D_{2}$ in an essentially unique way is defined in complete analogy to Definition 1.2.

\section{Example.}
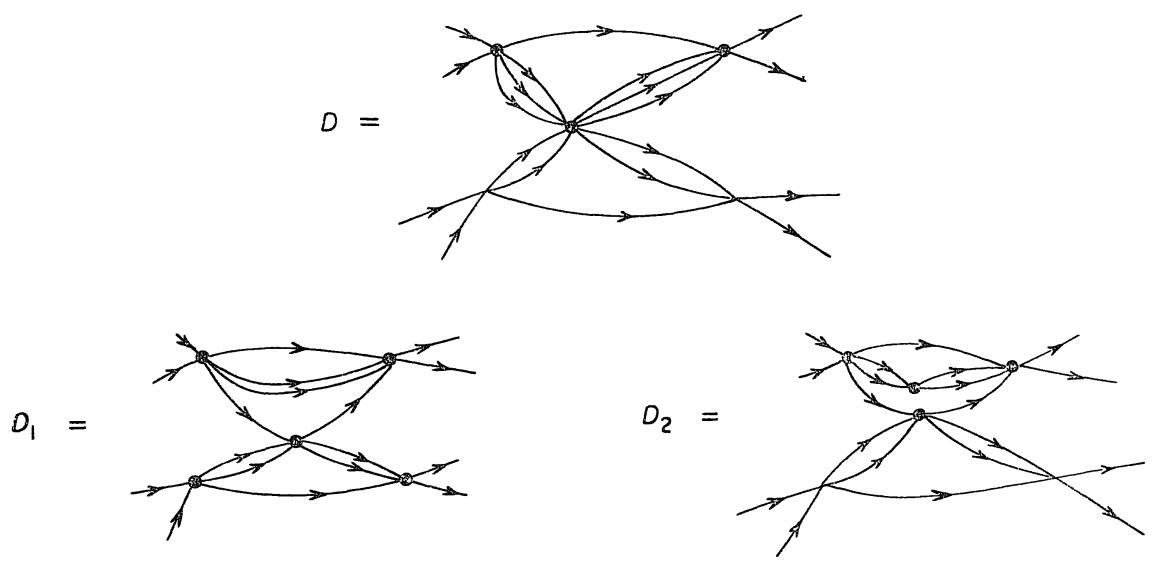

$D_{2}$ contains $D$, but $D_{1}$ does not. Neither $D_{1}$ nor $D_{2}$ contracts to $D$.

Using these definitions we can state the definition of the set $\mathcal{L}_{2}\left(D^{+}\right)$.

\section{Definition 1.4.}

$\mathcal{L}_{2}\left(D^{+}\right) \equiv\left\{(p ; u) \in \mathcal{L}_{0}\left(D^{+}\right) ;(p ; u)\right.$ lies in $\mathcal{L}\left(D_{1}^{+}\right)$only if $D_{1}^{+}$ contains $D^{+}$in an essentially unique way.\}

The central part of the derivation of Coster-Stapp [19] of general 
$S$-matrix discontinuity formula constitutes a proof that for any $D^{+}$and any point $\left(p_{0} ; \sqrt{-1} u_{0}\right)$ in $\mathcal{L}_{2}\left(D^{+}\right)$there is a neighborhood of $\left(p_{0} ; \sqrt{-1} u_{0}\right)$ in which one can derive solely from the unitarity and cluster decomposition properties of the $S$-matrix, an equation

$$
S=T\left(D^{+}\right)+R\left(D^{+}\right) \text {microlocally near } \mathcal{L}_{2}\left(D^{+}\right),
$$

where $T\left(D^{+}\right)$and $R\left(D^{+}\right)$have the properties described below.

The function $T\left(D^{+}\right)$has the form

$$
T\left(D^{+}\right) \equiv \int \prod_{b \in D^{+}} S^{b} \prod_{\substack{\alpha(r, t) \in D^{+} \\ \alpha(r, t) \neq \phi}} S_{\alpha(r, t)}^{-1} \prod_{l}\left(\frac{d^{4} k_{l}}{(2 \pi)^{4}}\right)
$$

For each vertex $b$ of $D^{+}$the quantity $S^{b}$ is the $S$-matrix corresponding to the process indicated by $b$. The incoming and outgoing particles for this process correspond to the incoming and outgoing lines of $b$, respectively. For each pair of vertices $\left(b_{r}, b_{t}\right)$ of $D^{+}$the set $\alpha(r, t)$ is the set of lines of $D^{+}$that join $b_{r}$ to $b_{t}$, and $\mathcal{H}_{\alpha(r, t)}$ is the Hilbert space corresponding to the associated set of particles. The quantity $S_{\alpha}$ is the restriction of $S$ to $\mathscr{H}_{\alpha}$ and $S_{\alpha}{ }^{-1}$ is the inverse in $\mathcal{H}_{\alpha}$ of $S_{\alpha}$. The suppressed arguments of the factors $S^{b}$ and $S_{\alpha}^{-1}$ are the variables $p_{r}$ and $k_{l}$ associated with the appropriate lines of the diagram exemplified below, and there is a mass-shell integration over all internal variables $k_{l}$. The phase space factors $(2 \pi)^{-3} \delta^{+}\left(k_{l}{ }^{2}-m_{l}{ }^{2}\right) d^{4} k_{l}$ are the same as for the bubble diagram functions. These integrations bring the product (1.2) to the form of a product of partially ordered operators.

\section{Example.}

Let $D$ be the diagram

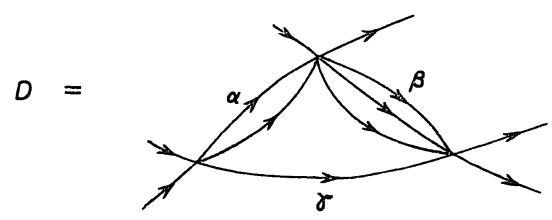

where each line is associated with some particular particle, and the letters $\alpha, \beta$ and $\gamma$ label the sets of lines joining pairs of vertices. Then the 
function $T\left(D^{+}\right)$is represented by the diagram

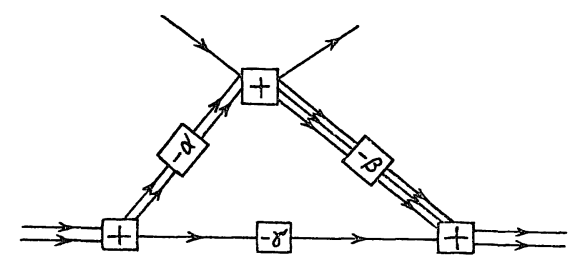

Note that if the set $\gamma$ consists of only one line, as in this example, then $S_{\gamma}^{-1}$ is nothing but the operator defined by the kernel function $2 \pi \delta^{+}\left(k_{l}{ }^{2}-m_{l}{ }^{2}\right)$, where $m_{l}$ is the mass of the particle associated with line $l$ of $D$.

\section{Definition 1.5 .}

A Landau diagram $D_{1}$ is said to fit into $T\left(D^{+}\right)$if and only if $D_{1}$ fits into some bubble diagram that occurs (with nonzero coefficient) in the sum obtained by applying the cluster decomposition: (i) to each function $S^{b}$ corresponding to a vertex $b$ of $D^{+}$(or to a plus box $b$ of the corresponding diagram, as in the example) and (ii) to each function $R_{\alpha}=S_{\alpha}-1$ in (1.2), where each $S_{\alpha}^{-1}$ is represented by the expansion $S_{\alpha}{ }^{-1}=\sum_{n=0}^{\infty}(-R)^{n} \quad$ (See Coster-Stapp [18] $\S 4$ for a discussion of certain cancellations that occur in this latter sum).

The function $R\left(D^{+}\right)$has the form

$$
R\left(D^{+}\right)=\sum_{B \in \mathscr{B}\left(R, D^{+}\right)} F^{B}
$$

where $\mathscr{B}\left(R, D^{+}\right)$is a set of bubble diagrams with the following property:

(1.4) No $D_{1}$ that contains $D^{+}$fits into any $B \in \mathscr{B}\left(R, D^{+}\right)$.

This property (1.4) of $\mathscr{B}\left(R, D^{+}\right)$, in conjunction with the Structure Theorem described in $\S 0$, shows that if only positive- $\alpha$ diagrams need be considered in calculating the singularity spectrum of $R\left(D^{+}\right)$near $\mathcal{L}_{2}\left(D^{+}\right)$then the following formula holds:

\section{The Microlocal Discontinuity Formula}

$$
S=T\left(D^{+}\right) \text {microlocally near } \mathcal{L}_{2}\left(D^{+}\right) .
$$


Several methods have been used to justify the assumption that only positive- $\alpha$ diagrams need be considered in calculating the singularity spectrum of $R\left(D^{+}\right)$near $\mathcal{L}_{2}\left(D^{+}\right)$. One is to take this assumption as an ansatz, and then show, using (1.5), that all singularities of $R\left(D^{+}\right)$near $\mathcal{L}_{2}\left(D^{+}\right)$not associated with positive- $\alpha$ diagrams cancel among themselves. This cancellation has been confirmed in many special cases, but no general proof has been given.

This assumption that the singularities associated with nonpositive- $\alpha$ diagrams give no net contribution to the singularity spectrum of $R\left(D^{+}\right)$ near $\mathcal{L}_{2}\left(D^{+}\right)$is the strong version of the mixed- $\alpha$ cancellation assumption.

A weaker version of the mixed- $\alpha$ cancellation assumption has been introduced by Coster-Stapp [19]. This assumption is that in equation (1.1) [or more generally in any equation $\sum F^{B}=0$ derived solely from the unitarity and cluster decomposition properties of the $S$-matrix] the singularities associated with all positive- $\alpha$ diagrams cancel among themselves, as do the singularities associated with all nonpositive- $\alpha$ diagrams. This assumption permits one to prove the desired result (i.e., that the singularities of $R\left(D^{+}\right)$associated with nonpositive- $\alpha$ singularities cancel among themselves near $\mathcal{L}_{2}\left(D^{+}\right)$) by proving this result rather for $T\left(D^{+}\right)$, since $S$ has only positive- $\alpha$ singularities. This latter task is generally simpler than the former one, and is more amenable to general arguments since $T\left(D^{+}\right)$has a simple standard form.

An argument suggesting that $T\left(D^{+}\right)$has no net singularities associated with nonpositive- $\alpha$ diagrams has been given by Coster-Stapp [19]. However, a complete proof would require some additional assumptions.

The weak mixed- $\alpha$ cancellation assumption described above is a particular case of the general idea that in equations of the form $\sum F^{B}=0$ derived solely from unitarity and cluster decomposition there is never any cancellation between singularities associated with different diagrams: i.e., each singularity of bubble diagram function $F^{B}$ can be associated with some particular signed Landau $D$ that fits into $B$, and the singularities associated with each $D$ cancel out among themselves in the sum $\sum F^{B}$. In other words the association between singularities and digarams is invariant under manipulations that depend only on unitarity and cluster 
decomposition.

It is not obvious that the singularities associated with each diagram must cancel among themselves in such equations. On the other hand, it is probably impossible to satisfy all the interrelated conditions entailed by unitarity and microanalyticity if singularities associated with different diagrams must cancel against each other. For singularities associated with different diagrams generally have different characteristics, and these different characteristics make systematic cancellations among them difficult to achieve.

This idea that the singularities of each bubble diagram function $F^{B}$ can be apportioned among the diagrams $D$ that fit into $B$, and that the association between singularities and diagrams is not altered by manipulations involving only the unitarity and cluster decomposition properties of the $S$-matrix can be formulated as follows:

\section{The Principle of Separation of Singularities}

Near any point $\left(p_{0} ; \sqrt{-1} u_{0}\right)$ of $\sqrt{-1} S^{*} \mathcal{M}_{r}$ any bubble diagram function $F^{B}$ can be locally decomposed into a sum of microfunctions $F^{B}\left(D ; p_{0}, u_{0}\right)$, each one defined for all $(p ; \sqrt{-1} u)$ in some neighborhood of $\left(p_{0} ; \sqrt{-1} u_{0}\right)$, such that

(1.6a) $F^{B}=\sum_{D} F^{B}\left(D ; p_{0}, u_{0}\right)$ microlocally near $\left(p_{0} ; \sqrt{-1} u_{0}\right)$

(1.6b) $F^{B}\left(D ; p_{0}, u_{0}\right)=0$ unless $\left(p_{0} ; \sqrt{-1} u_{0}\right) \in \widetilde{L}(D)$ and $D \in \bar{B}$, where $\widetilde{\mathcal{L}}(D)$ is defined below.

Furthermore, if for some sets $I$ and $J$ of bubble diagrams one can derive the equations

(1.6 c) $\sum_{i \in I} F^{B_{i}}=\sum_{j \in J} F^{B_{j}}$ microlocally near $\left(p_{0} ; \sqrt{-1} u_{0}\right)$ solely from unitarity and cluster decomposition, then for every $D$

(1. 6d) $\sum_{i \in I} F^{B_{i}}\left(D ; p_{0}, u_{0}\right)=\sum_{j \in J} F^{B_{j}}\left(D ; p_{0}, u_{0}\right)$ microlocally near $\left(p_{0} ; \sqrt{-1} u_{0}\right)$.

That is, in equations of the form $\sum F^{B}=0$ derived solely from unitarity and cluster decomposition the singularities associated with each diagram $D$ cancel among themselves, not against singularities associated with other diagrams. 
The set $\widetilde{\mathcal{L}}(D)$ occurring in $(1.6 \mathrm{~b})$ is the set defined by KashiwaraKawai-Stapp [6]. However, for present purposes it can be identified with $\mathcal{L}(D)$.

This principle of separation of singularities can be used in the following way. Suppose one can derive solely from unitarity and cluster decomposition a set of equations

(1.7 a) $\quad \sum_{i \in I_{1}} F^{B_{i}}=\sum_{i \in I_{2}} F^{B_{i}}=\cdots \quad$ microlocally near $\left(p_{0} ; \sqrt{-1} u_{0}\right)$.

And suppose for every signed $D$ satisfying $\left(p_{0} ; \sqrt{-1} u_{0}\right) \in \widetilde{L}(D)$ there is some index $n(D)$ such that for every $i$ in $I_{n}(D)$

$$
D \notin \bar{B}_{i} .
$$

Then separation of singularities implies, for each $n$, that

(1.7 c) $\quad \sum_{i \in I_{n}} F^{B_{i}}=0$ microlocally near $\left(p_{0} ; \sqrt{-1} u_{0}\right)$

The points $(p ; \sqrt{-1} u)$ studied in the present article are limited to cases such that the sets of lines $\alpha(r, t)$ of the relevant diagrams contain at most two lines, and such that the sum of the masses of the corresponding pairs of particles lies below the lowest threshold for the production of three or more communicating particles. The remainder of this section is devoted to showing how the microlocal discontinuity formula can be derived from the principle of separation of singularities for the cases needed in the present work.

From unitarity and cluster decomposition one can derive (Coster-Stapp [18]) the following four equations:

$$
\begin{aligned}
S-T\left(D^{+}\right) & =S-T^{R}\left(D^{+}\right) \\
& =S-T^{L}\left(D^{+}\right) \\
& =R\left(D^{+}\right) \quad \text { microlocally near } \mathcal{L}_{2}\left(D^{+}\right) .
\end{aligned}
$$

Here $T^{R}\left(D^{+}\right)$is the same as $T\left(D^{+}\right)$except that all the factors $S_{\alpha}{ }^{-1}$ are eliminated by multiplying them into the factors $S^{b}$ that stands on their right. These $S^{b}$ then become the left-truncated functions (Stapp [5]). The $T^{L}\left(D^{+}\right)$is similarly defined, with left and right interchanged.

We wish to show, for any $\left(p_{0} ; \sqrt{-1} u_{0}\right) \in \mathcal{L}_{2}\left(D^{+}\right)$, and any signed diagram $D_{1}$ satisfying $\left(p_{0} ; \sqrt{-1} u_{0}\right) \in \mathcal{L}\left(D_{1}\right)$ that there is one of the 
four members of (1.8) such that $D_{1}$ fits into no $B_{i}$ that corresponds to a term $F^{B_{i}}$ that occurs in that member. That is, no signed diagram $D_{1}$ fits into all four members of (1.8). Once this is shown then (1.7) implies the microlocal discontinuity formula

$$
\begin{aligned}
S-T\left(D^{+}\right) & =S-T^{R}\left(D^{+}\right) \\
& =S-T^{L}\left(D^{+}\right) \\
& =R\left(D^{+}\right)=0 \text { microlocally on } \mathcal{L}_{2}\left(D^{+}\right) .
\end{aligned}
$$

All positive- $\alpha$ diagrams $D_{1}{ }^{+}$are immediately eliminated by the fact that the only positive $-\alpha$ diagrams $D_{1}^{+}$such that $\mathcal{L}\left(D_{1}^{+}\right) \cap \mathcal{L}_{2}\left(D^{+}\right) \neq \phi$ are $D_{1}^{+}$that contain $D^{+}$, by the definition of $\mathcal{L}_{2}\left(D^{+}\right)$, and no such $D_{1}^{+}$ fits into $\mathscr{B}\left(R, D^{+}\right)$, by construction.

No nonpositive- $\alpha$ diagram fits into the diagrams that represent $S$. Thus it is sufficient to show that no signed $D_{1}$ with a negative sign $\sigma_{l}$ fits into $T\left(D^{+}\right), T^{R}\left(D^{+}\right)$and $T^{L}\left(D^{+}\right)$.

The condition that $D_{1}$ fit into $T\left(D^{+}\right)$entails that $D_{1}$ contain a signed diagram $D$ that is equal to $D^{+}$except for signs. Thus $D_{1}$ must have at least one cut $C_{\alpha}$. A cut $C_{\alpha}$ is a set of lines of $D_{1}$ that corresponds to the set $\alpha=\alpha(s, t)$ of $D$ in a correspondence that shows that $D_{1}$ contains $D$. The diagram $D_{1}$ may have several cuts strongly equivalent to $C_{\alpha}$.

The requirement that $D_{1}$ fit into $T\left(D^{+}\right)$entails (below the threshold for the production for three or more particles in the channel defined by $C_{\alpha}$ ) that every minus line of $D_{1}$ lie on a cut strongly equivalent to a cut $C_{\alpha}$. The requirement that $D_{1}$ lies in $T^{R}\left(D^{+}\right)$implies that no cut strongly equivalent to $C_{\alpha}$ can have all positive lines and stand to the right of a strongly equivalent cut that contains a minus line. This follows from the defining properties of the left-truncated functions. Similarly the requirement that $D_{1}$ fit into $T^{L}\left(D^{+}\right)$entails that no cut strongly equivalent to $C_{\alpha}$ can have all positive lines and stand to the left of a strongly equivalent cut that contains a minus line. Thus no cut strongly equivalent to $C_{\alpha}$ can have all positive lines unless every cut strongly equivalent to $C_{\alpha}$ has all positive lines. Since at least one cut strongly equivalent to some $C_{\alpha}$ must contain a minus line there must be some cut $C_{\alpha}$ such that every cut equivalent to it contains a minus 
line. In our case, where each cut equivalent to any cut $C_{\alpha}$ contains at most two lines, this means that the removal of the trivial vertices that separate the various cuts equivalent to each $C_{\alpha}$ will reduce $D_{1}$ to a diagram $D_{2}$ that has precisely one cut $C_{\alpha}$ equivalent to each set $\alpha$ of $D^{+}$, and one of these cuts $C_{\alpha}$ must contain a minus line. Moreover, every minus line in $D_{2}$ must lie on one of these cuts $C_{\alpha}$. But then the argument used to prove Theorem 0.0 shows that $D_{2}$ can be changed into a positive- $\alpha$ diagram $D_{3}{ }^{+}$such that $\left(p_{0} ; \sqrt{-1} u_{0}\right)$ lies on $\mathcal{L}\left(D_{3}{ }^{+}\right)$, but such that $D_{3}{ }^{+}$does contain $D^{+}$. This conclusion contradicts the requirement that $\left(p_{0} ; \sqrt{-1} u_{0}\right)$ lies on $\mathcal{L}_{2}\left(D^{+}\right)$. Thus there can be no signed diagram $D_{1}$ that fits into all four members of (1.8). Hence the microlocal discontinuity formula (1.9) used in the present work follows from the unitarity of $S$, the microanalyticity postulate, and the principle of separation of singularities. This proof circumvents both the strong version of the mixed- $\alpha$ cancellation assumption, which has no a priori justification, and the failure of the structure theorem at $u=0$ points.

This use of the principle of separation of singularities to prove the $S$-matrix discontinuity formula provides an alternative to the method based on a stronger analyticity assumption (see §5) and improved Structure Theorem (Kashiwara-Kawai-Stapp [6]).

\section{$\S 2$. Verification of Sato's conjecture for simple diagrams}

In this section we prove the holonomicity of the $S$-matrix near points where only simple diagrams are relevant.

\section{$\S 2.1$. Study of the holonomic structure near a point where only a single simple Landau diagram $D^{+}$is relevant}

We begin our investigation by proving the following Theorem 2.1.1. The case covered by this theorem is rather simple, but the results obtained here will be used later.

\section{Definition 2.1. 1 .}

Let $D$ be a simple diagram. Then a point $p$ in $L\left(D^{+}\right)$is said to be elementary with respect to $D^{+}$if and only if the following condition 
is satisfied:

Let $k_{l}(p)$ be the unique*) value of $k_{l}$ that permits a solution at $p$ of the Landau equations associated with $D^{+}$. And for each vertex $b$ of $D$ define the corresponding set of vectors

$$
p^{b} \equiv\left\{\left(p_{r}, k_{l}(p)\right) ;[b: r] \neq 0,[b: l] \neq 0\right\} .
$$

Then for every $b$ the following condition holds:

$$
p^{b} \notin L^{+}
$$

That is, each $b$ the point $p^{b}$ is a regular point of the scattering function $s^{b}$ occurring in $T\left(D^{+}\right)$.

The set of all $p \in L\left(D^{+}\right)$that are elementary with respect to $D^{+}$ is denoted by $L_{e}\left(D^{+}\right)$.

\section{Theorem 2.1. 1 .}

Let $p_{0}$ be a point in $\mathscr{M}-\mathcal{M}_{0}$. Assume that

(i) there is a unique unsigned $D$ such that $p_{0}$ lies on $L_{0}\left(D^{+}\right)-M_{0}$.

(ii) This diagram $D$ is simple and connected.

(iii) $p_{0}$ lies on $L_{e}\left(D^{+}\right)$.

Then the $S$-matrix $S(p)$ satisfies a simple holonomic system $\mathfrak{M}$ of microdifferential equations on $\sqrt{-1} S^{*} W$ in a neighborhood $W$ of $p_{0}$ in $M$. The characteristic variety of $\mathfrak{M}$ is the set $\left\{(p ; u) ; p \in W \cap L_{0}{ }^{C}(D)\right.$, $u=\operatorname{grad}_{p} \phi(p)$, where $\phi(p)=0$ defines $L_{0}(D)$ near $\left.p_{0}\right\}$, and the order of $\mathfrak{M}$ is $\alpha=\alpha(D)=-\frac{3}{2} N+2 n^{\prime}$, where $N$ denotes the number of internal lines of $D$ and $n^{\prime}$ denotes the number of vertices of $D$.

Proof.

Since $p_{0}$ belongs to $L_{0}\left(D^{+}\right)$for a unique Landau diagram $D^{+}$, which is connected, the $S$-matrix at $p_{0}$ is equal to its connected part. For if $S_{c}$ were different from $S$ at $p_{0}$, then this point would have to lie on $L\left(D_{1}^{+}\right)$in a disconneted diagram $D_{1}^{+}$, contrary to assumption.

Since $p_{0}$ lies on $L_{0}\left(D^{+}\right)-\mathscr{M}_{0}$, we can find a real-valued real analytic

* Note that $k_{2}$ is uniquely determined by $p$ at any positive- $\alpha$ Landau point. See the proof of Theorem 0.0. See also the proof of Theorem 2.1.4. below. 
function $\phi(p)$ so that $L_{0}\left(D^{+}\right)=\{p \in \mathscr{M} ; \phi(p)=0\}$ and $u=\operatorname{grad}_{p} \phi(p)$ in a neighborhood of $p_{0}$. Note that the micro-local result automatically proves the local result in this case, because a point $p$ in $L_{0}\left(D^{+}\right)$near $p_{0}$ determines a unique point in $\mathcal{L}\left(D^{+}\right)$.

Condition (i) of the theorem insures that $\left(p_{0} ; \operatorname{grad}_{p} \phi\left(p_{0}\right)\right)$ lies on $\mathcal{L}_{2}\left(D^{+}\right)$, since if $p_{0}$ lies on $L\left(D^{+}\right)$for some diagram that does not contain $D^{+}$it must lie on $L_{0}\left(D_{3}^{+}\right)$for some $D_{3}{ }^{+} \neq D^{+}$. (See Chandler-Stapp [7] Theorems 5 and 6).

Now the microlocal discontinuity formula tells us that the $S$-matrix (regarded as a microfunction) is equal to the threshold function $T\left(D^{+}\right)$ near $(p ; \sqrt{-1} u) \in \mathcal{L}_{2}\left(D^{+}\right)$. Since the diagram $D$ is simple, $T\left(D^{+}\right)$has the form

$$
\int \prod_{j=1}^{n^{\prime}} S_{j}(p, k) \prod_{l=1}^{N} \delta^{+}\left(k_{l}{ }^{2}-m_{l}{ }^{2}\right) \prod_{l=1}^{N} d^{4} k_{l}
$$

Here $S_{j}(p, k)$ is the $S$-matrix inserted at vertex $j$ of $D$. Since $p_{0}$ lies on $L_{e}\left(D^{+}\right)$this integral (2.1.1) is evaluated at points where $S_{j}(p, k)$ has the form $(2 \pi)^{4} \delta^{4}\left(\sum_{r}[j: r] p_{r}+\sum_{l}[j: l] k_{l}\right) s_{j}(p, k)$ with a real analytic function $s_{j}(p, k) .^{*}$ Therefore it suffices to show that

$$
\int \prod_{j=1}^{n^{\prime}} s_{j}(p, k) \prod_{j=1}^{n^{\prime}} \delta^{4}\left(\sum_{r}[j: r] p_{r}+\sum_{l}[j: l] k_{l}\right) \prod_{l=1}^{N} \delta^{+}\left(k_{l}{ }^{2}-m_{l}{ }^{2}\right) \prod_{l=1}^{N} d^{4} k_{l}
$$

satisfies a holonomic system of micro-differential equations in a neighborhood of $p_{0}$. In order to see this, we first show that the integrand satisfies a holonomic system. Once this is shown, then Theorem 3.5.5 of S-K-K [2] Chapter II can be applied to prove the existence of a holonomic system that the integral must satisfy. Since $\prod_{j=1}^{n^{\prime}} s_{j}(p, k)$ is an analytic function, it suffices to show that

$$
\Delta=\prod_{j=1}^{n^{\prime}} \delta^{4}\left(\sum_{r}[j: r] p_{r}+\sum_{l}[j: l] k_{l}\right) \prod_{l=1}^{N} \delta^{+}\left(k_{l}{ }^{2}-m_{l}{ }^{2}\right)
$$

is holonomic. An important step in showing this property is the following lemma:

* Strictly speaking $s_{j}(p, k)$ is defined only on the mass-shell manifold within the framework of the on-shell $S$-matrix theory. However, any analytic extension of $s_{j}(p, k)$ off mass-shell gives the same result, because the mass-shell delta functions $\delta^{+}\left(k_{l}{ }^{2}-m_{l}{ }^{2}\right)$ eliminate all off-shell contributions. 


\section{Lemma 2.1.2.}

Let $\phi(x)$ be a real-valued real analytic function defined on a real analytic manifold $M$. Denote by $X$ the complexification of $M$ upon which $\phi(x)$ is defined. Assume that $\operatorname{grad}_{x} \phi(x)$ never vanishes on $H=\{x \in X ; \phi(x)=0\}$. Then $f(x)=\delta(\phi(x))$ is a solution of simple holonomic system $\mathfrak{M}_{\phi}$ of (micro-)differential equations whose characteristic variety $V_{\phi}$ is given by the conormal bundle $P_{H}{ }^{*} X$ supported by $H$, i.e., $\left\{(x ; \eta) \in P^{*} X ; \phi(x)=0, \eta=c \operatorname{grad}_{x} \phi(x), c \neq 0\right\}$. Its order*) is $1 / 2$.

\section{Proof of Lemma 2.1.2.}

Since the problem is of local character, one may assume $\phi(x)=x_{1}$ for a local coordinate system $\left(x_{1}, \cdots, x_{n}\right)$. Then it is clear that $f=\delta\left(x_{1}\right)$ satisfies the following holonomic system

$$
\mathfrak{W}:\left\{\begin{array}{l}
x_{1} f=0 \\
\frac{\partial}{\partial x_{j}} f=0 \quad j=2, \cdots, n .
\end{array}\right.
$$

Then all the assertions of the lemma are obvious.

Q.E.D.

\section{Proof of Theorem 2.1.1. continued.}

We now apply Proposition 4.2.4 (4) and (2) of S-K-K [2] Chapter II (or Theorem 3.5.9 and Theorem 3.5.3 of S-K-K [2] Chapter II) to conclude that $\Delta$ satisfies locally a simple holonomic system of (micro-) differential equations. The conditions of the above quoted propositions are clearly satisfied under the following condition:

(2.1.3) For all $p$ in some complex neighborhood of $p_{0}$ the real surfaces

$$
\begin{aligned}
& p_{r}{ }^{2}=\mu_{r}{ }^{2}, p_{r, 0}>0(r=1, \cdots, n), k_{l}{ }^{2}=m_{l}{ }^{2}, k_{l, 0}>0(l=1, \cdots, N) \text { and } \\
& \sum_{r}[j: r] p_{r}+\sum_{l}[j: l] k_{l}=0 \quad(j=1, \cdots, n) \text { cross transversally. }
\end{aligned}
$$

This transversality property follows from Theorems 5 and 6 of Chandler-Stapp [7]. If $p_{0} \in L\left(D^{+}\right)-\mathscr{M}_{0}$ for a unique diagram $D^{+}$then (2.1.3) is true, unless there is at $p=p_{0}$ a $u \equiv\left(u_{1}, \cdots, u_{n}\right)=0$ solution,

* See S-K-K [2] Chapter II $\S 4.2$ for the definition of the order of (simple) holonomic (=maximally overdetermined) system. 
with some $\alpha_{l} \neq 0$ or some $\beta_{r} \neq 0$, of the following equations:

$$
\begin{cases}\sum_{r=1}^{n}[j: r] p_{r}+\sum_{l=1}^{N}[j: l] k_{l}=0 & j=1, \cdots, n^{\prime} \\ p_{r}{ }^{2}=\mu_{r}{ }^{2}, p_{r, 0}>0 & r=1, \cdots, n \\ k_{l}{ }^{2}=m_{l}{ }^{2}, k_{l, 0}>0 & l=1, \cdots, N \\ \sum_{j=1}^{n^{\prime}}[j: l] v_{j}=\alpha_{l} k_{l} & l=1, \cdots, N \\ u_{r}=-[j(r): r]\left(v_{j(r)}+\beta_{r} p_{r}\right) & r=1, \cdots, n\end{cases}
$$

These equations are just the Landau equations given in $\S 0$. According to Theorems 5 and 6 of Chandler-Stapp [7] these equations admit no strictly positive- $\alpha$ or strictly negative- $\alpha u=0$ solution for any $p \in \mathscr{M}-\mathscr{M}_{0}$. Thus they admit no such solution at $p_{0}$. Hence it suffices to show that they admit no mixed- $\alpha$ or zero- $\alpha$ solution $(p ; u)$ $=\left(p_{0} ; 0\right)$.

Actually, (the proof of) Theorem 6 of Chandler-Stapp [7] shows that the $\alpha$ 's in (2.1.4) are uniquely determined up to a single overall factor at any point of $L_{0}\left(D^{+}\right)-\mathcal{M}_{0}$. Thus no mixed- $\alpha$ solutions are allowed at these points, since a positive- $\alpha$ solution (with $u \neq 0$ ) is present. On the other hand, if all $\alpha_{l}=0$, then all the $v_{j}$ are equal, and, the condition $u=0$ implies, for $p \notin \mathcal{M}_{0}$, that all $\beta_{r}=0$. Thus the transversality condition is satisfied, and the above-quoted proposition of S-K-K [2] combined with Lemma 2.1.2 shows that $\Delta$ satisfies, locally, for all $p$ sufficiently close to $p_{0}$ and all $k$ with $\operatorname{Re} k_{l, 0}>0$, a simple holonomic system of order $\alpha(\Delta)=\frac{1}{2} \times 4 n^{\prime}+\frac{1}{2} N$.

The characteristic variety of the holonomic system $\mathfrak{M}$ that $\Delta(p, k)$ satisfies, and hence that of the holonomic system $\widetilde{\mathfrak{M}}$ that $\prod_{j=1}^{n^{\prime}} s_{j}(p, k) \Delta(p, k)$ satisfies near $p_{0}$, is given by the following equations considered in $P^{*} Y$, where $Y$ denotes the complex manifold over which $(p, k)$ runs. Here $\left.u_{r}^{*}\right)$ ( $w_{l}$, resp.) stands for the cotangent vector corresponding to $p_{r}\left(k_{l}\right.$, resp.).

* Precisely speaking, $u_{r}$ is a representative of a cotangent vector corresponding to $p_{r}$, since $p_{r}$ is confined to the mass-shell manifold $\mathcal{M}=\left\{p_{r}{ }^{2}=\mu_{r}{ }^{2}, r=1, \cdots, n\right\}$. See $\S 0$. 
$(2.1 .5)$

$$
\begin{cases}\sum_{r=1}^{n}[j: r] p_{r}+\sum_{l=1}^{N}[j: l] k_{l}=0 & j=1, \cdots, n^{\prime} \\ p_{r}{ }^{2}=\mu_{r}{ }^{2}, \quad \operatorname{Re} p_{r, 0}>0 & r=1, \cdots, n \\ k_{l}{ }^{2}=m_{l}{ }^{2}, \quad \operatorname{Re} k_{l, 0}>0 & l=1, \cdots, N \\ v_{l}-\sum_{j=1}^{n}[j: l] v_{j}+\alpha_{l} k_{l}=0 & l=1, \cdots, N \\ u_{r}=-[j(r): r]\left(v_{j(r)}-\beta_{r} p_{r}\right) & r=1, \cdots, n\end{cases}
$$

Next we use Proposition 4.2.4 (3) (or Theorem 3.5.5) of S-K-K [2] Chapter II to show that the integral $T\left(D^{+}\right)$satisfies near $p_{0}$ a simple holonomic system of micro-differential equations. In order to see this, it suffices to show that the $\alpha_{l}$ and $k_{l}$ are uniquely determined by $(p ; u)$ on the intersection of the variety defined by (2.1.5) with $w_{l}=0(l=1$, $\cdots, N)$ in the sense that $\alpha_{l}$ and $k_{l}$ are determined by the implicit function theorem. This is exactly what Chandler-Stapp [7] have shown (p. 852) under the conditions of Theorem 2.1.1, since the intersection of the variety given by $(2.1 .5)$ and $w_{l}=0(l=1, \cdots, N)$ is equivalent to the complexification of the Landau equations (2.1.4). Note that the proof of Chandler-Stapp [7] holds without any change in a sufficiently small complex neighborhood of the real point $p_{0}$ in question, because the rank condition used there is an open condition. This proves that $T\left(D^{+}\right)$ satisfies near $p_{0}$ a simple holonomic system of micro-differential equations whose characteristic variety is given by (2.1.5). Furthermore, Proposition 4.2.4 (3) of S-K-K [2] Chapter II tells us its order $\alpha \equiv \alpha(D)$ is given by $\alpha(\Delta)-\frac{1}{2} \times 4 N=2 n^{\prime}-\frac{3}{2} N$. This completes the proof of Theorem 2.2.1.

Remark.

In Theorem 2.1.1 we discussed the $S$-matrix itself. However, the scattering amplitude $s(p)$ can be discussed without any essential changes of the argument. The differences needed are the following:

Since $s(p)$ is considered on the reduced mass-shell manifold $\mathcal{M}_{r}$, equation (2.1.5e), hence (2.1.4 e), should be replaced by (2.1. $\left.4 \mathrm{e}^{\prime}\right) \quad u_{r}=-[j(r): r]\left(v_{j(r)}+\beta_{r} p_{r}+a\right)$ with some four-vector $a$. 
(Cf. $\left.\left(0.2^{\prime}\right).\right)$

Also, as an effect of the absence of the over-all $\delta$-function, the order of the system involved must be reduced by 2 .

This remark should apply to all cases discussed below.

An important corollary of Theorem 2.1.1 is the following result which specifies the explicit form of singularities of the scattering matrix $S(p)$.

\section{Corollary 1 .}

Let $\phi(p)$ be a local defining function of $L_{0}\left(D^{+}\right)$such that at points $p$ in $W \cap\{p \in \mathscr{M} ; \phi(p)=0\}$ the pair $(p ; u)=\left(p ; \operatorname{grad}_{p} \phi(p)\right)$ satisfies the Landau equations. Then $S(p)$ has the following form in a real neighborhood of a point $p_{0}$ satisfying the conditions of the theorem:

$$
\begin{aligned}
& \delta^{4}\left(\sum_{j, r}[j: r] p_{r}\right)\left(h_{1}(p)(\phi(p)+i 0)^{-\alpha+\frac{3}{2}}+h_{2}(p)\right) \\
& \text { if }-\alpha+\frac{3}{2} \text { is neither a positive integer nor zero }
\end{aligned}
$$

or

$$
\begin{aligned}
& \delta^{4}\left(\sum_{j, r}[j ; r] p_{r}\right)\left(h_{1}(p) \phi(p)^{-\alpha+\frac{3}{2}} \log (\phi(p)+i 0)+h_{2}(p)\right) \\
& \text { if }-\alpha+\frac{3}{2} \text { is a positive integer or zero. }
\end{aligned}
$$

Here $h_{1}(p)$ and $h_{2}(p)$ are analytic functions.

Proof.

Since $L_{0}\left(D^{+}\right)$is of codimension 1 in $\mathscr{M}_{r}$, the scattering amplitude $s(p)$ must have the following form. (Theorem 4.2.5 of S-K-K [2] Chapter II.)

(2. 1.7)

$$
\left\{\begin{array}{l}
h_{1}(p)(\phi(p)+i 0)^{\lambda}+\tilde{h}_{1}(p)(\phi(p)-i 0)^{\lambda}+ \\
\quad+c_{\lambda}\left(k_{1}(p) \log (\varphi(p)+i 0)+\tilde{k}_{1}(p) \log (\varphi(p)-i 0)\right)+h_{2}(p) \\
\quad \text { if } \lambda \text { is neither a positive integer nor zero, where } c_{\lambda}=0 \\
\quad \text { unless } \lambda \text { is a negative integer } \\
\text { or } \\
h_{1}(p) \phi(p)^{\lambda} \log (\phi(p)+i 0)+\tilde{h}_{1}(p) \phi(p)^{\lambda} \log (\phi(p)-i 0)+h_{2}(p) \\
\quad \text { if } \lambda \text { is either a positive integer or zero. }
\end{array}\right.
$$


Here $\lambda=-(\alpha(D)-2)-\frac{1}{2}=-\alpha(D)+\frac{3}{2}$ and $h_{1}(p), \tilde{h}_{1}(p) k_{1}(p), \tilde{k}_{1}(p)$ and $h_{2}(p)$ are analytic. Furthermore, one can easily verify that $c_{\lambda}=0$ in view of the support property of $T\left(D^{+}\right)$.

On the other hand, S.S. $S(p)$ is contained in $\mathcal{L}\left(D^{+}\right)$if $p$ lies in a neighborhood of $p_{0}$. (Microanalyticity postulate on the $S$-matrix: See Chandler-Stapp [7], Iagolnitzer-Stapp [8] and Kashiwara-Kawai-Stapp [6].) Therefore, $\tilde{h}_{1}(p)$ must be identically zero. This proves Corollary 1.

Remark.

This result shows, a posteriori, that the holonomic system $\mathfrak{M}$ that the $S$-matrix satisfies near $p_{0}$ can be chosen to be a holonomic system of linear differential equations.

\section{Corollary 2.}

Under the conditions of Theorem 2.1.1 the singularity of the scattering amplitude $s(p)$ is at most a simple pole, i.e., it is a logarithmic singularity or a square root singularity (more precisely, at most a simple inverse power of a square root singularity) or a simple pole.

Proof.

Because of the transversality condition (2.1.3), the dimension of the following manifold $\mathrm{M}$ is $4 n+4 N-4 n^{\prime}-n-N=3 n+3 N-4 n^{\prime}$.

$$
\begin{aligned}
& \mathrm{M}=\left\{(p, k) \in \mathbb{R}^{4 n+4 N} ; \sum_{r}[j: r] p_{r}+\sum_{l}[j: l] k_{l}=0 \quad\left(j=1, \cdots, n^{\prime}\right),\right. \\
& \left.p_{r}{ }^{2}=\mu_{r}{ }^{2} \quad(r=1, \cdots, n), k_{l}{ }^{2}=m_{l}{ }^{2} \quad(l=1, \cdots, N)\right\} .
\end{aligned}
$$

On the other hand, denoting by $\pi_{p}(\mathrm{M})$ the projection of $\mathrm{M}$ to $\mathbb{R}_{p}{ }^{4 n}$ we have $\operatorname{dim} \pi_{p}(\mathrm{M}) \leqq \operatorname{dim} \mathrm{M}$. Since $L_{0}\left(D^{+}\right)$is of codimension 1 in the reduced mass-shell manifold (Chandler-Stapp [7]), $\operatorname{dim} \pi_{p}(\mathrm{M})=3 n^{\prime}-5$.

Therefore we have

$$
3 n-5 \leqq 3 n+3 N-4 n^{\prime}
$$

This implies

$$
\alpha(D) \equiv 2 n^{\prime}-\frac{3}{2} N \leqq \frac{5}{2}
$$


This is the required result in view of Corollary $1 . \quad$ Q.E.D.

The way in which Theorem 2.1.1 is proved suggests that its microlocal version should follow from the microlocal form of the discontinuity formula. To prepare for a theorem giving this result we introduce the following definition.

Definition 2.1.2. (Natural point of $\mathcal{L}_{0}\left(D^{+}\right)$).

Let $\left(p_{0} ; u_{0}\right)$ be a point of $\mathcal{L}_{0}\left(D^{+}\right)$. Let the set of diagrams $\left\{D_{j}\right.$; $\left.\left.j=1, \cdots, j_{0}\right)\right\}$ consist of all the contractions of $D$ that satisfy $p_{0} \in L_{0}\left(D_{j}^{+}\right)$. Suppose the following three conditions are satisfied:

(a) The local defining functions $\phi_{j}(p)$ of the surfaces $L_{0}\left(D_{j}{ }^{+}\right)$ satisfy the condition that the vectors $\operatorname{grad} \phi_{j}\left(p_{0}\right)$ are linearly independent.

(b) There is a neighborhood $\omega$ of $\left(p_{0} ; u_{0}\right)$ such that $\pi\left(\mathcal{L}^{C}(D) \cap \omega\right)$ $\subset \bigcap_{j=1}^{j_{0}} L_{0} \boldsymbol{c}\left(D_{j}\right)$. (Note that the corresponding real relation is true.)

(c) For each complex solution of the Landau equations associated with $D$ such that $(p ; u) \in \mathcal{L}(D) \cap \omega$ the corresponding vectors $k_{l}$ satisfy for each $j$, and for all $l$ such that $\alpha_{l} \neq 0$ in the solution corresponding to $D_{j}$, the relation

$$
k_{l}=k_{l}^{(j)}(p) \text {. }
$$

Here $k_{l}{ }^{(j)}(p)$ is the value of the energy-momentum vector of line $L_{l}$ in the solution at $p$ corresponding to $D_{j}$. (Note that the corresponding real condition is true.)

If these conditions are satisfied then $\left(p_{0} ; u_{0}\right)$ is called a natural point of $\mathcal{L}_{0}\left(D^{+}\right)$.

\section{Theorem 2.1.2.'}

If $\left(p_{0} ; u_{0}\right)$ is a natural point of $\mathcal{L}_{0}\left(D^{+}\right)$then for some neighborhood $\omega$ of $\left(p_{0} ; u_{0}\right)$ the set $\mathcal{L}_{0}{ }^{c}(D) \cap \omega^{\prime}$ is a nonsingular manifold and $k_{l}$ and $\alpha_{l}(l=1, \cdots, N)$ are analytic functions of the local coordinates on this manifold. 
Proof.

By Pham's result used in the proof of Theorem $0.0 k_{l}^{\left(j_{l}\right)}(p)$ $=k_{l}^{\left(j_{l}^{\prime}\right)}(p)$ if $p$ lies in $L_{0}\left(D_{j_{l}}\right) \cap L_{0}\left(D_{j_{l}^{\prime}}\right)$ and $\alpha_{l}$ is different from zero in the solutions corresponding to both $D_{j_{l}}$ and $D_{j_{l}^{\prime}}$. Since $k_{l}^{\left(j_{l}\right)}(p)$ $\left(k_{l}^{\left(j_{l}^{\prime}\right)}(p)\right.$, resp. $)$ is analytic in $p$ for $p$ in $L_{0}\left(D_{j_{l}}\right)\left(L_{0}\left(D_{j_{l}}\right)\right.$, resp. $)$ and since $L_{0}\left(D_{j_{l}}\right)$ and $L_{0}\left(D_{j_{l}^{\prime}}\right)$ intersect transversally by assumption (a), $k_{l}^{\left(j_{l}\right)}(p)=k_{l}^{\left(j_{l}^{\prime}\right)}(p)$ holds for $p \in L_{0}^{c}\left(D_{j_{l}}\right) \cap L_{0}{ }^{C}\left(D_{j_{l}}\right)$. Therefore, the set of conditions (c) of Definition 2.1.2 are compatible.

Now define $U_{j}(p)=\operatorname{grad}_{p} \phi_{j}(p)$. The result of Chandler-Stapp [7] entails that there exists a unique $A_{j}=\left(\alpha_{1}{ }^{(j)}, \cdots, \alpha_{N}{ }^{(j)}\right)$ corresponding to $\left(p ; \operatorname{grad}_{p} \phi_{j}\right)$. Note that $\alpha_{l}^{(j)}=0$ in $A_{j}$ if $L_{l}$ is contracted to obtain $D_{j}$ from $D$. Furthermore, $\left(p ; \sum_{j=1}^{j_{0}} e_{j} U_{j}(p)\right)$ satisfies the Landau equation associated with $D$ for any $\left(e_{j}\right) \in C^{j_{0}}$, since the $u$ vector allowed by the Landau equation is linear with respect to $\alpha$ 's once $p$ 's and $k$ 's are fixed. The corresponding $(k, \alpha)$ is, then, clearly $\left(k(p), \sum_{j=1}^{j_{0}} e_{j} A_{j}\right)$. Therefore, $\mathcal{L}_{0}(D){ }^{\boldsymbol{C}}$ is nonsingular and $(k, \alpha)$ is analytic in the local coordinates $\left(x_{1}, \cdots, x_{i_{0}} ; e_{1}, \cdots, e_{j_{0}}\right)$ of the manifold $\mathcal{L}_{0}{ }^{C}(D)$ near $\left(p_{0} ; u_{0}\right)$. Here $x_{1}, \cdots, x_{i_{0}}$ are the local coordinates of the manifold $\bigcap_{j=1}^{j_{0}} L_{0}^{c}\left(D_{j}\right)$ near $p_{0}$

Q.E.D.

\section{Theorem 2.1.3.}

Let $p_{0}$ be a point in $\mathscr{M}-\mathscr{M}_{0}$. Assume that $\left(p_{0} ; \sqrt{-1} u_{0}\right) \in \sqrt{-1} S^{*} \mathscr{M}$ satisfies, the following conditions:

(2.1.11) (i) there is a unique unsigned $D$ such that $\left(p_{0} ; \sqrt{-1} u_{0}\right)$ lies in $\mathcal{L}_{0}\left(D^{+}\right)$.

(ii) This $D$ is simple and connected.

(iii) $p_{0}$ lies in $L_{e}\left(D^{+}\right)$.

(2.1.12) $\quad\left(p_{0} ; \sqrt{-1} u_{0}\right)$ is a natural point of $\mathcal{L}_{0}\left(D^{+}\right)$.

Then the S-matrix $S(p)$ satisfies a simple holonomic system $\mathfrak{M}$ of micro-diffrential equations in a neighborhood of $\left(p_{0} ; \sqrt{-1} u_{0}\right) \in \sqrt{-1} S^{*} \mathcal{M}$, and its order is $\alpha(D)=2 n^{\prime}-\frac{3}{2} N$. The characteristic variety of $\mathfrak{M}$ coincides locally with $\mathcal{L}^{c}(D)$. 
Proof.

The microlocal discontinuity formula tells us that the connected part of the $S$-matrix is given by $T\left(D^{+}\right)$in a neighborhood of $\left(p_{0} ; \sqrt{-1} u_{0}\right)$. Furthermore, $T\left(D^{+}\right)$has the form (2.1.2), under the assumption of the theorem. Then the corollary of Theorem 0.0 shows that $p_{0}$ is not a $u=0$ point for the diagram $D$. This implies that the integrand of $T\left(D^{+}\right)$satisfies a simple holonomic system of micro-differential equations.

On the other hand, $\alpha_{l}$ and $k_{l}$ are determined uniquely in terms of $p$ and $u$ by virtue of (2.1.12) and Theorem 2.1.2'. Therefore we can apply Proposition 4.2.4 of S-K-K [2] Chapter II to $T\left(D^{+}\right)$, and conclude that $T\left(D^{+}\right)$, hence (the connected part of) the $S$-matrix, must satisfy a simple holonomic system of micro-differential equations whose characteristic variety is given by $\mathcal{L}^{c}(D)$. Its order is calculated in the same way as in the proof of Theorem 2.1.1.

Q.E.D.

We complete this subsection by showing how to extract out the "positive- $\alpha$ part" $\left[T\left(D^{+}\right)\right]_{+\alpha}$ from $T\left(D^{+}\right)$in the situation discussed in this section. More precisely, we show that the restriction of $T\left(D^{+}\right)$ to a real neighborhood $\omega$ of $\left(p_{0} ; \sqrt{-1} u_{0}\right)$ coincides with $\left[T\left(D^{+}\right)\right]_{+\alpha}$, as microfunctions.

\section{Theorem 2.1.4.}

Assume that $\left(p_{0} ; \sqrt{-1} u_{0}\right)$ belongs to $\mathcal{L}_{0}\left(D^{+}\right)$for a unique positive$\alpha$ Landau diagram $D^{+}$, which is simple and connected, and that $p_{0}$ is an elementary point with respect to $D^{+}$. Then for some sufficiently small neighborhood $\omega$ of $\left(p_{0} ; \sqrt{-1} u_{0}\right)$ in $\sqrt{-1} S^{*} \mathcal{M}_{r}$ and some sufficiently small positive values of the constants $\varepsilon_{l}$ and $\varepsilon_{l}^{\prime}(l=1, \cdots, N)$ there is for each $j$ a collection of off-mass-shell extensions of $s_{j}(p, k)$ such that the integral $\left[T\left(D^{+}\right)\right]_{+\alpha}$ defined by (2.1.14) below is well defined in $\omega$ and satisfies

$$
\left[T\left(D^{+}\right)\right]_{+\alpha}=T\left(D^{+}\right) \text {microlocally in } \omega .
$$

The function $\left[T\left(D^{+}\right)\right]_{+\alpha}$ is defined by

$$
\frac{1}{(2 \pi i)^{N}} \int \prod_{j=1}^{n^{\prime}} s_{j}(p, k) \delta^{4}\left(\sum_{r=1}^{n}[j: r] p_{r}+\sum_{l=1}^{N}[j: l] k_{l}\right) \times
$$




$$
\times \prod_{l=1}^{N} \frac{Y\left(k_{l, 0}\right) Y\left(k_{l}{ }^{2}-m_{l}{ }^{2}+\varepsilon_{l}{ }^{\prime}\right) Y\left(m_{l}{ }^{2}+\varepsilon_{l}-k_{l}{ }^{2}\right)}{k_{l}{ }^{2}-m_{l}{ }^{2}+i 0} \prod_{l=1}^{N} d^{4} k_{l}
$$

Proof.

Let $W$ be a neighborhood of $p$ and define

$$
\begin{aligned}
& G=\left\{(p, k) ; p \in \bar{W}, \sum_{r=1}^{n}[j: r] p_{r}+\sum_{l=1}^{N}[j: l] k_{l}=0 \quad\left(j=1, \cdots, n^{\prime}\right),\right. \\
& p_{r}{ }^{2}=\mu_{r}{ }^{2} \text { and } p_{r, 0}>0 \quad(r=1, \cdots, n), \\
& \left.-\varepsilon_{l}{ }^{\prime} \leqq k_{l}{ }^{2}-m_{l}{ }^{2} \leqq \varepsilon_{l} \text { and } k_{l, 0}>0 \quad(l=1, \cdots, N)\right\} .
\end{aligned}
$$

Then the projection map from $(p, k)$ to $p$ is proper (i.e., the inverse image of a compact set is compact) when it is restricted to $G$, since the diagram $D$ is partially ordered. In fact, if we cut the diagram $D$ by any vertical line $\lambda$ that does not intersect any vertex then the sum of the energies associated with the lines cut by $\lambda$ is bounded, if $p$ is confined to a bounded set. Hence $\vec{k}_{l}$, the vector part of $k_{l}$, must be bounded in $G$, because $\sqrt{{\overrightarrow{k_{l}}}^{2}+m_{l}{ }^{2}-\varepsilon_{l}{ }^{\prime}} \leqq k_{l, 0} \leqq \sqrt{{\overrightarrow{k_{l}}}^{2}+m_{l}{ }^{2}+\varepsilon_{l}}$. Therefore by the imbedding theorem of Grauert [20], we can find some off-shell extensions $s_{j}(p, k)$ that are defined in a neighborhood of $G$ for sufficiently small $\varepsilon_{l}>0, \varepsilon_{l}^{\prime}>0$ and $W$.

For each $j$ we now choose any fixed one of these off-mass-shell extensions $s_{j}(p, k)$, and prove the following lemma.

\section{Lemma 2.1. 5 .}

The integral $[T(D)]_{0}$ defined by (2.1.15) below is a well-defined hyperfunction and its singularity spectrum is contained in the set defined by (2.1.16). Here $\sigma=\left(\sigma_{1}, \cdots, \sigma_{N}\right)$ and each $\sigma_{l}$ is either +1 or -1 . The function $[T(D)]_{\sigma}$ is defined by

$$
\begin{gathered}
{[T(D)]_{\sigma}=\frac{(-1)^{n(\sigma)}}{(2 \pi i)^{N}} \int \prod_{j=1}^{n^{\prime}} s_{j}(p, k) \delta^{4}\left(\sum_{r=1}^{n}[j: r] p_{r}+\sum_{l=1}^{N}[j: l] k_{l}\right) \times} \\
\quad \times \prod_{l=1}^{N} \frac{Y\left(k_{l, 0}\right) Y\left(k_{l}{ }^{2}-m_{l}{ }^{2}+\varepsilon_{l}{ }^{\prime}\right) Y\left(m_{l}{ }^{2}+\varepsilon_{l}-k_{l}{ }^{2}\right)}{k_{l}{ }^{2}-m_{l}{ }^{2}+i \sigma_{l} 0} \prod_{l=1}^{N} d^{4} k_{l},
\end{gathered}
$$

where $n(\sigma)=\#\left\{l ; \sigma_{l}=-1\right\}$, and its singularity spectrum is confined to the set $\left\{(p ; \sqrt{-1} u) \in \sqrt{-1} S^{*} \mathscr{M}\right.$; there exist $\alpha_{l}, \beta_{r}, v_{j}$ and $k_{l}$ which 
satisfy (2.1.16) below.\}

(2. 1. 16)

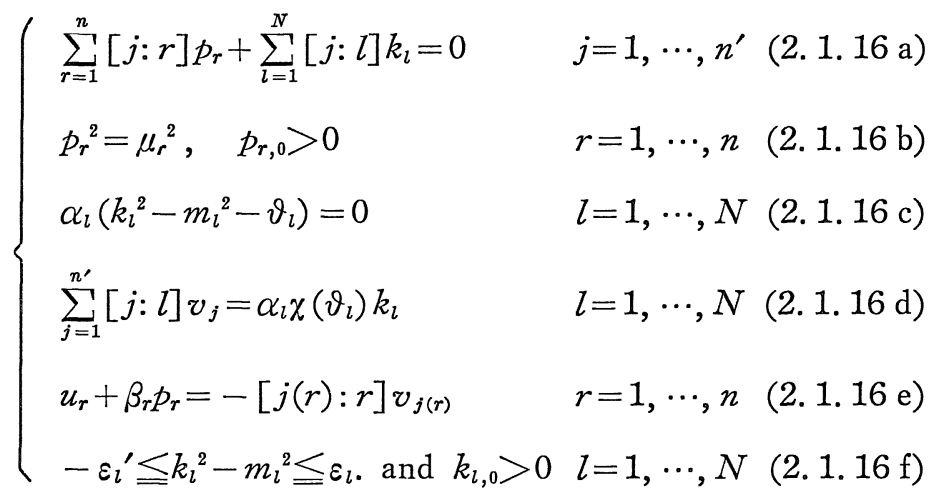

Here $\vartheta_{l}$ is allowed to be either $\varepsilon_{l}, \varepsilon_{l}{ }^{\prime}$, or 0 and

$$
\chi\left(\vartheta_{l}\right)= \begin{cases}\sigma_{l} & \text { if } \vartheta_{l}=0 \\ +1 \text { or }-1 & \text { if } \vartheta_{l}=\varepsilon_{l} \text { or } \varepsilon_{l}{ }^{\prime}\end{cases}
$$

Proof of Lemma 2.1. 5.

First recall that

S.S. $\delta(\phi(x))=\left\{(x ; \sqrt{-1} \eta) \in \sqrt{-1} S^{*} \mathcal{M} ;\right.$

$$
\left.\phi(x)=0, \eta= \pm \operatorname{grad}_{x} \phi(x)\right\}
$$

$$
\text { S.S. } Y(\phi(x))=\left\{(x ; \sqrt{-1} \eta) \in \sqrt{-1} S^{*} \mathscr{M} ;\right.
$$

$$
\left.\phi(x)=0, \eta= \pm \operatorname{grad}_{x} \phi(x)\right\}
$$

$$
\begin{gathered}
\text { S.S. }\left(\frac{1}{\phi(x) \pm i 0}\right)=\left\{(x ; \sqrt{-1} \eta) \in \sqrt{-1} S^{*} \mathcal{M} ;\right. \\
\left.\phi(x)=0, \eta= \pm \operatorname{grad}_{x} \phi(x)\right\}
\end{gathered}
$$

hold if $\phi(x)$ is a real-valued real analytic function defined on $M$ with the property that $\operatorname{grad}_{x} \phi(x)$ never vanishes on $\{x \in M ; \phi(x)=0\}$.

Therefore, applying Theorem 2.4.1 of S-K-K [2] Chapter I to the integrand of $[T(D)]_{\sigma}$, we find that its singularity spectrum is confined to the set defined below:

$\left\{(p, k ; \sqrt{-1}(u, w))\right.$; there exist $\alpha_{l}, \beta_{r}$ and $v_{j}$ which satisfy (2.1.19) below\}

$$
\left(\sum_{r=1}^{n}[j: r] p_{r}+\sum_{l=1}^{N}[j: l] k_{l}=0 \quad j=1, \cdots, n^{\prime}\right.
$$




$$
\text { (2.1.19) }\left\{\begin{array}{lll}
p_{r}{ }^{2}=\mu_{r}{ }^{2}, p_{r, 0}>0 & r=1, \cdots, n & (2.1 .19 \mathrm{~b}) \\
\alpha_{l}\left(k_{l}{ }^{2}-m_{l}{ }^{2}-\vartheta_{l}\right)=0 & l=1, \cdots, N & (2.1 .19 \mathrm{c}) \\
\sum_{j=1}^{n^{\prime}}[j: l] v_{j}-\alpha_{l} \chi\left(\vartheta_{l}\right) k_{l}=w_{l} & l=1, \cdots, N & (2.1 .19 \mathrm{~d}) \\
u_{r}+\beta_{r} p_{r}=-[j(r): r] v_{j(r)} & r=1, \cdots, n & (2.1 .19 \mathrm{e}) \\
-\varepsilon_{l}{ }^{\prime} \leqq k_{l}{ }^{2}-m_{l} \leqq \varepsilon_{l}, k_{l, 0}>0 & l=1, \cdots, N & (2.1 .19 \mathrm{f})
\end{array}\right.
$$

Here we have used the Corollary of Theorem 0.0.

On the other hand, the integral $[T(D)]_{\sigma}$ is a proper integral (as an integral of a hyperfunction) in the sense that the support of its integrand $G$ is compact as long as $p$ is confined to a compact set.

Therefore Theorem 2.3.1 of S-K-K [2] Chapter I is immediately applicable to our case and we obtain (2.1.16). This completes the proof of Lemma 2.1.5.

\section{Proof of Theorem 2.1.4 continued.}

Since $\delta\left(k_{l}{ }^{2}-m_{l}{ }^{2}\right)=\frac{1}{2 \pi i}\left(\frac{1}{k_{l}{ }^{2}-m_{l}{ }^{2}+i 0}-\frac{1}{k_{l}{ }^{2}-m_{l}{ }^{2}-i 0}\right)$ holds, and since $[T(D)]_{\sigma}$ is a well-defined hyperfunction for any $\sigma, T\left(D^{+}\right)$is equal to $\sum_{\sigma}[T(D)]_{\sigma}$. Hence Lemma 2.1.5 implies that $[T(D)]_{\sigma}=0$ (as a microfunction) in a neighborhood $\omega$ of $\left(p_{0} ; \sqrt{-1} u_{0}\right)$ unless $\sigma=(+1, \cdots$, $+1)$. Here we have used the following two facts:

First, for a sufficiently small neighborhood $\omega$ of $\left(p_{0} ; \sqrt{-1} u_{0}\right)$, $\mathcal{L}\left(D^{\sigma}\left(m_{l}{ }^{2}+\delta_{l}\right)\right) \cap \omega=\phi$ if $\sigma \neq(+1, \cdots,+1)$. Here $D^{\sigma}\left(m_{l}{ }^{2}+\delta_{l}\right)$ denotes the same Landau diagram as the original $D^{+}$except that the masses associated with $L_{l}$ is $m_{l}{ }^{2}+\delta_{l}$ and the signs $\sigma_{l}$ are given by $\sigma$. In fact, Theorem 0.0 says that $(p ; \sqrt{-1} u)$ does not belong to $\mathcal{L}\left(D^{\sigma}\right)$, for $\sigma \neq(+1, \cdots,+1)$, hence, by the continuity of $\mathcal{L}\left(D^{\sigma}\left(m_{l}{ }^{2}+\delta_{l}\right)\right)$ with respect to $\delta_{l}$, we can find such a neighborhood $\omega$.

Secondly, we claim that $\mathcal{L}\left(D^{+}\left(m_{l}{ }^{2}+\delta_{l}\right)\right) \cap \omega=\phi$ if some $\delta_{l} \neq 0$, for sufficiently small $\omega$. In fact, as in the proof of Theorem 2.1.3, we can find $D_{j}{ }^{+}\left(j=1, \cdots, j_{0}\right)$ such that $p_{0} \in \bigcap_{j=1}^{j_{0}} L_{0}\left(D_{j}^{+}\right)$. Recall the fact that $L_{0}\left(D_{j}^{+}\right)$defines a nonsingular hypersurface in $\mathcal{M}_{r}$ (Chandler-Stapp [7] Theorem 6). Furthermore, the argument there also shows that $L_{0}\left(D_{j}{ }^{+}\left(m_{l}{ }^{2}\right)\right)$ defines a nonsingular hypersurface of $\mathcal{M}_{r} \times\left\{\left(m_{1}{ }^{2}, \cdots, m_{N_{j}}^{2}\right) \in \boldsymbol{R}^{N_{j}}\right\} \quad\left(N_{j} \equiv\right.$ 
$\equiv \#$ (internal lines of $\left.D_{j}\right)$ ) and that we can choose a defining function $\phi_{j}\left(p, m_{l}{ }^{2}\right)$ of this hypersurface so that

$$
\left(\frac{\partial \phi_{j}}{\partial p_{r}}, \frac{\partial \phi_{j}}{\partial m_{l}^{2}}\right)_{\substack{r=1, \ldots, n \\ l=1, \cdots, N_{j}}}=c\left(u_{r}, \alpha_{l}\right)_{\substack{r=1, \cdots, n \\ l=1, \cdots, N_{j}}}(c>0)
$$

Therefore $L_{0}\left(D_{j}{ }^{+}\left(m_{l}{ }^{2}\right)\right) \cap L_{0}\left(D_{j}{ }^{+}\left(m_{l}{ }^{2}\right)\right)=\phi$ if $m_{l} \neq m_{l}{ }^{\prime}$ and the internal line $L_{l}$ corresponds to an internal line of $D_{j}$. On the other hand, each internal $L_{l}$ of $D^{+}$corresponds to some internal line of some diagram $D_{j}{ }^{+}$. This follows from the construction of Chandler-Stapp [7], which expresses the $\alpha^{\prime}$ s of $D^{+}$as positive linear combinations of the $\alpha^{\prime}$ s of the $D_{j}{ }^{+}$, and the fact that $(p ; \sqrt{-1} u)$ lies on $\mathcal{L}_{0}\left(D^{+}\right)$, which implies all $\alpha_{l}>0$. This proves the second assertion above.

Thus we have verified that $[T(D)]_{+\alpha}=T\left(D^{+}\right)$microlocally near $\left(p_{0} ; \sqrt{-1} u_{0}\right)$ for sufficiently small $\varepsilon_{l}, \varepsilon_{l}{ }^{\prime}>0$ and certain off-shell extension of $s_{j}(p, k)$. This completes the proof of the theorem.

\section{§2.2. Study of the holonomic structure near points where several simple Landau diagrams are relevant}

In $\S 2.1$ we investigated the holonomic structure near points where only one diagram $D^{+}$is relevant. In that case, the point $(p ; \sqrt{-1} u)$ in question belongs to only one Landau variety, and the characteristic variety of the holonomic system that the $S$-matrix satisfies is nonsingular there. However, to understand fully the holonomic structure of the $S$-matrix it is necessary to investigate it also near points where the irreducible components of the characteristic varieties associated with several different Landau diagrams meet. The following theorem covers cases of this kind in which all the relevant diagrams are simple, and some are contractions of others.

Theorem 2.2.1.

Let $\left(p_{0} ; \sqrt{-1} u_{0}\right)$ be a point in $\sqrt{-1} S^{*}\left(\mathcal{M}-\mathcal{M}_{0}\right)$. Let $D$ be a simple connected Landau diagram. Suppose the following conditions are satisfied:

$$
\left(p_{0} ; \sqrt{-1} u_{0}\right) \text { lies on } \mathcal{L}_{2}\left(D^{+}\right) \text {. }
$$


(2.2.2) For each vertex $b$ of $D$ the vector $p^{b}$ defined in (2.1.0) satisfies one of the following two conditions:

(a) $p^{b} \notin L^{+}$

(b) The following three conditions are satisfied.

(i) There is a unique $D_{b}$ such that $p^{b}$ lies on $L_{0}\left(D_{b}\right)$.

(ii) This $D_{b}$ is simple and connected.

(iii) $p^{b} \in L_{e}\left(D_{b}^{+}\right)$.

(2.2.3) There is no nontrivial solution $(p ; u)=\left(p_{0} ; 0\right)$ of the complexified Landau equations associated with any $D_{i}$ that fits into $T\left(D^{+}\right)$and satisfies $\left(p_{0} ; \sqrt{-1} u_{0}\right) \in \mathcal{L}\left(D_{i}^{+}\right)$.

(2.2.4) There is a complex neighborhood $\omega$ of $\left(p_{0} ; \sqrt{-1} u_{0}\right)$ such that for each $(p ; u) \in \omega$ and each internal line $L_{l}$ of $D$ the set of values of $\alpha_{l}$ allowed by the complexified Landau equations associated with $T\left(D^{+}\right)$consists of at most a single point in $\mathbb{C}^{1}$. Morever the allowed value of $\alpha_{l}$ ranges over a compact set as $(p ; u)$ ranges over a compact set.

Then the S-matrix $S(p)$ satisfies a holonomic system $\mathfrak{M}$ of microdifferential equations in a neighborhood of $\left(p_{0} ; \sqrt{-1} u_{0}\right)$. Furthermore, the characteristic variety of $\mathfrak{M}$ is given by the union of the complexified Landau equations associated with the diagrams $D_{i}$ specified in (2.2.3).

Proof.

The microlocal discontintuity formula and conditions (2.2.1), (2.2.2) and (2.2.3) entail that the $S$-matrix $S(p)$ be given in a neighborhood of $\left(p_{0} ; \sqrt{-1} u_{0}\right)$ (as a microfunction, and apart from constant factors) by

$$
\int \prod_{b} S^{b}(p, k) \prod_{l=1}^{N} \delta^{+}\left(k_{l}{ }^{2}-m_{l}{ }^{2}\right) d^{4} k_{l},
$$

where $b$ runs over the boxes of $T\left(D^{+}\right)$(or vertices of $D$ ), and $k$ denotes the set of energy-momentum four-vectors associated with the explicit internal lines of $T\left(D^{+}\right)$. Furthermore, by condition (2.2.2) each $S^{b}$ is evaluated at a regular point or at a point of $L_{e}\left(D_{b}\right)$.

Now we apply Corollary 1 of Theorem 2.1.1 to $S^{b}(p)$. Then the integral (2.2.6) can be rewritten in the following form 
(2. 2.7)

$$
\begin{aligned}
\int \prod_{b} & \left(h_{1}{ }^{b}(p, k) \Phi_{\lambda_{b}}\left(\phi_{b}(p, k)+i 0\right)+h_{2}{ }^{b}(p, k)\right) \times \\
& \times \prod_{b} \delta^{4}\left(\sum_{r}[b: r] p_{r}+\sum_{l}[b: l] k_{l}\right) \prod_{l=1}^{N} \delta^{+}\left(k_{l}{ }^{2}-m_{l}{ }^{2}\right) d^{4} k_{l},
\end{aligned}
$$

where

$$
\Phi_{\lambda}(\tau)=\left\{\begin{array}{l}
\frac{(-1)^{\lambda+1} \Gamma(\lambda+1)}{\tau^{\lambda+1}} \text { if } \lambda \text { is not a negative integer } \\
\frac{(-1)^{\lambda+1}}{(-\lambda-1) !} \tau^{-\lambda-1}\left\{\log \tau-\sum_{\nu=1}^{-\lambda-1} \frac{1}{\nu}-\gamma\right\} \\
\text { if } \lambda \text { is a negative integer ( } \gamma: \text { Euler constant })
\end{array}\right.
$$

and

$$
\lambda_{b}=\alpha\left(D_{b}\right)-\frac{5}{2}
$$

We next seek the holonomic system that the integrand should satisfy.

First define $b^{ \pm}(l)$ as the unique box $b$ such that the incidence number $\left[b^{ \pm}(l): l\right]= \pm 1$. And define $b(r)$ as the unique box $b$ such that $[b(r): r] \neq 0$. Then Theorem 3.5.3 of S-K-K [2] Chapter II guarantees the existence of a holonomic system satisfied by the integrand provided the following equation (2.2.8) does not admit a solution $(p, k ; u, \zeta)$ $=(p, k ; 0,0)$ for any sets of four-vectors $u^{(b)}=\left(u_{1}{ }^{(b)}, \cdots, u_{n}{ }^{(b)}\right), w^{(b)}=$ $=\left(w_{1}{ }^{(b)}, \cdots, w_{N}{ }^{(b)}\right)$ and complex scalars $c_{b}$. Here $b$ runs over the boxes of $T\left(D^{+}\right), n$ denotes the number of the external lines of $D$, and $N$ denotes the number of internal lines of $D$.

$$
\begin{array}{ll}
u_{r}=c_{b(r)} u_{r}^{(b(r))} & r=1, \cdots, n \\
-c_{b-(l)} w_{l}^{(b-(l))}+c_{b+(l)} w_{l}^{\left(b^{+}(l)\right)}=\zeta_{l} \quad l=1, \cdots, N \\
\left(p_{r}, k_{l} ; u^{(b)}, w^{(b)}\right) \text { with every } r \text { such that }[b: r] \neq 0 \text { and every } \\
l \text { such that }[b: l] \neq 0 \text { satisfies either }
\end{array}
$$

* Since $u_{r}^{(b)}\left(w_{l}{ }^{(b)}\right.$, resp.) is zero if $[b: r]=0\left([b: l]=0\right.$, resp.) such components of $u^{(b)}$ and $w^{(b)}$ are disregarded in the above. 


$$
\begin{aligned}
& \text { or } \\
& \left(u_{r}^{(b)}, w_{l}^{(b)}\right)=\left(a^{(b)}, a^{(b)}\right)
\end{aligned}
$$

with some four-vector $a^{(b)}$, for $r$ with $[b: r] \neq 0$ and $l$ with $[b: l] \neq 0$, according to whether $S_{b}$ is evaluated at a point of $L_{e}\left(D_{b}\right)$ or at a regular point.

On the other hand, $\left\{\left(p_{r}, k_{l} ; u_{r}{ }^{(b)}, w_{l}{ }^{(b)}\right)\right.$ with $[b: r] \neq 0$ and $[b: l] \neq 0$; $\left.\phi_{b}(p, k)=0, \quad\left(u_{r}^{(b)}, w_{l}^{(b)}\right)=c \operatorname{grad}_{\left(p_{r}, k_{l}\right)} \phi_{b}(p, k), c>0\right\} \quad$ coincides with $\mathcal{L}_{0}\left(D_{b}{ }^{+}\right)$by virtue of the choice of $\phi_{b}(p, k)$. Therefore, replacing $\left(u^{(b)}, w^{(b)}\right)$ by $\left(c_{b} u^{(b)}, c_{b} w^{(b)}\right)$, the intersection of variety defined by (2.2.8) and $\left\{(p, k ; u, \zeta) ; \zeta_{l}=0, l=1, \cdots, N\right\}$ defines the Landau variety associated with a $D_{1}$ that fits into $T\left(D^{+}\right)$. (See the Landau equation given in the form of $(0.4))$. Therefore for all $(p ; u)$ in some sufficiently small neighborhood of $\left(p_{0} ; u_{0}\right)$, assumption (2.2.3) guarantees that there is no solution of (2.2.8) of the form $(p, k ; u, \zeta)=(p, k ; 0,0)$.

Then the same Theorem 3.5.3 of S-K-K [2] asserts that the integrand of (2.2.7) satisfies a holonomic system of micro-differential equations whose characteristic variety is given by (2.2.8).

On the other hand, Theorem 3.5.5 of S-K-K [2] Chapter II guarantees that the integral given by (2.2.7) satisfies a holonomic system if the equation (2.2.8) supplemented by $\zeta_{l}=0(l=1, \cdots, N)$ determines $k_{l}(l=1, \cdots, N), u^{(b)}$ and $w^{(b)}$ uniquely by $\left(p_{r} ; u_{r}\right)$, and they are confined to a compact set as long as $\left(p_{r} ; u_{r}\right)$ runs over a compact set. Furthermore, Theorem 3.5.5 asserts its characteristic variety is given by setting $\zeta_{l}=0(l=1, \cdots, N)$ in (2.2.8). As noticed earlier, the variety thus obtained is precisely the Landau variety. Hence it suffices to show the determinancy and boundedness of $u^{(b)}$ and $w^{(b)}$.

As discussed earlier (cf. the last part of the proof of Theorem 2.1.4) $k_{l}$ 's are determined uniquely by $p$ and bounded. On the other hand, the $\alpha_{l}$ 's associated with the internal lines of $D$ are also determined uniquely by $(p ; u)$ and are bounded by assumption (2.2.4). Since the Landau constants associated with the internal lines of $D_{b}$ are uniquely determined by $p$ and $k$, this assumption entails the required uniqueness and boundedness assertion on $\left(u^{(b)}, w^{(b)}\right)$. This completes the proof of Theorem 2.2.1. 
To understand the relationship of Theorem 2.2.1 with the "hierarchical principle" (Eden et al. [24]), we shall discuss two simple cases in a little more detail. More complete arguments can be found in Kashiwara-Kawai-Oshima [22].

The first case is that in which only two diagrams are relevant, both are simple, and the second is obtained by contracting a single line of the first.

\section{Example 1.}

Suppose all the conditions required in Theorem 2.2.1 are satisfied, and condition (2.2.2 a) is satisfied for all $b$ except one. Suppose this one $b$ satisfies $\left(2.2 .2 \mathrm{~b}\right.$ ) with a (pole) diagram $D_{b}$ that has precisely one internal line. Let $D_{1}$ be the diagram constructed by inserting this $D_{b}$ for the corresponding vertex $b$ of $D \equiv D_{2}$. Assume that $\overline{\mathcal{L}}_{0}\left(D_{1}\right)$ and $\mathcal{L}_{0}\left(D_{2}\right)$ intersect transversally at $\left(p_{0} ; u_{0}\right)$. Assume further that the following condition is satisfied:

Let $f_{i}(p, u, k, v, \alpha, \beta), i=2, \cdots, i(D) \equiv 4\left(n+N+n^{\prime}\right)+n^{\prime}+N$, be the set of the defining equations of the Landau variety (0.1) other than $k_{1}{ }^{2}=m_{1}{ }^{2}$. Let $g_{1}$ be $k_{1}{ }^{2}-m_{1}{ }^{2}$ and let $h_{1}$ be $\alpha_{1}$. Assume that the following conditions are satisfied:

$$
\begin{aligned}
& \operatorname{dim} \mathcal{L}\left(D_{1}\right), \operatorname{dim} \mathcal{L}\left(D_{2}\right) \geqq 4 n-1 \\
& \operatorname{rank}\left(\frac{\partial\left(g_{1}, h_{1}, f_{2}, \cdots, f_{i(D)}\right)}{\partial(p, u, k, v, \alpha, \beta)}\right)=i(D)+1 \\
& \operatorname{rank}\left(\frac{\partial\left(g_{1}, f_{2}, \cdots, f_{i(D)}\right)}{\partial(p, u, k, v, \alpha, \beta)}\right)=i(D) \\
& \operatorname{rank}\left(\frac{\partial\left(h_{1}, f_{2}, \cdots, f_{i(D)}\right)}{\partial(p, u, k, v, \alpha, \beta)}\right)=i(D)
\end{aligned}
$$

Then the holonomic system $\mathfrak{M}$ derived in Theorem 2.2.1 is simple in the sense that its symbol ideal is reduced. (See Kashiwara-Kawai-Oshima [22].) Furthermore, its order on $\mathcal{L}_{0}\left(D_{1}\right)$ is given by $\alpha\left(D_{1}\right)$ and that on $\mathcal{L}_{0}\left(D_{2}\right)$ is given by $\alpha\left(D_{2}\right)\left(=\alpha\left(D_{1}\right)-\frac{1}{2}\right)$.

Note that all the conditions above are satisfied if at least two external lines are attached to each vertex of $D_{1}$. Note also that the order 
of the holonomic system that the scattering amplitude satisfies is decreased by 2 as a result of factorizing out the over-all $\delta$-function.

An interesting subcase is the case where $\pi\left(\mathcal{L}\left(D_{1}\right)\right)$ and $\pi\left(\mathcal{L}\left(D_{2}\right)\right)$ define real nonsingular hypersurfaces $H_{1}$ and $H_{2}$, respectively (in the restricted mass-shell manifold). In this case, the transversality of $\mathcal{L}\left(D_{1}\right)$ and $\mathcal{L}\left(D_{2}\right)$ entail that $H_{1}$ and $H_{2}$ are tangent to each other exactly to the second order along $H_{1} \cap H_{2}$. Thus we can find a local coordinate system $(x)=\left(x_{1}, \cdots, x_{m}\right)$ in a neighborhood of $p_{0}$ so that $H_{1}$ is given by $\left\{x ; h_{1}(x)=x_{1}-x_{2}{ }^{2}=0\right\}$ and $H_{2}$ is given by $\left\{x ; h_{2}(x)=x_{\text {def. }}=0\right\}$. Then a result of Kashiwara-Kawai-Oshima [22] tells us that a system that is simple and whose characteristic variety is confined to $\mathcal{L}\left(D_{1}\right) \cup \mathcal{L}\left(D_{2}\right)$ can be brought to the following canonical form under a suitable "quantized" contact transformation:

$$
\mathfrak{M}_{D_{1}, D_{2}}:\left\{\begin{array}{l}
\left(x_{1} D_{x_{1}}+\frac{1}{2} x_{2} D_{x_{2}}+\lambda\right) f=0 \\
\left(\left(x_{2} D_{x_{1}}+\frac{1}{2} D_{x_{2}}\right) D_{x_{2}}+2 \alpha D_{x_{1}}\right) f=0 \\
\frac{\partial}{\partial x_{3}} f=\cdots=\frac{\partial}{\partial x_{n}} f=0,
\end{array}\right.
$$

where

$$
\lambda=\frac{1}{2}\left(\alpha\left(D_{1}\right)-2+\alpha\left(D_{2}\right)-2+\frac{3}{2}\right)=\alpha\left(D_{1}\right)-\frac{3}{2}
$$

and

$$
\alpha=\frac{1}{2}\left(\left(\alpha\left(D_{1}\right)-2\right)-\left(\alpha\left(D_{2}\right)-2\right)+\frac{1}{2}\right)=\frac{1}{2} .
$$

Hence, after some calculation (Kashiwara-Kawai-Oshima [22]), one can conclude the scattering amplitude $s(p)$ regarded as a microfunction has the following form (2.2.13) in this coordinate system:

$$
\varphi_{1}(x) f(\alpha, \lambda ; x)+\varphi_{2}(x) f\left(\alpha+\frac{1}{2}, \lambda-\frac{1}{2} ; x\right)
$$

where $\varphi_{1}(x)$ and $\varphi_{2}(x)$ are analytic and $f(\alpha, \lambda, x)=$

$$
=\frac{\Gamma(\lambda)}{2 \Gamma\left(\alpha+\frac{1}{2}\right)}\left(x_{1}+i 0\right)^{-\lambda} F\left(\lambda, \alpha, \frac{1}{2} ; \frac{x_{2}{ }^{2}}{x_{1}+i 0}\right)+
$$




$$
+\frac{\Gamma\left(\lambda+\frac{1}{2}\right)}{\Gamma(\alpha)} x_{2}\left(x_{1}+i 0\right)^{-\lambda-\frac{1}{2}} F\left(\lambda+\frac{1}{2}, \alpha+\frac{1}{2}, \frac{3}{2} ; \frac{x_{2}^{2}}{x_{1}+i 0}\right) .
$$

Here $F(\alpha, \beta, \gamma ; z)$ stands for hypergeometric series.

By making use of this concrete expression, one can easily conclude that the scattering amplitude is locally square summable if $\alpha\left(D_{1}\right)-\frac{3}{2} \leqq 0$, i.e., if $\frac{3}{2} N-2 n^{\prime}+\frac{3}{2} \geqq 0$, and hence one can define the product of the scattering amplitude $s(p)$ and its complex conjugate $s(p)^{\dagger}$ in a neighborhood of $p_{0}$. In this case, the singularity spectrum of the resulting function is confined to $\sqrt{-1} S^{*}{ }_{H_{1}} \mathscr{M}_{r} \cup \sqrt{-1} S^{*}{ }_{H_{2}} \mathscr{M}_{r} \cup \sqrt{-1} S_{H_{1} \cap H_{2}}^{*} \mathscr{M}_{r}$. Here one encounters an extra singularity at $H_{1} \cap H_{2}$, which is not described by the ordinary Landau equations. This observation will lead us naturally to extend the notion of Landau variety when we are dealing with mixed- $\alpha$ singularities. This is one of our motivations for introducing the notion of the extended Landau variety $\widetilde{\mathcal{L}}(D)$. (See Kashiwara-Kawai-Stapp [6] for the definition of the extended Landau varieties $\widetilde{L}(D)$.)

In Example 1, the holonomic system involved is simple. However, this is a rather exceptional case and, in general, we cannot expect its simplicity. This is closely tied to the following fact: if some $S^{b}$ is evaluated at $L_{0}\left(D_{b}\right)$, and if $\alpha\left(D_{b}\right) \neq 5 / 2$, then $L_{0}\left(D_{b}\right)$ is a branch point (not a pole) of $s^{b}$, the scattering function obtained from $S^{b}$ by factorizing out the over-all $\delta$-function.

To explain the situation in a more concrete way we restrict ourselves to the case in which $D_{1}^{+}$is 'external' in order to avoid technical complexities.

\section{Example 2.}

Assume all the conditions of Example 1 except that now the unique $D_{b}$ is a simple diagram with one closed loop, and that $D \equiv D_{2}$ is obtained by contracting out this closed loop $D_{b}$ of $D_{1}$. Assume further that at least two nonparallel external lines are attached to each vertex of $D_{1}$, as in the diagrams below: 

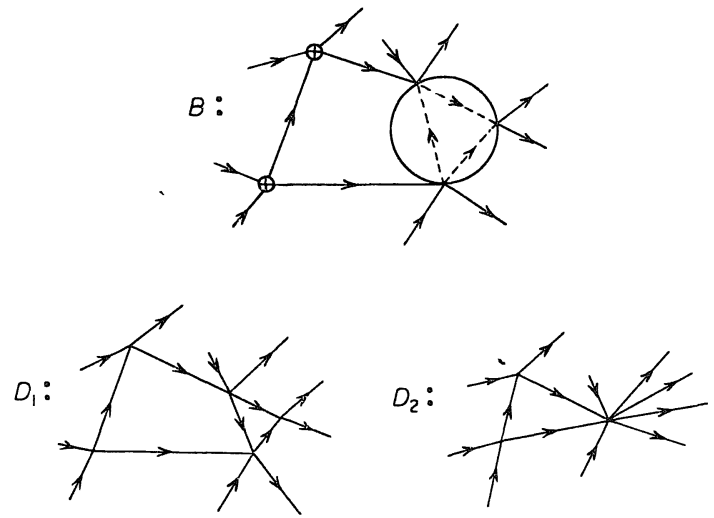

Assume further that $\alpha\left(D_{b}\right) \neq 5 / 2$ (hence $\alpha\left(D_{b}\right)<5 / 2$ by (2.1.10)).

In this case the same analysis as in Example 1 shows the following:

The scattering amplitude $s(p)$ is a sum of two functions $f_{1}(p)$ and $f_{2}(p)$ which satisfy the following:

$f_{1}(p)$ satisfies a simple holonomic system $\mathfrak{M}_{1}$ whose characteristic variety is confined to $\mathcal{L}\left(D_{1}\right) \cup \mathcal{L}\left(D_{2}\right)$, while $f_{2}(p)$ satisfies a simple holonomic system $\mathfrak{M}_{2}$ whose characteristic variety is confined to $\mathcal{L}\left(D_{2}\right)$. Furthermore, the order of $\mathfrak{M}_{1}$ on $\mathcal{L}_{0}\left(D_{1}\right)$ is $\alpha\left(D_{1}\right)-2$ and that on $\mathcal{L}_{0}\left(D_{2}\right)$ is $\alpha\left(D_{2}\right)-2$. However, $\alpha\left(D_{2}\right) \neq \alpha\left(D_{1}\right)-\frac{1}{2}$ in this case. In fact, $\alpha\left(D_{1}\right)$ $=\alpha\left(D_{2}\right)+\left(\alpha\left(D_{b}\right)-2\right)$. In this case it is known (Kashiwara-KawaiOshima [22]) that there is no micro-differential operator $Q\left(p, D_{p}\right)$ that sends $f_{1}(p)$ to $f_{2}(p)$. Such an operator $Q$ does exist in the case of Example 1, and this is the reason why simplicity holds in that case.

We should, however, emphasize that this result does not necessarily mean that there is no relation between $f_{1}$ and $f_{2}$. It is highly probable that the unitarity relation entails some functional relationship between $f_{1}$ and $f_{2}$ that cannot be described by linear micro-differential equations. At least, the result of Zimmermann [23] on the two-particle threshold singularity structure (cf. §3.1) suggests that such a connection should exist.

\section{$\S 3$. Verification of Sato's conjecture for diagrams with at most double lines}

In this section the results of $\S 2$ are extended to the case where 
the relevant diagrams contain at most double lines (i.e., at most two lines connect any pair of vertices). The arguments rely on the square-root character of the singularities at two-particle threshold points. The squareroot character of these singularities are derived in the first subsection from the general $S$-matrix discontinuity formula by employing the methods used by Zimmermann [23] to study the leading normal-threshold singularity in the 2 to 2 case.

\section{§.1. Singularity structure of the $S$-matrix at two-particle threshold points}

In this section we derive the square-root character of the $S$-matrix at two-particle threshold points.

\section{Definition 3. 1. 1 .}

A two-particle threshold point is a value $\bar{p}$ of the argument of a scattering function $s(p)$ that satisfies the following five conditions:

(a) Let $\left\{D_{j} ; j \in J\right\}$ be the set of unsigned diagrams such that $\bar{p} \in \bar{L}_{0}\left(D_{j}{ }^{+}\right)$. Then every $D_{j}{ }^{+}$in this set is a two-particle normal threshold diagram, which is a diagram with exactly two internal lines and exactly two vertices.

(b) For each $j$ in $J$ there is a pair of distinct vectors $\left(p_{r^{(j)}}, p_{r^{\prime}(j)}\right)$, taken from the set $\left(p_{1}, \cdots, p_{n}\right) \equiv p$, such that $\varepsilon_{r(j)}=\varepsilon_{r^{\prime}(j)}$ and the surface $L_{0}\left(D_{j}^{+}\right)$consists of the points $p \in \mathcal{M}_{r}$ such that $\bar{p}_{r(j)}$ is parallel to $\bar{p}_{r^{\circ}(j)}$.

(c) For every sufficiently small complex neighborhood $\widetilde{\omega}$ in the space of Lorentz scalars such that its inverse image $\omega$ in $\mathscr{M}_{r} \boldsymbol{C}$ contains $\bar{p}$ the function $s(p)$ is multi-valued analytic and bounded over

$$
\omega-\bigcup_{j \in J}\left\{\sigma_{j}(p)=\sigma_{j}(\bar{p})\right\}
$$

where

$$
\sigma_{j}(p) \equiv\left(p_{r(j)}+p_{r^{\prime}(j)}\right)^{2}
$$

A scattering function is said to be multi-valued analytic over a region $\omega^{\prime}$ if and only if the multi-sheeted analytic con- 
tinuation of $s(p)$ along any path that starts at a physical point and remains always over $\omega^{\prime}$ encounters no singularity.

(d) For each $j \in J$ the two-particle threshold value $\sigma_{j} \equiv \sigma_{j}(\bar{p})$ lies below the lowest threshold for the production of three or more particles in the channel $j$.

(e) Extended unitarity holds at $\bar{p}$.

Remark on extended unitarity.

Near a two-particle threshold point $\bar{p}$ the extended unitarity equation says that for some $\varepsilon>0$ the unitarity equations can be extended into the domain

$$
\left\{p \in \mathscr{M}_{r}^{c} ; \bar{\sigma}_{j} \geqq \sigma_{j}(p) \geqq \bar{\sigma}_{j}-\varepsilon, \text { all } j \in J\right\}
$$

with the functions $s(p)$ and $s^{-}(p)$ in this region defined by analytic continuation through $\operatorname{Im} \sigma_{j}>0$, all $j \in J$, and $\operatorname{Im} \sigma_{j}<0$, all $j \in J$, respectively. This extended unitarity property entails that the general $S$-matrix discontinuity formula holds at two-particle threshold points $\bar{p} \in \mathcal{M}_{0}$, where the usual arguments break down. (Coster-Stapp [24].)

Remark about the definition of analyticity.

Two-to-two processes in which the sum of the two initial-particle masses, $\mu_{1}+\mu_{2}$, equals the sum of the two final-particle masses, $\mu_{3}+\mu_{4}$, are important in the following discussions. At a forword-scattering point $\bar{p}$ of such a process the four energy-momentum vectors $\bar{p}_{i},(i=1,2,3,4)$ are all parallel. Hence $\bar{p}$ lies at a singular point of the restricted massshell $\mathcal{M}_{r}$ and the notion of analyticity must be specified.

The restricted mass-shell $\mathscr{M}_{r}$ is a normal analytic set (Hepp [25]). This means that all of the usual definitions of analyticity agree, and are equivalent to the following one:

A function $f$ defined only on the restricted mass shell $\mathscr{M}_{r}$ is analytic at $\bar{p}$ if and only if this function has a local analytic extension $\tilde{f}$ into the imbedding space $\boldsymbol{C}^{4 n}$ such that $\tilde{f}$ is analytic at $\bar{p}$ in the usual sense.

For uniformity we shall adopt this definition also at the regular 
points of $\mathscr{M}_{r}$ even though this definition involves the introduction of trivial off-mass-shell extensions that are not uniquely defined. The massshell results are not affected by these ambiguities.

We assume throughout that each 2-to-2 scattering function $s(p)$, for spinless particles, is a Lorentz scalar, and that its domain of holomorphy is describable in terms of Lorentz scalars. Any 2-to-2 function $f(p)$ with these two properties is analytic at a point $\bar{p} \in \mathscr{M}_{r}$ if and only if it is analytic in the variables $\sigma=\left(p_{1}+p_{2}\right)^{2}$ and $t=\left(p_{1}-p_{3}\right)^{2}$ at the point $\sigma=\bar{\sigma} \equiv\left(\bar{p}_{1}+\bar{p}_{2}\right)^{2}$ and $t=\bar{t} \equiv\left(\bar{p}_{1}-\bar{p}_{3}\right)^{2}$. (Hepp [25].) In place of $t$ one could use $u=\left(p_{1}-p_{4}\right)^{2}$. A more convenient variable is the symmetric combination

$$
\nu=\frac{t-u}{2}+\frac{\left(\mu_{1}^{2}-\mu_{2}^{2}\right)\left(\mu_{3}^{2}-\mu_{4}^{2}\right)}{2 \sigma}
$$

Thus analyticity of the 2-to-2 function $f(p)$ near a two-particle threshold point $\bar{p}$ is equivalent to the analyticity of $f$ in the variables $\sigma$ and $\nu$ at $\sigma=\bar{\sigma}$ and $\nu=\bar{\nu}=0$.

The 2-to-2 case will be considered first. Then the general case will be treated by using the 2 -to- 2 result and the general $S$-matrix discontinuity formula.

\section{Theorem 3. 1.1 .}

Let $s\left(p_{1}, p_{2}, p_{3}, p_{4}\right)$ be the scattering function of a 2-to-2 process, i.e. $S(p)=(2 \pi)^{4} \delta^{4}\left(p_{1}+p_{2}-p_{3}-p_{4}\right) s(p)$. Then in a neighborhood of any two-particle threshold point $\bar{p}$ the scattering function has the form

(3.1.0) $s(p)=a(p)+b(p) \sqrt{\sigma-\bar{\sigma}+i 0}$, where $a(p)$ and $b(p)$ are analytic.

Proof.

First define the 2-to-2 function

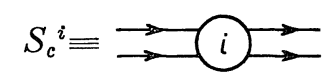

near $\sigma=M_{i}^{2}=\left(m_{l(i)}+m_{l^{\prime}(i)}\right)^{2}$ by the equation:

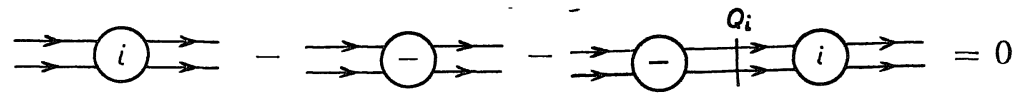


Here $Q_{i}=I-P_{i}$, where $P_{i}$ is the projection operator on states for which the sum of masses of the corresponding particles is equal to, or greater than, $M_{i}^{2}$. Then it is known (Coster-Stapp [24]) that $s^{i}=$ $=S_{c}{ }^{i} /(2 \pi)^{4} \delta\left(p_{1}+p_{2}-p_{3}-p_{4}\right)$ has the minus continuation around $\sigma=M_{i}^{2}$, i.e., it is a boundary value of a holomorphic function attained from the domain $\operatorname{Im} \sigma<0$.

On the other hand, unitarity (or extended unitarity) implies that

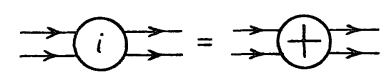

in the region $\sigma<M_{i}^{2}$, below the $i$-th threshold. Thus the function $s^{i}$ in the region $\sigma>M_{i}{ }^{2}$ is the counter-clockwise continuation of the scattering function to under-neath the cut that begins at $\sigma=M_{i}{ }^{2}$. Furthermore, the argument of Coster-Stapp [24] $\S 5$ shows that

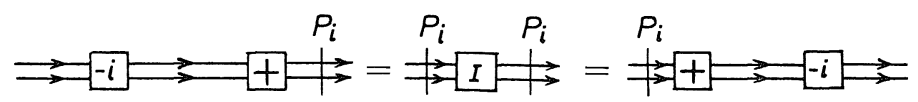

holds, if we define

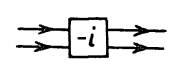

by

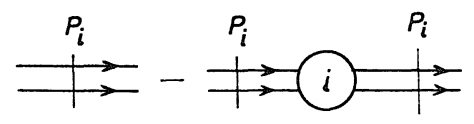

The function $\rightarrow-i \rightarrow$ thus defined coincides (near the threshold point $\sigma=M_{i}^{2}$, which is the point of interest to us) with the $(-\alpha)$-box introduced in $\S 1$, with $i$ in place of $\alpha$.

Now we define $\rho(\sigma)$ by

$$
\frac{\sqrt{\sigma-M_{i}^{2}} \sqrt{\sigma-\left(M_{i}^{-}\right)^{2}}}{2 \pi \sigma}
$$

where $M_{i}^{-}=m_{l(i)}-m_{l^{\prime}(i)}$, and fix $M_{i}^{2}$ at the value $\bar{\sigma}$. Then we define $\rho^{+}$as the boundary value of $\rho(\sigma)$ from the domain $\operatorname{Im} \sigma>0$, and $\rho^{i}$ as that from $\operatorname{Im} \sigma<0$, choosing the branch of $\rho$ so that

$$
\begin{cases}\rho^{+}=-\rho^{i}>0 & \text { for } \sigma>M_{i}^{2} \\ \rho^{+}=\rho^{i} & \text { for } \sigma \leqq M_{i}^{2}\end{cases}
$$


Now following Zimmermann [23] we first expand

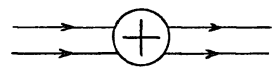

into partial waves, i.e., we expand $s$ into the form

$$
\sum_{l=0}^{\infty}(2 l+1) \Phi_{l}^{i}(\sigma) P_{l}(\cos \theta)
$$

Thus $\Phi_{l}{ }^{+}(\sigma)=\Phi_{l}{ }^{i}(\sigma)$ for $\sigma<M_{i}^{2}$. then

If we define $\Phi_{l}^{+i r}$ by $\Phi_{l}^{+}\left(1+\frac{1}{2} \rho^{+} \Phi_{l}\right)^{-1}$ and $\Phi_{l}^{-i r}$ by $\left(1+\frac{1}{2} \Phi_{l}^{i} \rho^{i}\right)^{-1} \Phi_{l}^{i}$,

$$
\begin{aligned}
\Phi_{l}{ }^{+i r}-\Phi_{l}{ }^{-i r}= & \left(1+\frac{1}{2} \Phi_{l}{ }^{i} \rho^{i}\right)^{-1} \times \\
& \times\left\{\Phi_{l}^{+}-\Phi_{l}{ }^{i}-\frac{1}{2} \Phi_{l}{ }^{i}\left(\rho^{+}-\rho^{i}\right) \Phi_{l}+\right\}\left(1+\frac{1}{2} \rho^{+} \Phi_{l}^{+}\right)^{-1} .
\end{aligned}
$$

The quantity in the bracket vanishes by virtue of (3.1.2).

This implies that $\Phi_{l}^{+i r}\left(=\Phi_{l}^{-i r}\right)$ is actually an analytic function $\Phi_{l}{ }^{i r}(\sigma)$ near $\sigma=M_{i}{ }^{2}$. Thus the solution

$$
\Phi_{l}=\left(1-\frac{1}{2} \Phi_{l}^{i r} \rho^{+}\right)^{-1} \Phi_{l}{ }^{i r}
$$

exhibits the square-root singularity in each partial wave. The boundedness condition (c) ensures that the domain $\omega$ can be made small enough so that all denominators appearing in the above arguments are nonzero.

To obtain the analogous square-root property of the scattering function itself one must examine the domain of convergence of the partial wave series. The arguments of Zimmermann then show that if the domain $\omega$ has the form

$$
\omega=\left\{p ;|\sigma-\bar{\sigma}|<\varepsilon_{1},|\nu|<\varepsilon_{2}\right\}
$$

then the function

$$
\tilde{s}(\delta ; \cos \theta) \equiv s\left(\delta^{2} ; \cos \theta\right)
$$

is holomorphic in

$$
\left\{(\delta, \cos \theta) ;|\delta|<\varepsilon_{1}{ }^{1 / 2}, \cos \theta \in E(\sigma)\right\},
$$

where $E(\sigma)$ is the largest open ellipse with foci at +1 and -1 that 
fits into the circle in the $\cos \theta$ plane defined by

$$
|\nu(\sigma, \cos \theta)|=\left|2 \pi^{2} \sigma \rho_{12}(\sigma) \rho_{34}(\sigma) \cos \theta\right|<\varepsilon_{2},
$$

where $\rho_{12}(\sigma)$ and $\rho_{34}(\sigma)$ are the phase space functions associated with the pairs of mass $\left(\mu_{1}, \mu_{2}\right)$ and $\left(\mu_{3}, \mu_{4}\right)$, respectively.

Now the equation

$$
\begin{aligned}
\tilde{s}(\delta, \nu) & \equiv \tilde{s}\left(\delta ; \cos \theta\left(\nu, \delta^{2}\right)\right) \\
& =\sum_{l=1}^{\infty}(2 l+1) \Phi_{l}(\delta) P_{l}\left(\frac{\nu}{2 \pi^{2} \sigma \rho_{12}(\sigma) \rho_{34}(\sigma)}\right)
\end{aligned}
$$

defines $\tilde{s}(\delta, \nu)$ for all $0<\delta<\varepsilon_{1}{ }^{1 / 2}$ and all $\nu$ in the ellipse $E_{\nu}(\sigma)$ that is the $\nu$-space image of $E(\sigma)$ :

$$
E_{\nu}(\sigma) \equiv\{\nu ; \cos \theta(\nu, \sigma) \in E(\sigma)\} .
$$

This ellipse approaches the disk

$$
\left\{\nu ;|\nu|<\varepsilon_{2}\right\}
$$

as $\sigma$ goes to $\bar{\sigma}$. For $0<|\sigma-\bar{\sigma}|<\varepsilon_{1}$ the expansion of $\tilde{s}(\delta, \nu)$ as a power series in $\nu$ converges for all $|\nu|<\varepsilon_{2}$. since the ellipse $E_{\nu}(\sigma)$ contains points that approach the circle $|\nu|=\varepsilon_{2}$. Thus $\tilde{s}(\delta, \nu)$ is holomorphic in

$$
\widetilde{\omega}=\left\{(\delta, \nu) ;|\delta|<\varepsilon_{1}{ }^{1 / 2},|\nu|<\varepsilon_{2}\right\},
$$

except for a possible isolated singularity at $\delta=0$. The boundedness condition (c) then implies that $\tilde{s}(\delta, \nu)$ is holomorphic in $\widetilde{\omega}$. Thus $s\left(\delta^{2}, \nu\right)$ $=s(\sigma, \nu)$ has the square-root form demanded by the theorem. The fact that $s(\sigma, \nu)$ is defined at real points by the limit from $\operatorname{Im} \sigma>0$ is part of the extended unitarity property.

Q.E.D.

For our purpose, the following version of Theorem 3.1.1 is more convenient.

\section{Theorem 3.1.2.}

There exists an operator

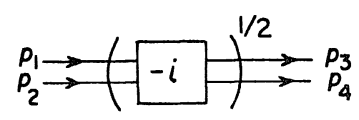


which satisfies the following conditions:
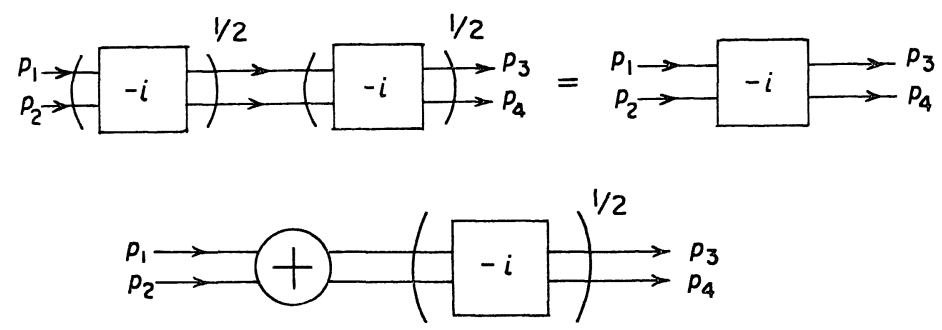

can be written as the product of $Y(\sigma-\bar{\sigma})$ with a function that is analytic in $\left\{p \in \mathscr{M}_{r}{ }^{c} ;|\sigma(p)-\bar{\sigma}|<\varepsilon,|\nu(p)|<\varepsilon\right\}$ for some $\varepsilon>0$.

Proof.

We shall again use the partial wave expansions. Using the same notations as in the proof of the previous theorem, we rewrite the unitarity relation $S S^{\dagger}=I$ in the form

$$
\left(\left(\rho^{+}\right)^{-1}+\Phi_{l}^{+}\right) \rho^{+}\left(\left(\rho^{+}\right)^{-1}-\Phi_{l}^{-}\right)=\left(\rho^{+}\right)^{-1}
$$

if $\sigma$ is near $M_{i}^{2}$ and $\sigma>M_{i}^{2}$.

Define the partial-wave amplitude of the square-root operator by

$$
h_{l}=\left(\rho^{+}\right)^{-1 / 2}\left(\left(\rho^{+}\right)^{-1}-\Phi_{l}^{-}\right)^{1 / 2} \text {. }
$$

Then we find

$$
h_{l} \rho^{+} h_{l}=\left(\left(\rho^{+}\right)^{-1}-\sqrt{-1} \Phi_{l}^{+}\right),
$$

which is the partial-wave form of (3.1.8). On the other hand, the partial wave form of (3.1.9) is, for $\sigma>M_{i}{ }^{2}$,

$$
\begin{gathered}
\left(\frac{\Phi_{l}^{i r}(\sigma)}{1-\frac{1}{2} \rho^{+} \Phi_{l}^{i r}(\sigma)}\right)\left(1-\rho^{+} \frac{\Phi_{l}^{i r}(\sigma)}{1-\frac{1}{2} \rho^{i r} \Phi_{l}^{i r}(\sigma)}\right)^{1 / 2} \\
=\Phi_{l}^{i r}(\sigma)\left(1-\frac{1}{4}\left(\rho^{+}\right)^{2} \Phi_{l}^{i r}(\sigma)^{2}\right)^{-1 / 2}
\end{gathered}
$$

which is nonsingular at $\sigma=\bar{\sigma}$.

Since the convergence of the partial wave series can be verified as before one obtains from the analyticity of (3.1.12) at $\sigma=M_{i}^{2}$ the desired property of (3.1.9) at $\sigma=M_{i}^{2}$.

Q.E.D.

We now generalize Theorem 3.1.1 to many-particle amplitudes. 


\section{Theorem 3.1.3.}

In a neighborhood of a two-particle threshold point $p_{0}$ the scattering amplitude $s(p)$ is simply a product of normal-threshold factors $\left.\left(\left(p_{i}+p_{j}\right)^{2}-\left(\mu_{i}+\mu_{j}\right)^{2}+i 0\right)^{1 / 2} \phi_{i j}+\psi_{i j}\right)$, where $\phi_{i j}$ and $\phi_{i j}$ are analytic.

Remark.

This result validates Sato's conjecture in the neighborhood of any two-particle threshold point, in the sense that it shows that $s(p)$ satisfies a holonomic system. In fact, choosing the various variable $\sigma_{j}$ as independent variables, each normal-threshold factor is clearly a solution of a holonomic system of (micro-) differential equations in these variables (with order -1). Hence as a product of these functions, the function $s(p)$ is also a solution of a holonomic system. However, the characteristic variety is not given by the Landau equations (0.1) at $\mathscr{M}_{0}$-points. On the other hand, Sato makes his conjecture for the off-shell quantity.

Proof of Theorem 3.1.3.

First consider the case where only two external lines are parallel and there are no (communicating) pairs of particles with a smaller sum of masses. The discontinuity formula then asserts that

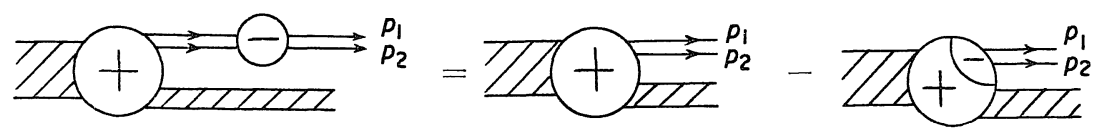

Here

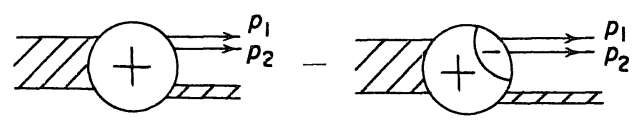

denotes the discontinuity of the scattering amplitude in the $\left(p_{1}, p_{2}\right)$-channel.

Since

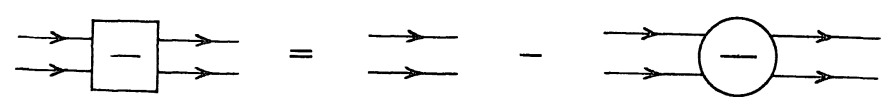

holds, by the definition of box diagram, (3.1.13) can be written 


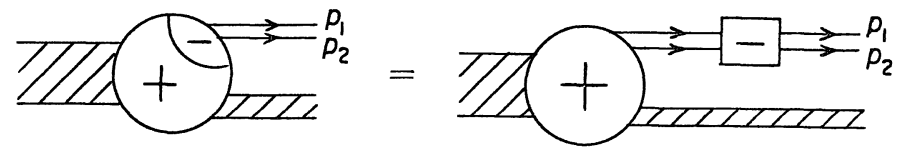

Now we analytically continue both sides of (3.1.14) around the threshold surface $\sigma \equiv\left(p_{1}+p_{2}\right)^{2}=\left(\mu_{1}+\mu_{2}\right)^{2}$ in the $\sigma$-plane counter-clockwise starting from $\left(\mu_{1}+\mu_{2}\right)^{2}+\varepsilon(\varepsilon>0)$. Then, by the definition of

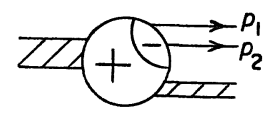

the function

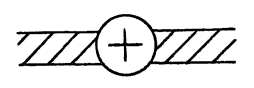

becomes

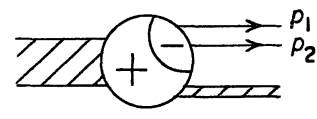

On the other hand, by the two-sheeted structure of

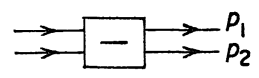

obtained earlier this function (with the two-particle phase-space factor included) becomes

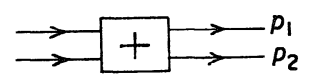

after this analytic continuation. Therefore the right-hand side of (3.1.14) takes the form

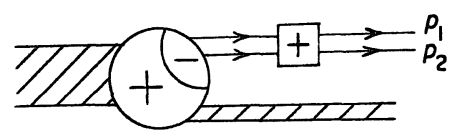

On the other hand, post-multiplying (3.1.14) by

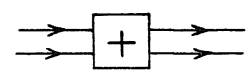

we get 


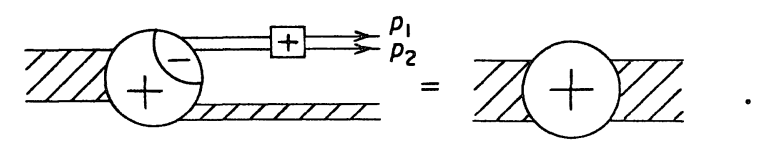

Therefore the left-hand side of (3.1.14) changes into

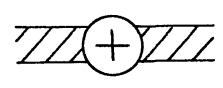

after the analytic continuation around $\sigma=\left(\mu_{1}+\mu_{2}\right)^{2}$. This proves that

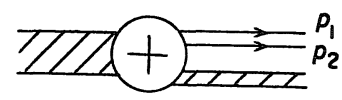

has a two-sheeted structure in the $\sigma$-variable. Then, by virtue of the boundedness condition (c), this function has the form

$$
\phi_{12}(p) \sqrt{\sigma-M_{i}^{2}+i 0}+\psi_{12}(p),
$$

where $\phi_{12}$ and $\phi_{12}$ are analytic.

The above argument covers only the leading two-particle threshold in any channel. However, by using the $i$-box formula (3.1.2) and replacing everywhere the minus box

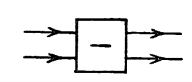

by the minus- $i$ box

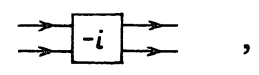

one immediately obtains the generalization to the nonleading case.

Before completing the proof, by extending the argument to the general case where several pairs of lines are relevant, we state and prove two related theorems.

Theorem 3.1.4.

Let $\bar{p}$ be a two-particle threshold point of a function

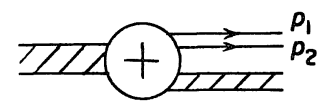

having only one pair of parallel vectors at $\bar{p}$, namely $\bar{p}_{1}$ and $\bar{p}_{2}$. Then 


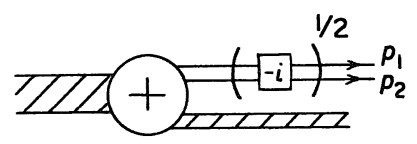

is the product of $Y(\sigma-\bar{\sigma})$ with a function that is analytic at $\sigma=M_{i}$.

Proof.

The proven part of Theorem 3.1.3 claims that

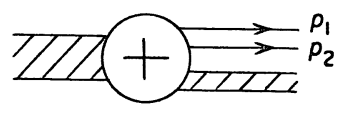

has the form

$$
A(p)+B(p) \rho^{+}
$$

with $A(p)$ and $B(p)$ analytic near $\sigma=M_{i}{ }^{2}$. By Theorem 3.1.1 the function

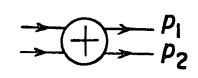

has the form $a(p)+b(p) \rho^{+}$with $a(p)$ and $b(p)$ analytic near $\sigma=M_{i}^{2}$.

On the other hand, the discontinuity formula shows that

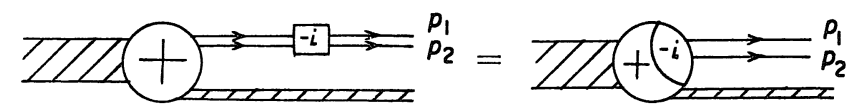

Therefore, using again the partial wave expansion, we find that

$$
\left(A_{l}{ }^{m}+B_{l}{ }^{m} \rho^{+}\right)\left(r_{l}+s_{l} \rho^{+}\right)=\left(A_{l}{ }^{m}-B_{l}{ }^{m} \rho^{+}\right)
$$

holds for any $(l, m)$. Here $r_{l}+s_{l} \rho^{+}$are the coefficients of the partial wave expansion of $\rho^{+}$times

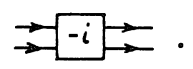

From (3.1.2) we obtain by the same reasoning that

$$
\left(a_{l}+b_{l} \rho^{+}\right)\left(r_{l}+s_{l} \rho^{+}\right)=\left(a_{l}-b_{l} \rho^{+}\right) .
$$

Combining (3.1.16) and (3.1.17) we obtain

$$
A_{l}{ }^{m}+B_{l}{ }^{m} \rho^{+}=c\left(a_{l}+b_{l} \rho^{+}\right)
$$


for any $(l, m)$, where $c(\sigma)$ is analytic in $\sigma$, apart from isolated singularities, since $A_{l}{ }^{m}(\sigma), B_{l}{ }^{m}(\sigma), a_{l}(\sigma)$ and $b_{l}(\sigma)$ are all analytic. ${ }^{*}$ Then the result from Theorem 3.1.2 that

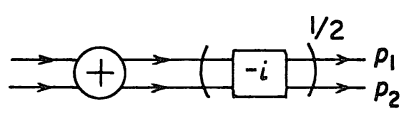

is the product of $Y(\sigma-\bar{\sigma})$ with an analytic function implies the same for

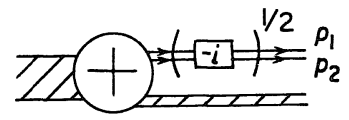

at the partial-wave level, apart from poles. But no partial wave pole can be introduced by multiplication by

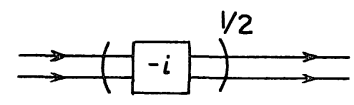

provided $\varepsilon_{1}$ has been chosen small enough so that this factor has no poles in $\omega$. This result at the partial wave level is carried to the level of $s(\sigma, \nu)$ by the same argument as before. $\quad$ Q.E.D.

\section{Theorem 3.1.5.}

Let $\bar{p}$ be a two-particle threshold point. Let

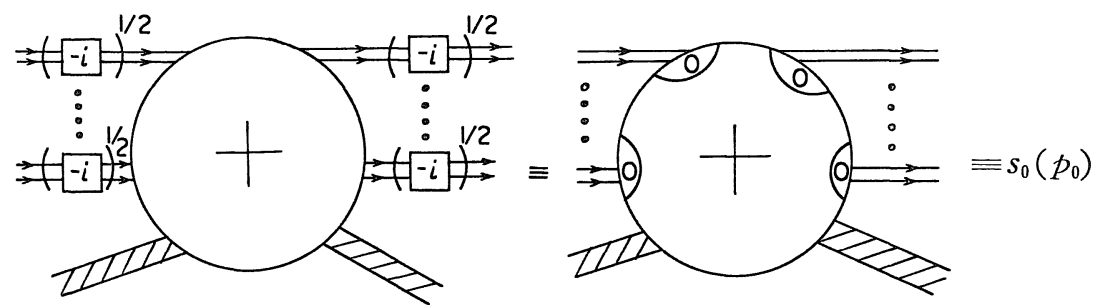

have a factor

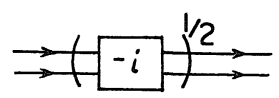

on each pair of lines $j \in J$. Then the corresponding function $s_{0}(p)$ is

* The trivial no-scattering case $a_{l}(\sigma)=b_{l}(\sigma)$, all $\sigma$, is easily treated by a separate argument. 
the product of $\prod_{j \in J} Y\left(\sigma_{j}-\bar{\sigma}_{j}\right)$ with a function that is analytic in a neighborhood of $\stackrel{j \in}{\bar{p}}$.

\section{Proof.}

Consider first the case in which there are precisely two pairs of parallel vectors at $\bar{p}$. Let the two corresponding variables be $\sigma_{1}$ and $\sigma_{2}$, and let $z_{1}=\sigma_{1}-\sigma_{1}(\bar{p})$, and $z_{2}=\sigma_{2}-\sigma_{2}(\bar{p})$. Let the domain $\omega$ be

$$
\omega=\left\{\left|z_{1}\right|<\varepsilon_{1},\left|z_{2}\right|<\varepsilon_{2}, z^{\prime} \in \omega^{\prime}\right\}
$$

where $z^{\prime} \equiv\left(z_{3}, \cdots, z_{4 n-4}\right)$, and the $\varepsilon_{i}$ 's are smaller than the corresponding $\varepsilon_{i}$ 's for the two 2 -to- 2 processes. Then the argument of the previous theorem, applied first to the variable $\sigma_{1}$ shows that the domain of holomorphy of $f(p)$ contains the set

$$
A_{1}=\left\{\left|z_{1}\right|<\varepsilon_{1}\right\} \times\left\{0<x_{2}<\varepsilon_{2}, y_{2}=0\right\} \times\left\{z^{\prime}=z^{\prime}(\bar{p}) \equiv \bar{z}^{\prime}\right\}
$$

The same argument applied to the variable $\sigma_{2}$ shows that the domain of holomorphy contains the set

$$
A_{2}=\left\{0<x_{1}<\varepsilon_{1}, y_{1}=0\right\} \times\left\{\left|z_{2}\right|<\varepsilon_{2}\right\} \times\left\{z^{\prime}=\bar{z}^{\prime}\right\}
$$

But then Bremermann's continuity theorem (See Bros-Epstein-Glaser [26]) implies that the point $\bar{p}$ belongs to the domain of holomorphy. To see this one can consider the disc

$$
D(t)=\left\{\left|z_{1}\right|<\varepsilon_{1}\right\} \times\left\{x_{2}=t, y_{2}=0\right\} \times\left\{z^{\prime}=\bar{z}^{\prime}\right\}
$$

This lies in $A_{1}$ for $0<t<\varepsilon_{2}$. And $D(1)$ contains the point

$$
\left\{z_{2}=\varepsilon_{1} / 2\right\} \times\left\{z_{2}=0\right\} \times\left\{z^{\prime}=\bar{z}^{\prime}\right\}
$$

that lies in $A_{2}$. But then by Bremermann's theorem the disc $D(1)$ lies in the domain of holomorphy of $s_{0}(p)$. But the disc $D(1)$ contains the point

$$
\bar{p}=\left(0,0, \bar{z}^{\prime}\right) .
$$

This argument is easily extended to cover the general case.

Q.E.D.

The usefulness of these results arises from the fact that the part of $T\left(D^{+}\right)$that relates to a two-particle threshold singularity has the form 


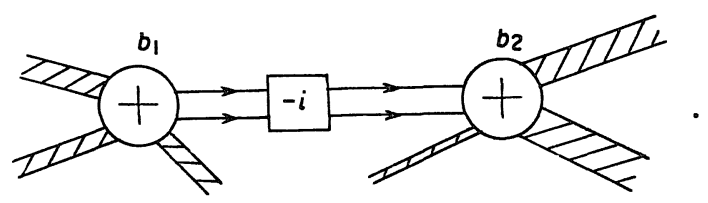

Thus if one breaks the $(-i)$-box into the two square-root factors and multiplies one factor into each of the two plus bubbles then the square root singularities in both of the bubbles are removed. Thus this part of the integral can be replaced simply by the phase space integral

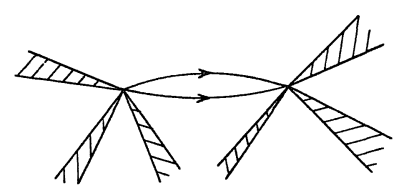

multiplied by some analytic functions. This fact is used in the later arguments.

Completion of proof of Theorem 3.1.3.

From the identities (3.1.5) and (3.1.8) one obtains

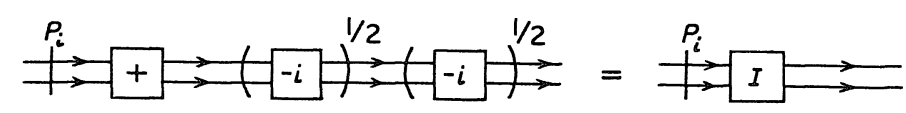

and

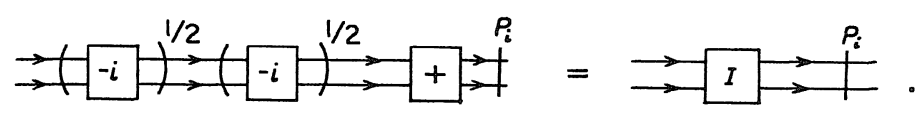

Thus we define

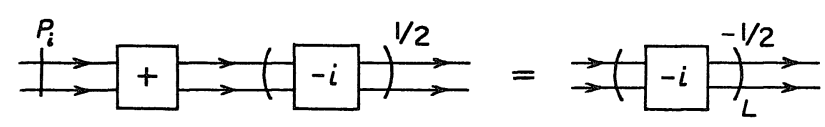

and

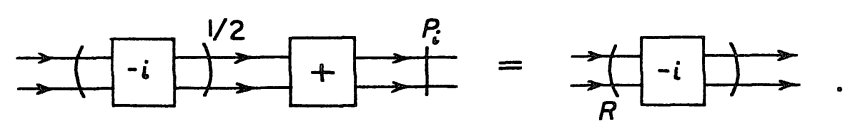

Applying these operators to $s_{0}(p)$ of Theorem 3.1.5 we find that the 
singularities of $s(p)$ near $\bar{p}$ are contained in a product of separate factors, one for each $j \in J$.

Q.E.D.

\section{$\S 3.2$. Study of holonomic structures near a point where a single Landau diagram $\mathbb{D}^{+}$with double lines is relevant}

Making use of Theorem 3.1.5 we can generalize Theorem 2.1.1 to the case where some of the factors in the integrand of the discontinuity function $T\left(D^{+}\right)$are evaluated at two-particle threshold points. First we need the following definition.

\section{Definition 3.2. 1 .}

Let $D$ be a connected diagram that has at most double internal lines i.e., at most two lines connect any pair of vertices of $D$. Then a point $p \in L\left(D^{+}\right)$is said to be elementary with respect to $D^{+}$if and only if the following condition is satisfied:

For each vertex $b$ of $D^{+}$the vector $p^{b}$ defined in (2.1.0) satisfies one of the following two conditions:

(a) $p^{b} \notin L^{+}$(i.e., $p^{b}$ lies at a regular point of the scattering function $s^{b}$ occurring in $\left.T\left(D^{+}\right)\right)$.

(b) $p^{b}$ is a two-particle threshold point of $s^{b}$ such that the pairs $j \in J$ of parallel vectors of $\left(p_{1}^{b}, \cdots, p_{n_{b}}^{b}\right)$ correspond exactly to the pairs of double internal lines of $D$ that touch $b$, and each corresponding two-particle threshold variable $\sigma_{j}^{b}$ lies below the lowest threshold for the production of three or more particles in its associated channel $j$.

The set of points $p \in L\left(D^{+}\right)$that are elementary with respect to $D^{+}$ is denoted by $L_{e}\left(D^{+}\right)$.

\section{Theorem 3.2.1.}

Let $p_{0}$ be a point in $\mathscr{M}-\mathscr{M}_{0}$. Assume that

(i) There is a unique $D$ such that $p_{0} \in L_{0}\left(D^{+}\right)-\mathscr{M}_{0}$.

(ii) This $D$ is connected and has at most double internal lines.

(iii) $p_{0} \in L_{e}\left(D^{+}\right)$.

Then the $S$-matrix $S(p)$ satisfies a simple holonomic system $\mathfrak{M}$ of micro-differential equations on $\sqrt{-1} S^{*} W$ in a neighborhood $W$ of $p_{0}$ 
in $M$. The characteristic variety of $\mathfrak{M}$ is given by the (complexified) Landau equations and the order of $\mathfrak{M}$ is given by $\alpha(D)$.

Proof.

Under the assumptions of the theorem, the microlocal discontinuity formula tells us that we can express microlocally the $S$-matrix $S(p)$ or, equivalently, $T\left(D^{+}\right)$by the bubble diagram function $F^{B}(p)$ with $B$ given by first replacing each vertex of $D$ by the corresponding connected part of the $S$-matrix $S_{j}(p, k)$, keeping simple internal lines of $D$ as they are, and finally replacing each pair of double internal lines by a minus- $\alpha$ box.

\section{Example.}
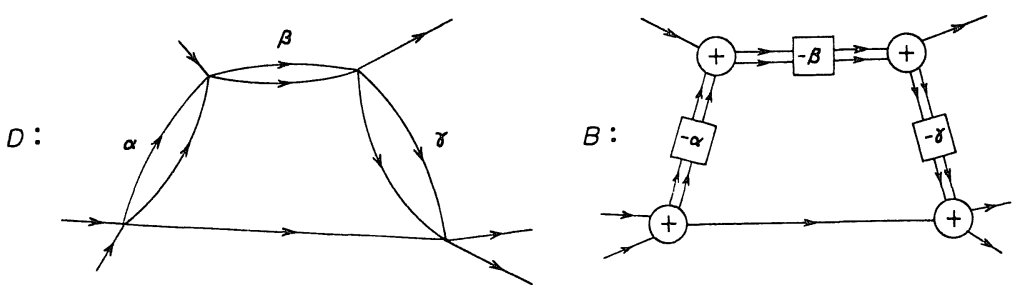

An example of $D$ and the associated bubble diagram $B$

Then in view of Theorem 3.1.5, the resulting function has the form

$$
\int \prod_{j=1}^{n^{\prime}} f_{j}(p, k) \delta^{4}\left(\sum_{r=1}^{n}[j: r] p_{r}+\sum_{l=1}^{N}[j: l] k_{l}\right) \prod_{l=1}^{N} \delta^{+}\left(k_{l}{ }^{2}-m_{l}{ }^{2}\right) \prod_{l=1}^{N} d^{4} k_{l}
$$

with some analytic functions $f_{j}(p, k)$ determined by the scattering amplitude inserted into $V_{j}$ of $D$. This integral is of the same form as the integral studied in Theorem 2.1.1. Hence we can apply the argument given there to conclude that the $S$-matrix $S(p)$ satisfies (micro-) locally a simple holonomic system of micro-differential equations whose characteristic variety is given by the Landau variety associated with $D$ and whose order is $\alpha(D)$.

Q.E.D.

Since Corollary 1 of Theorem 2.1.1 depends only on the existence of a simple holonomic system and the codimension 1 character of the 
Landau "surface", we have also the following result:

\section{Corollary.}

Under the assumptions of Theorem 3.2.1, the S-matrix $S(p)$ has the following form:

$$
\left\{\begin{array}{l}
\delta^{4}\left(\sum_{j, r}[j: r] p_{r}\right)\left(h_{1}(p)(\phi(p)+i 0)^{-\alpha+3 / 2}+h_{2}(p)\right) \\
\quad \text { if }-\alpha+\frac{3}{2} \equiv-\alpha(D)+\frac{3}{2} \text { is neither a positive } \\
\text { integer nor zero } \\
\text { or } \quad \delta^{4}\left(\sum_{j, r}[j: r] p_{r}\right)\left(h_{1}(p) \phi(p)^{-\alpha+3 / 2} \log (\phi(p)+i 0)+h_{2}(p)\right) \\
\quad \text { if }-\alpha+\frac{3}{2} \text { is a positive integer or zero. }
\end{array}\right.
$$

In view of the microlocal form of the discontinuity formula, it is easy to obtain a result corresponding to Theorem 2.1.3 even when $D^{+}$ has some pairs of double lines, by the same reasoning given above. We leave the details to the reader.

\section{$\S 3.3$. Verification of Sato's conjecture at the points where several Landau diagrams with double internal lines are relevant}

In this section we generalize Theorem 2.2.1 to the case where the relevant diagrams allow pairs of double lines.

\section{Theorem 3.3. 1 .}

Let $\left(p_{0} ; \sqrt{-1}, u_{0}\right)$ be a point in $\sqrt{-1} S^{*}\left(\mathscr{M}-\mathscr{M}_{0}\right)$. Let $D$ be a connected Landau diagram with at most double internal lines. Suppose the following four conditions are satisfied:

(3.3.1) Same as (2.2.1)

(3.3.2) For each vertex $b$ of $D$ the vector $p^{b}$ defined in (2.1.0) satisfies one of the following three conditions:

(a) $p^{b} \notin L^{+}$

(b) The following three conditions are satisfied:

(i) there is a unique $D_{b}$ such that $p^{b}$ lies on $L_{0}\left(D_{b}\right)-\mathscr{M}_{0}$. 
(ii) this $D_{b}$ is connected and has at most double internal lines.

(iii) $p^{b} \in L_{e}\left(D_{b}{ }^{+}\right)$

(c) Same as condition (b) of Definition 3.2.1.

\section{(3.3.3) Same as (2.2.3)}

(3.3.4) Same as (2.2.4)

Then the $S$-matrix $S(p)$ satisfies a holonomic system $\mathfrak{M}$ of microdifferential equations in a neighborhood of $\left(p_{0} ; \sqrt{-1} u_{0}\right)$. Furthermore, the characteristic variety of $\mathfrak{M}$ is given by the (complexified) Landau equations.

Proof.

Under the assumptions of the theorem, $T\left(D^{+}\right)$has the following form:

$$
\int \prod_{b} s^{b}(p, k) \delta^{4}\left(\sum_{r}[b: r] p_{r}+\sum_{l}[b: l] k_{l}\right) \prod_{l} \delta^{+}\left(k_{l}{ }^{2}-m_{l}{ }^{2}\right) d^{4} k_{l},
$$

where $s^{b}(p, k)$ has the form

$$
A^{b}(p, k) \Phi_{\lambda}\left(\phi^{b}(p, k)+i 0\right)+B^{b}(p, k)
$$

with analytic functions $A^{b}$ and $B^{b}$. Here $A^{b}$ is zero if $S^{b}$ is evaluated outside its Landau varieties or at the two-particle threshold points. As in the proof of Theorem 2.2.1, $\lambda$ is given by $\alpha\left(D_{B}\right)-\frac{5}{2}$ if $A^{b} \neq 0$.

Since (3.3.6) has the same form as (2.2.7), the argument in $\S 2.2$ applies immediately to this case, and establishes the required results for $F^{B}(p)$.

On the other hand, the microlocal form of the discontinuity formula claims that the $S$-matrix $S(p)$ is equal to $T\left(D^{+}\right)$in a neighborhood of $\left(p_{0} ; \sqrt{-1} u_{0}\right)$. Hence we obtain the required results for the $S$-matrix.

$$
\text { Q.E.D. }
$$

\section{Remark.}

In Theorem 2.2.1 and Theorem 3.3.1, we considered exclusively points where the corresponding $S^{b}$ is evaluated either at two-particle threshold points or at $L_{e}\left(D_{b}\right)$ or at its regular points. However, it will 
be obvious that more complicated singularities can be now considered by inserting the results obtained in examples at the end of $\S 2.2$.

One should be able by iteration to obtain the holonomic system of micro-differential equations that the $S$-matrix satisfies as long as the number of relevant (complexified) Landau varieties are finite, and the point in question is not a $u=0$ point for any of the relevant Landau diagrams.

\section{§4. Structure of the $S$-matrix at points where the Landau surface associated with a triangle diagram meets the surface associated with the self-energy diagram}

The analysis in $\S 2$ and $\S 3$ does not cover the case described by the title of this section. For the analysis of this case we shall impose a condition on the singularity structure of the scattering function in a complex domain. This condition, which is condition (c) below, is analogous to condition (c) of Definition 3.1.1. It does not follow (by local analysis) from the microanalyticity, unitarity, and Lorentz invariance properties of the $S$-matrix.

Consider a scattering function $s(p)$ and a value $\bar{p}$ of its argument. Suppose the following conditions are satisfied:

(a) Let $\left\{D_{j} ; j \in J\right\}$ be the set of unsigned diagrams such that $\bar{p} \in \bar{L}_{0}\left(D_{j}{ }^{+}\right)$. Then this set consists of the following two diagrams :
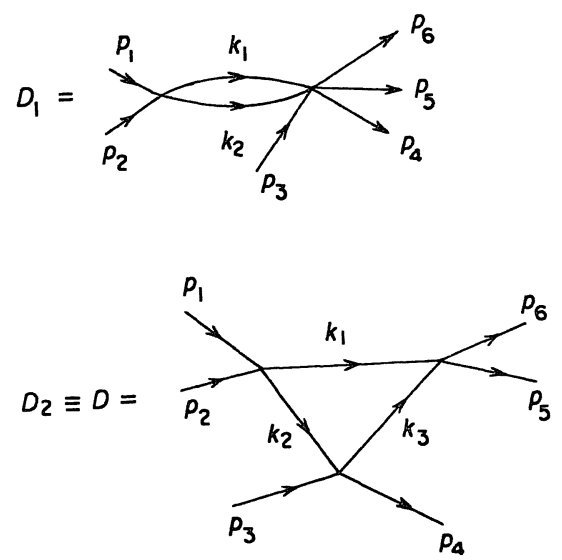
(b) $\mu_{1}+\mu_{2}<m_{1}+m_{2}$ where $p_{r}^{2}=\mu_{r}^{2}$ and $k_{l}{ }^{2}=m_{l}{ }^{2}$.

(c) For every sufficiently small complex neighborhood $\widetilde{\omega}$ in the space of Lorentz scalars such that its inverse image $\omega$ in $\mathcal{M}_{r}{ }^{\boldsymbol{C}}$ contains $\bar{p}$ the scattering function $s(p)$ is multi-valued analytic over $\omega-\left(\left\{\phi_{1}(p)=0\right\} \cup\left\{\phi_{2}(p)=0\right\}\right)$, where $\phi_{1}(p)=0$ defines $L^{\boldsymbol{C}}\left(D_{1}\right)$ near $\bar{p}$ and $\phi(p)=0$ defines $L^{\boldsymbol{C}}\left(D_{2}\right)$ near $\bar{p}$.

(d) The variable $\sigma=\left(\bar{p}_{1}+\bar{p}_{2}\right)^{2}$ lies below the lowest threshold for the production of three or more particles in the channel corresponding to particles 1 and 2 .

In this case we shall show that the scattering function $s(p)$ has, near $\bar{p}$, the form described in (4.2) below. To describe this form we introduce a local coordinate system in a neighborhood of $\bar{p}$ so that $\bar{p}$ is the image of the origin $x=0$ and $\pi\left(\mathcal{L}^{\mathbb{C}}\left(D^{+}\right)\right)=L^{\mathbb{C}}\left(D^{+}\right)$is given by $\left\{x ; x_{1}=x_{2}^{2}\right\}$ and $\pi\left(\mathcal{L}^{c}\left(D_{1}^{+}\right)\right)=L^{C}\left(D_{1}^{+}\right)$is given by $\left\{x ; x_{1}=0\right\}$. We may assume further that $\pi\left(\mathcal{L}\left(D^{+}\right)\right) \subset\left\{x\right.$ real and $\left.x_{1}=x_{2}, x_{2} \leqq 0\right\}$. Then in a sufficiently small neighborhood of $\bar{p}$

$$
\begin{aligned}
s(p)= & \phi_{1}(x) \sqrt{x_{1}+i 0} \log \left(\sqrt{x_{1}+i 0}+x_{2}\right)+ \\
& +\phi_{2}(x) \log \left(\sqrt{x_{1}+i 0}+x_{2}\right)+\phi_{3}(x) \sqrt{x_{1}+i 0}+ \\
& +\phi_{4}(x)
\end{aligned}
$$

with analytic functions $\phi_{j}(x) \quad(j=1, \cdots, 4)$.

We now show how this expression can be obtained:

First define the function

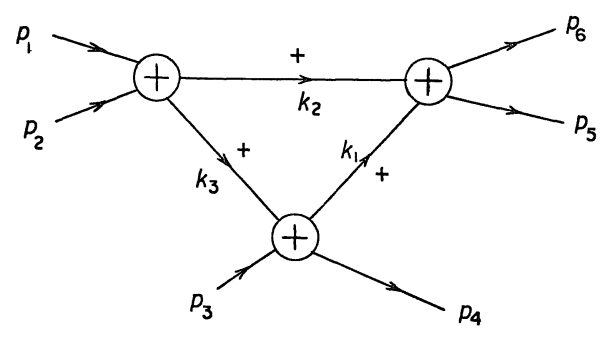

by the integral

$$
F(p) \equiv F_{D^{+}}(p)=\frac{1}{(2 \pi)^{12}} \int S\left(p_{1}, p_{2}, k_{2}, k_{3}\right) S\left(k_{3}, p_{3}, k_{1}, p_{4}\right) S\left(k_{1}, k_{2}, p_{5}, p_{6}\right) \times
$$




$$
\times \prod_{l=1}^{3} \frac{Y\left(k_{l, 0}\right) Y\left(k_{l}{ }^{2}-m_{l}{ }^{2}+\varepsilon_{l}{ }^{\prime}\right) Y\left(m_{l}{ }^{2}+\varepsilon_{l}-k_{l}{ }^{2}\right)}{k_{l}{ }^{2}-m_{l}{ }^{2}+i 0} \prod_{l=1}^{3} d^{4} k_{l} .
$$

That is, $F_{D^{+}}(p)$ is constructed by rules that are the same as those used to construct bubble diagram functions except that a signed internal line $L_{l}$ is replaced by a factor

$$
\frac{i Y\left(k_{l, 0}\right) Y\left(k_{l}{ }^{2}-m_{l}{ }^{2}+\varepsilon_{l}{ }^{\prime}\right) Y\left(m_{l}{ }^{2}+\varepsilon_{l}-k_{l}{ }^{2}\right)}{k_{l}{ }^{2}-m_{l}{ }^{2}+i 0}
$$

whereas an unsigned internal line $L_{l}$ of a bubble diagram is replaced by

$$
2 \pi \delta\left(k_{l}{ }^{2}-m_{l}{ }^{2}\right) Y\left(k_{l, 0}\right) \text {. }
$$

A slight generalization of the arguments in $\S 2.1$ shows that $F(p)$ near $p_{0}$ is a well-defined hyperfunction, provided the constants $\varepsilon_{l}$ and $\varepsilon_{l}{ }^{\prime}$ are chosen sufficiently small $(l=1,2,3)$. Furthermore, those arguments show that the singularity spectrum of $F(p)$ is confined to $\mathcal{L}\left(D^{+}\right) \cup \mathcal{L}\left(D_{1}^{+}\right)$ near the origin (cf. the proof of Structure Theorem in $\S 0$ ). Therefore $f(p) \equiv F(p) /(2 \pi)^{4} \delta^{4}\left(\sum_{r=1}^{6} \varepsilon_{r} p_{r}\right)$ is a boundary value of a holomorphic function $\tilde{f}(p)$ that is defined and holomorphic in $\left\{x ; \operatorname{Im} x_{1}>0\right\} \cap\left\{x ; \operatorname{Im}\left(x_{1}-x_{2}{ }^{2}\right)\right.$ $>0\} \cap\left\{x:\left|x_{1}\right|^{2}+\left|x_{2}\right|^{2}<\varepsilon\right\}$ for some positive $\varepsilon$. Moreover, the result of $\S 3.2$ entails that $S\left(p_{1}, p_{2}, k_{2}, k_{3}\right)$ has the form

$$
\delta^{4}\left(p_{1}+p_{2}-k_{2}-k_{3}\right)\left(\widetilde{\phi}(p, k) \sqrt{\left(k_{2}+k_{3}\right)^{2}-\left(m_{2}+m_{3}\right)^{2}+i 0}+\widetilde{\psi}(p, k)\right)
$$

with $\widetilde{\phi}$ and $\widetilde{\psi}$ analytic functions of $(p, k)$ at the point where they are evaluated. Therefore in a neighborhood of the point $\bar{p}$

$$
\begin{aligned}
F(p)= & \sqrt{\left(p_{1}+p_{2}\right)^{2}-\left(m_{2}+m_{3}\right)^{2}+i 0} \int \delta^{4}\left(p_{1}+p_{2}-k_{2}-k_{3}\right) \times \\
& \times \delta^{4}\left(k_{3}+p_{3}-k_{1}-p_{4}\right) \delta^{4}\left(k_{1}+k_{2}-p_{5}-p_{6}\right) \widetilde{\phi}(p, k) \prod_{j=2}^{3} \psi_{j}(p, k) \times \\
& \times \frac{\prod_{l=1}^{3} i Y\left(k_{l, 0}\right) Y\left(k_{l}{ }^{2}-m_{l}{ }^{2}+\varepsilon_{l}{ }^{\prime}\right) Y\left(m_{l}{ }^{2}+\varepsilon_{l}-k_{l}{ }^{2}\right)}{\prod_{l=1}^{3}\left(k_{l}{ }^{2}-m_{l}{ }^{2}+i 0\right)} \prod_{l=1}^{3} d^{4} k_{l}+ \\
& +\int \delta^{4}\left(p_{1}+p_{2}-k_{2}-k_{3}\right) \delta^{4}\left(k_{3}+p_{3}-k_{1}-p_{4}\right) \delta^{4}\left(k_{1}+k_{2}-p_{5}-p_{6}\right) \times \\
& \times \widetilde{\psi}(p, k) \prod_{j=2}^{3} \psi_{j}(p, k)
\end{aligned}
$$




$$
\times \frac{\prod_{l=1}^{3} i Y\left(k_{l, 0}\right) Y\left(k_{l}{ }^{2}-m_{l}{ }^{2}+\varepsilon_{l}{ }^{\prime}\right) Y\left(m_{l}{ }^{2}+\varepsilon_{l}-k_{l}{ }^{2}\right)}{\prod_{l=1}^{3}\left(k_{l}{ }^{2}-m_{l}{ }^{2}+i 0\right)} \prod_{l=1}^{3} d^{4} k_{l},
$$

where $\phi_{j}(j=1,2,3)$ are analytic.

By the same argument as in $\S 2.2$, we can conclude that $f(p)$ has the form

$$
\begin{aligned}
f(p)= & \left(a_{1}(x) \sqrt{x_{1}+i 0}+a_{2}(x)\right) \times \\
& \times\left(a_{3}(x) \log \left(\sqrt{x_{1}+i 0}+x_{2}\right)+a_{4}(x) \sqrt{x_{1}+i 0}+a_{5}(x)\right)
\end{aligned}
$$

with the $a_{j}(x), j=1, \cdots, 5$, analytic.

To complete the proof we show that

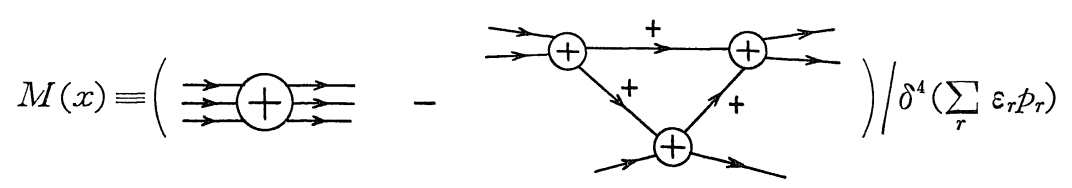

is analytic in $\delta \equiv \sqrt{x_{1}}$ and $x^{\prime} \equiv\left(x_{2}, x^{\prime \prime}\right)=\left(x_{2}, x_{3}, \cdots, x_{14}\right)$.

By our assumptions, the function

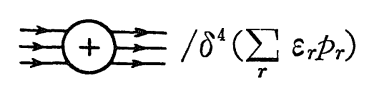

is expressed as a boundary value of a holomorphic function that can be analytically continued outside $\left\{x_{1}=x_{2}^{2}\right\} \cup\left\{x_{1}=0\right\}$. On the other hand, the discontinuity formula implies that $M(x)$ can be analytically continued throughout the intersection of a real neighborhood of the origin with $x_{2} \leqq 0$, except at $\left\{x_{1}=0\right\}$. And the microanalyticity assumption entails that this same property holds also in $\left\{x_{2} \geqq 0\right\}$. Thus the following lemma implies that $M(x)$ is multivalued analytic in a complex neighborhood of the origin except on $\left\{x_{1}=0\right\}$.

\section{Lemma 4. 1.}

Suppose a function $f(z)$ has a domain of holomorphy $\mathscr{D} \subset \mathbb{C}^{n}$ that contains all real points in the region

$$
\omega^{\prime}=\omega-\left\{z ; z_{1}=0 \text { or } z_{1}=z_{2}^{2}\right\},
$$

where $\omega$ is a closed neighborhood of the form

$$
\omega \equiv\left\{z ;\left|z_{i}\right| \leqq \varepsilon_{i}, \varepsilon_{i}>0, i=1, \cdots, n\right\} .
$$


Suppose $f(z)$ can be analytically continued along any path lying over $\omega^{\prime}$; i.e., $f(z)$ is multivalued analytic over $\omega^{\prime}$. Suppose the original domain of holomorphy $\mathscr{D}$ contains two interior points of $\omega$ of the form

$$
\begin{array}{ll}
x_{1}=a^{2}, & x_{2}=a, \quad y_{1}=y_{2}=0, \quad a>0, \\
x_{1}=a^{2}, \quad x_{2}=-a, \quad y_{1}=y_{2}=0, &
\end{array}
$$

where $x_{i}$ and $y_{i}$ are the real and imaginary parts of $z_{i}$.

Then $f(z)$ is multivalued analytic over

$$
\omega \cap\left\{z: 0<\left|z_{1}\right|<\varepsilon_{2}^{2},\left|z_{i}\right|<\varepsilon_{i}, i=1, \cdots, n\right\}
$$

Proof.

Let $t$ be a real parameter that runs from 0 to $\infty$. Consider the one-parameter family of discs

$$
D(t) \equiv\left\{z_{1}=z_{1}(t),\left|z_{2}\right| \leqq \varepsilon_{2}, \text { all other } z_{1} \text { fixed with }\left|z_{i}\right| \leqq \varepsilon_{i}\right\},
$$

where the function $z_{1}(t)$ defines a continuous path in $z_{1}$ space that starts at $z_{1}(0)=a^{2}$. Then the initial disc $D(0)$ lies in $\mathscr{D}$ because only singularities with $z_{2}= \pm a$ are allowed by (4.4), and these are excluded by (4.5). Since domains of holomorphy are open, all disc $D(t), 0 \leqq t<\eta$, must lie in $\mathscr{D}$ for some sufficiently small $\eta$. Let the path in $z_{1}$ space be any continuous path through $z_{1}=a$ that lies in

$$
0<\left|z_{1}\right|<\varepsilon_{2}{ }^{1 / 2}
$$

Then, by virtue of (4.4), any singularity that appears in the (moving) disc $D(t)$ must appear at an interior point of the disc. This is precluded by the Kontinuitätssatz (Behnke and Sommer [27]. See also Wightman [28]). Thus the part of $\omega$ satisfying (4.7) is free of singularities on all sheets. This gives the required result (4.6).

Q.E.D.

This lemma shows that $M(x)$ is multivalued analytic near the origin outside of $\left\{x_{1}=0\right\}$. Thus the function

$$
\widetilde{M}\left(\delta, x^{\prime}\right) \equiv M\left(\delta^{2}, x^{\prime}\right) \equiv M\left(x_{1}, x^{\prime}\right)
$$

must be analytic near the origin except on $\delta=0$. However, the result (4.3) combined with the result of $\S 3.2$ on the square-root character of the two-particle normal-threshold singularity of the $S$-matrix implies that 
near the origin $\widetilde{M}\left(\delta, x^{\prime}\right)$ is analytic on $\delta=0$ except possibly at $x_{2}=0$. This possible isolated singularity at $\delta=0$ that is allowed only for $x_{2}=0$ is also ruled out by the Kontinuitätssatz. Thus $\widetilde{M}\left(\delta, x^{\prime}\right)$ is analytic at $\delta=x^{\prime}=0$ and $M(x)$ must have the form

$$
M(x)=m_{1}(x)+m_{2}(x) \sqrt{x_{1}+i 0}
$$

with analytic $m_{1}$ and $m_{2}$. Combining this result with (4.3) one obtains the desired (4.2).

Q.E.D.

\section{§ 5. Maximal analyticity and holonomicity}

We have shown in several cases involving only simple diagrams that the holonomicity of the $S$-matrix follows directly from microanalyticity and the general $S$-matrix discontinuity formula. However, for more complicated cases a stronger analyticity requirement was needed. This assumption, when cast into general form, asserts the following:

Let $p=\left(p_{1}, \cdots, p_{n}\right)$ be any point in $\mathcal{M}_{r}$ and let $z(p)=\left(z_{1}(p), \cdots, z_{\lambda}(p)\right)$ be the corresponding point in the space of all the bilinear Lorentz scalars formed from the vectors of $p$. Let $\widetilde{\omega}(p) \equiv\left\{z ;\left|z_{i}-z_{i}(p)\right|^{2}<\varepsilon_{i}, \varepsilon_{i}>0, i=1, \cdots, \lambda\right\}$ be a polycylinder in $z$ space centered at $z(p)$ and let $\omega(p)$ be its (inverse) image in $\mathscr{M}_{r}$. Then for any $p$ in $\mathscr{M}_{r}$ and every sufficiently small $\widetilde{\omega}(p)$ the singularities of the multi-valued analytic continuation of $s(p)$ over $\omega(p)$ are confined to the union of the local complexifications over $\omega(p)$ of the positive- $\alpha$ Landau surfaces that pass through $p$. The local complexification over $\omega(p)$ of a positive- $\alpha$ Landau surface is the set of solutions of the complexified equations that can be reached by a path in the space of the variables $(p, u, k, v, \alpha, \beta)$ that starts at a positive- $\alpha$ solution and remains always over $\omega(p)$.

This analyticity assumption is physically reasonable: if the singularities at real points are confined to the positive- $\alpha$ solutions, then the singularities at nearby points should be confined to nearby solutions.

From a mathematical point of view the question arises whether holonomicity could be derived from a weaker assumption. However, the more important question for physics, at least from the viewpoint of $S$ matrix theory, is not how weak the analyticity assumption can be made without losing holonomicity, but rather how strong the analyticity as- 
sumption can be made without violating the microanalyticity, unitarity or Lorentz invariance properties of the $S$-matrix. For the principle of maximal analyticity (Chew [29], Stapp [30], Gunson [31]) asserts that the $S$-matrix has the minimal singularity structure consistent with these three principles: it instructs us at each stage of our understanding of the analytic structure of the $S$-matrix to demand the minimal singularity structure compatible with that understanding.

As an initial application of this general principle we can demand "local maximal analyticity", which is the property enunciated at the beginning of this section and exemplified in the analyticity properties (c) of $\S 3.1$ and $\S 4.1$. One can also include in local maximal analyticity the condition that $s(p)$ be bounded near $\bar{p}$, provided $\bar{p}$ lies on no $L_{0}\left(D^{+}\right)$ for which $\alpha(D)=5 / 2$ or 2 . Local maximal analyticity, defined in this way, appears to be in no way incompatible with the microanalyticity, unitarity, and Lorentz invariance properties of the $S$-matrix.

The analysis of the preceding section shows that if we assume microanalyticity and the general discontinuity formula then at many points $p \in \mathcal{M}_{r}$ local maximal analyticity entails local holonomicity. Two questions thus arise:

(1) Does local maximal analyticity entail local holonomicity at every point $p \in \mathscr{M}_{r}$ ?

(2) If not, then is local holonomicity at least consistent with local maximal analyticity, and the other $S$-matrix principles and results, including in particular the general discontinuity formula?

If local holonomicity is consistent with these other requirements then it should, according to the general principle of maximal analyticity, be accepted as a general property of the $S$-matrix.

The present work has answered neither of these two questions. We hope it has laid an adequate foundation for their further study.

Local holonomicity at points $p \in \mathcal{M}_{r}$ places a condition on the character of the singularity structure of the $S$-matrix at physical points. It is therefore of interest in its own right. But its greater potential importance lies in its relevance to the deeper questions of whether it is possible to demand global holonomicity of the $S$-matrix, and what the impact of such a condition would be on the allowed form of the $S$-matrix. These deeper questions also remain unanswered. 


\section{References}

[1] Sato, M., Recent development in hyperfunction theory and its application to physics. Lecture Notes in Physics, No. 39, pp. 13-29, Berlin-Heidelberg-New York, Springer, 1975.

[2] Sato, M., T. Kawai, and M. Kashiwara, Microfunctions and pseudo-differential equations. Lecture Notes in Math. No. 287, pp. 265-529, Berlin-Heidelberg-New York, Springer, 1973.

[3] Kawai, T. and H. Stapp, Micro-local study of the $S$-matrix singularity structure. Lecture Notes in Physics, No. 39, pp. 36-48, Berlin-Heidelberg-New York, Springer, 1975.

[4] Pham, F., Singularités des processus de diffusion multiple. Ann. Inst. H. Poincaré, 6A (1967), 89-204.

[5] Stapp, H., Discontinuity formulas for multiparticle amplitudes. Structural Analysis of Collision Amplitudes, pp. 191-273, Amsterdam, North Holland, 1976.

[6] Kashiwara, M., T. Kawai, and H. Stapp, Micro-analytic structure of the $S$-matrix and related functions. The proceeding paper.

[7] Chandler, C. and H. Stapp, Macroscopic causality conditions and properties of scattering amplitudes. J. Math. Phys., 10 (1969), 826-859.

[8] Iagolnitzer, D. and H. Stapp, Macroscopic causality and physical region analyticity in $S$-matrix theory. Commun. math. Phys., 14 (1969), 15-55.

[9] Pham, F., Microanalyticité de la matrice S. Lecture Notes in Math., No. 499, pp. 83-101, Berlin-Heidelberg-New York. Springer, 1975.

[10] Sato, M., Ref. 1.

[11] Kawai, T. and H. Stapp, Ref. 3.

[12] Iagolnitzer, D., Analyticity property of scattering amplitudes: a review of some recent developments. Lecture Notes in Physics, No. 39, pp. 1-21, Berlin-HeidelbergNew York, Springer, 1975.

[13] Stapp, H., Finiteness of the number of positive- $\alpha$ Landau surfaces in bounded portions of the physical region. J. Math. Phys., 8 (1967), 1606-1610.

[14] Stapp, H., Crossing, Hermitian analyticity, and the connection between spin and statistics. J. Math. Phys., 9 (1963), 1548-1592.

[15] Iagolnitzer, D., The structure theorem in $S$-matrix theory. Commun. math. Phys., 41 (1975), 39-53.

[16] Kawai, T. and H. Stapp, Ref. 3.

[17] Bros, J. and D. Iagolnitzer, Tuboïdes et structure analytique des distributions. Séminaires Goulaonic-Lions-Schwartz 1974/1975, exposés 16 and 18, and Iagolnitzer, D. Analytic structure of distributions and essential support theory. Structural Analysis of Collision Amplitudes. Part III. Amsterdam, North Holland, 1976.

[18] Iagolnitzer, D. and H. Stapp, The pole-factorization theorem in $S$-matrix theory (in preparation).

[19] Coster, J. and H. Stapp, Physical-region discontinuity equations. J. Math. Phys., 11 (1970), 2743-2763.

[20] Grauert, H., On Levi's problem and the imbedding of real-analytic manifolds. Ann. Math., 68 (1958), 460-472.

[21] Eden, R., P. Landshoff, D. Olive, and J. Polkinghorne, The Analytic S-matrix. Cambridge, Cambridge Univ. Press, 1966.

[22] Kashiwara, M., T. Kawai, and T. Oshima, Micro-differential equations and the singularity structure of Feynman integrals (in preparation).

[23] Zimmermann, W., Analytic behavior of the scattering amplitude at zero energy. Nuovo Cimento, 21 (1961). 249-273.

[24] Coster, J. and H. Stapp, Physical-region discontinuity equations for many-particle 
scattering amplitude. I. J. Math. Phys.. 10 (1969), 371-396.

[25] Hepp, K., Lorentz invariant analytic $S$-matrix amplitudes. Helv. Phys. Acta, 37 (1964), 55-73.

[26] Bros, J., H. Epstein, and V. Glaser, Some rigorous analyticity properties of the four-point function in momentum space. Nuovo Cimento, 31 (1964), 1265-1302.

[27] Behnke, H. and F. Sommer, Analytische Funktionen mehrer komplexes Veränderlichen. Über die Voraussetzungen des Konintuitätssatzer. Math. Annalen, 121 (1950), 356-378.

[28] Wightman, A., Analytic functions of several complex variables. Dispersion relations and elementary particles, ed. by DeWitt, C. and R. Omnes, pp. 227-315, especially pp. 259-262, New York, John Wiley and Sons, 1960.

[29] Chew, G., S-matrix Theory of Strong Interactions. New York, Benjamin, 1961.

[30] Stapp, H., Derivation of the CPT theorem and the connection between spin and statistics from postulates of the $S$-matrix theory. Phys. Rev., 125 (1962), 2139-2162.

[31] Gunson, J., Unitarity and on-mass-shell analyticity as a basis for $S$-matrix theories. I. and II. J. Math. Phys., 6 (1965), 827-844 and 845-851. First issued as a University of Birmingham preprint (1962).

[32] Nakanishi, N., Graph Theory and Feynman Integrals. New York-London-Paris, Gordon and Breach, 1971. 